\title{
A STUDY ON MARINE FISHERY RESOURCES OF ANDHRA PRADESH: ECOLOGICAL ASPECTS AND MORPHOMETRICS OF COMMON MARINE FISHES OF VISAKHAPATNAM - PROTEIN CONTENT AND BIOACCUMULATION OF HEAVY METALS IN POMFRET FISH SPECIES
}

Bhushanam Jeevan Prasad KAMMARCHEDU * and Jacob Solomon Raju ALURI **

* Andhra University, Department of Environmental Sciences, Visakhapatnam, IN-530003, India, kbjpamith@gmail.com, ORCID: 0000-0003-1634-4791, solomonraju@gmail.com, ORCID: 0000-00020028-2621.

DOI: 10.2478/trser-2021-0016

KEYWORDS: marine fish, morphometrics, protein content, heavy metal pollution. ABSTRACT

212 marine fishery resources were recorded in the waters of Andhra Pradesh State. Morphometric data was provided for 20 edible fishery resources landing at the fishing harbour of Visakhapatnam. The harbour area is polluted due to influx of various industrial effluents and domestic sewage. In Pampus argenteus, P. chinensis and Parastromateus niger, the total protein content is $16.24-19.58 \%$. Further, arsenic concentration in muscle and gill portions individually or combined in all three of the species is highly negligible. Cadmium, mercury, and lead levels in the muscle and gills of these species are within or slightly above the recommended limits set by EU (2006) and FAO (2003), FAO/WHO (2011), MAFF, and FSSAI (2011) indicating that the consumption of these fishes is not harmful.

ZUSAMMENFASSUNG: Untersuchung der Meeresfischerei Ressourcen von Andhra Pradesh: ökologische Aspekte und morphometrische Angaben zu verbreiteten Meeresfischen des Visakhapatnam - Eiweißgehalt und Bioakkumulation von Schwermetallen in Pomfret Fischen.

In den Meeresbereichen des Bundesstaates Andhra Pradesh, wurden insgesamt 212 fischereiressourcen erfasst. Für 20 essbare Arten aus den fischereiressourcen, die im Fischereihafen von Visakhapatnam anlanden, wurden morphometrische Daten erfasst. Das Hafengebiet ist durch den Zufluss verschiedener Industrie- und häuslicher Abwässer dauernd verschmutzt. Bei Pampus argenteus, P. chinensis und Parastromateus niger beträgt der Gesamtproteingehalt 16,24-19,58\%. Auch ist die Arsenkonzentration in Muskel- und Kiemenanteilen einzeln oder in Kombination bei allen drei Arten sehr vernachlässigbar. Die Cadmium-, Quecksilber- und Bleiwerte in den Muskeln und Kiemen dieser Arten liegen innerhalb oder geringfügig über den von der EU (2006) und FAO (2003), FAO/WHO (2011), MAFF und FSSAI (2011) festgelegten Grenzwerten, was darauf hindeutet, dass der Verzehr dieser Fische nicht schädlich ist.

REZUMAT: Un studiu asupra resurselor piscicole marine din Andhra Pradesh: aspecte ecologice și morfometrice ale speciilor comune de pești marini din Visakhapatnam conținutul proteic și bioacumularea de metale grele la speciile familiei Bramidae.

212 resurse piscicole au fost înregistrate în apele marine ale statului Andhra Pradesh. $\mathrm{Au}$ fost furnizate date morfometrice pentru 20 de resurse piscicole comestibile din portul Visakhapatnam. Zona portuară este poluată de efluenți industriali și ape uzate menajere. În Pampus argenteus, $P$. chinensis și Parastromateus niger, conținutul total de proteine este între 16.24-19.58\%. Concentrația de arsenic în muşchi și branhii în toate speciile este neglijabilă. Nivelurile de cadmiu, mercur și plumb din mușchii și branhiile acestor specii se încadrează sau depășesc ușor limitele recomandate stabilite de UE (2006) și FAO (2003), FAO/OMS (2011), MAFF și FSSAI (2011), indicând faptul că consumul acestor pești nu este dăunător. 


\section{INTRODUCTION}

Phenotypic plasticity is important for fishes to respond adaptively to environmental changes by modification in their physiology and behaviour which in turn bring about changes in their morphology, reproduction or survival that mitigate the effects of environmental variation (Truman, 1999). The phenotype of an individual is largely unresponsive to fluctuating environmental conditions and is easily quantified while behavioural and physiological characters respond quickly to local conditions but fluctuate greatly during an individual's life-span (Rutherford et al., 1987; Crowl and Covich, 1990). Measurements of length-weight relationships are useful for the prediction of weight from length values, condition of fish, stock assessment, and estimation of biomass (Petrakis and Stergiou, 1995; Vaslet et al., 2007). Further, length-weight relationships also provide information about the stock composition, growth, life span, production, and mortality (Stergiou and Moutopoulos, 2001). Phenotypic features of fishes such as total length, standard length, head length, depth of the body and weight, are important for use as a valid method to identify the specimens of fish collected with their systematic morphology (Karunanidhi et al., 2017).

Fishes have been recognized as good accumulators of organic and inorganic pollutants (Gado and Midany, 2003; Curtean-Bănăduc et al., 2020). Age of fish, lipid content in the tissue, and mode of feeding are important factors that affect the accumulation of heavy metals in fishes. Fish diagnoses are often used to detect and monitor these heavy metal contaminations in aquatic ecosystems (Nadal et al. 2004; Kar et al., 2008). Heavy metals concentration of aquatic habitats has increased due to intense anthropogenic activities in recent years. Because of this condition, aquatic organisms are exposed to elevated levels of metals, which threaten the health of aquatic organisms as well as humans. Heavy metal accumulation levels in fishes from various aquatic environments have been widely reported (Zubcov et al., 2008; Khoshnood and Khoshnood, 2013; Taiwo et al., 2019). Fishes with heavy metal accumulation levels exceeding the stipulated limits if consumed by humans, then these metals may induce many health problems (Yilmaz, 2003; Papagiannis et al., 2004; Dalman et al., 2006). Fishes through respiration, adsorption, and ingestion can accumulate metals. The feeding habits and the rate of resorption are also important factors in metal accumulation. Benthic fish species have higher metal levels than pelagic species as the metal accumulation of sediments and detritus are many times higher than living organisms and water. Since the biological and ecological demands of fishes are different, the metal concentration levels of species are also different accordingly. Metal accumulation ratios of species depend on the water environment in which they are caught, season, and their trophic levels, sexes, and sizes. (Atlindag and Yigit, 2005; Yilmaz and Yilmaz, 2007) Metal concentration levels among various tissues and organs of the same species are also different. Metal accumulation capacity of muscle of fishes is lower than other tissues and organs such as skin, gill, intestine, and liver (Dural et al., 2007). Heavy metal levels in a fish are related to its living environment, feeding behaviour, and foraging habitats (Yi and Zhang, 2012; Rajeshkumar et al., 2018;). Some studies have suggested that the foraging habitat is a strong predictor for variations in heavy metal concentrations in fish (Goutte et al., 2015). Freshwater fishes tend to accumulate heavy metals in their organs more than marine fishes. The reason for such a difference between the two types of habitats is that freshwater fishes tend to lose salts and gain water. On the other hand, marine fishes tend to gain salts and lose water (Nikinmaa, 2014). Heavy metals are important due to their toxicity and ability to bioaccumulate in aquatic habitats and organisms (Miller et al., 2002; Astratinei and Varduca, 2008; Iepure and Selescu, 2009; Aziz et al., 2011; Akköz, 2016). Heavy metals tend to accumulate in the aquatic environment because they cannot be degraded, this leads to environmental problems and human exposure (Donati, 2018). 
Fish is a very important food source for humans, providing essential fatty acids like omega-3, proteins, vitamins, and minerals. Despite its nutritive value, its consumption brings an abundance of potential hazards for humans (Gado and Midany, 2003). Fish is at the top of the aquatic food chains and can accumulate large amounts of toxic elements like heavy metals (i.e. lead, mercury, cadmium, chromium, and arsenic) which can induce death when they concentrate too much in organisms' body. They have the tendency to accumulate in various organs and muscle tissue of fish. Contaminated fish enters the human body through consumption and causes health hazards (Nwabunike, 2016). Some heavy metals (i.e. mercury, cadmium, and lead) are toxic to fishes even at low concentrations, whereas others (i.e. zinc, copper, and cobalt) are biologically essential but become toxic at high concentrations (Amundsen et al., 1997). Some heavy metals such as arsenic, cadmium, lead, and mercury are of major environmental concern as they can cause severe health implications for humans and other living organisms. Moreover, some elements such as chromium, nickel, copper, and zinc that play essential roles in life activities become toxic in excess amounts (Huang et al., 2019).

Fish faunal diversity relates to genotypes with fish populations to species within a fish community to species across water regimes (Burton et al. 1992) and constitute a half of the total number of vertebrates in the entire world. About 2,500 species out of approximately a total of 22,000 fish species have been reported to be found in India. Of these, 1,570 species occur in marine waters (Jayaram 1999; Kar 2007, 2013, 2019). However, edible fish species inventory in each Indian State is not accurate. Since the inventory for the marine fish species of the coastline of Andhra Pradesh State is not available, there is an urgent need for this. Further, Visakhapatnam City has a coastline that extends about $132 \mathrm{~km}$ and has significant economic activity yielding out of the fishermen population associated with fishing activity. The area is polluted due to fast growing urbanization, industrialization, and tourism activities. Fishes caught from the coastal waters are sold in local fish markets regularly. But, there is no record of fish species that are common and commercially important and this situation warrants documenting the common and commercially important fish species sold in the local markets.

With this backdrop, the inventory of edible marine fish species in the study area and some common and commercially important edible marine fish species landing at Visakhapatnam Fishing Harbour/local fish markets have been separately provided. The ecological characters (biotype complex and feeding habitat) and morphological characters (total length/mantle length, standard length, head length, body depth, and weight) for all edible marine fish species landing at Visakhapatnam Fishing Harbour/local fish markets have been noted. The price (low, medium, and high) and importance (commercial/non-commercial) for each fish species have also been noted. Gears used for capturing the fishes that are captured from Visakhapatnam coast have been provided. The edible marine fish species exported through the Visakhapatnam Sea Food Export Trade Center have been listed along with the fish load exported by each exporting company. Further, protein content in muscle portion and heavy metals (cadmium, lead, mercury, and arsenic) in muscle and gill portions of three fish species, Pampus argenteus (Euphrasen, 1788), P. chinensis (Euphrasen 1788) (Stromateidae) and Parastromateus niger (Bloch, 1795) (Carangidae) collected from the Visakhapatnam Fishing Harbor have been assessed. These fish species are consumed by the inhabitants living in and around the study area. The assessment of heavy metals is important to understand the risks of fish contaminated with these metals on human health. Therefore, this knowledge is imperative to create awareness among consumers of these fishes and to monitor heavy metal concentrations regularly to control marine pollution and reduce its impact on human health. 


\section{MATERIAL AND METHODS}

\section{Study area}

The State of Andhra Pradesh, India, lies between $12^{\circ} 41^{\prime}$ and $19.07^{\circ} \mathrm{N}$ latitude and $77^{\circ}$ and $84^{\circ} 40^{\prime} \mathrm{E}$ longitude. It has a vast coastline which is a source of marine fishery resources that sustain local fishing communities by providing livelihood and provide seafood for most of the population in this State. Visakhapatnam $\left(17.6868^{\circ} \mathrm{N}\right.$ latitude and $83.2185^{\circ} \mathrm{E}$ longitude) is an important coastal belt that runs to more than $130 \mathrm{~km}$ and serves as a center for vibrant economic activity due to availability of various marine fishery resources and the dependence of fishermen on fishing activity for their livelihood. This place hosts a natural harbor and port which serves as a seat for local fish markets where edible marine fishes, crustaceans, and molluscans caught from the coastal waters are sold regularly. Further, it hosts busy shipping activities and a number of large and small scale industries. Coastal waters of Visakhapatnam are consistently polluted with treated and untreated industrial effluents, domestic sewage, material leakages despite the best efforts put in place by port and some industrial units.

\section{Inventory of marine fishery resources of Andhra Pradesh}

Field visits to different locations of coastal areas of Andhra Pradesh were made during 2018-2020 to record the marine species collected from coastal waters and sold in local fish markets. For this, personal visits were made to local fish markets to record the marine fishes sold locally. Further, secondary information available from Fisheries Department of Andhra Pradesh was also collected. Based on field and secondary information, an inventory of marine fishery resources was prepared for the entire state of Andhra Pradesh. In this inventory, the marine species were categorized into fishes, crustaceans, and molluscans, and accordingly the species were listed.

Common edible marine species landing/sold at Fishing Harbour and local fish markets of Visakhapatnam

Field visits to the fishing harbour and local fish markets were made at different times of the year during 2018-2020 to record the common edible fishes and molluscans collected from coastal waters of Visakhapatnam. The marine species were recorded family-wise and then their status was mentioned according to IUCN Red list.

Habitat, migration type, feeding habit, local and export value, and price value of common edible marine species landing/sold at Fishing Harbour and local fish markets of Visakhapatnam

Based on field visits, local fisheries department and secondary information from literature, habitat, migration type, feeding habit were provided for the fish and molluscan species sold at fishing harbour and local fish markets. Further, personal interviews with fishermen were conducted to elicit information on local and export value and price value of all these species. The information thus collected was recorded species-wise. This information was used to evaluate the importance of the habitat, migration type and feeding resources in enabling these species on a sustainable basis. Further, the information on the fish species exported and the price value were used to understand the importance of such species for providing livelihood, employment, and foreign exchange. The companies engaged in the export of commercially important marine species through the Sea Food Export Trade Center were noted. The quantity of each marine species exported (Mean and Standard Deviation) by each company once every 15 days was also recorded to know the value of exported species. 


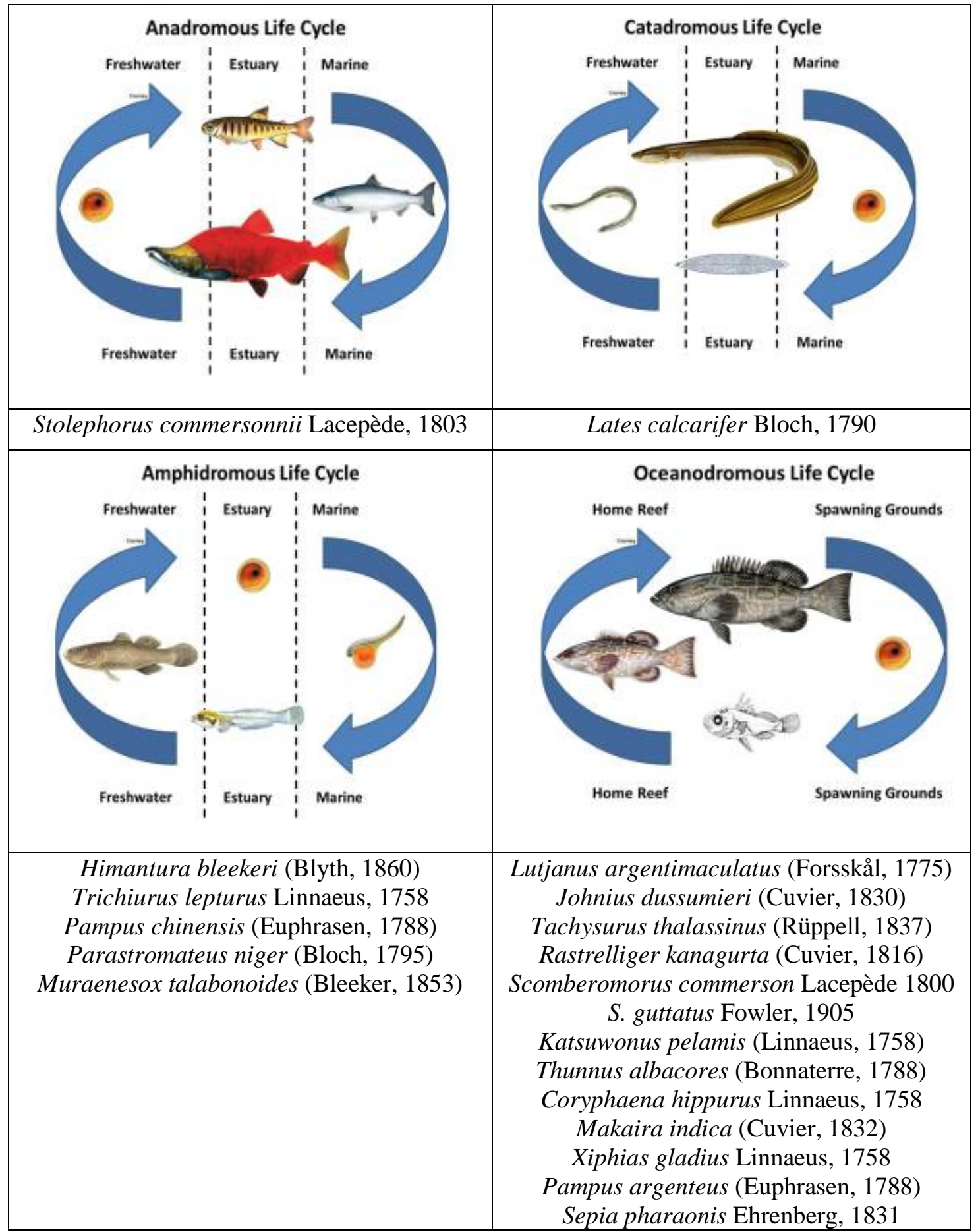

Figure 1: Migration types of marine

fish and molluscan species. 


\section{Gears used for capturing common edible marine species landing/sold at Fishing Harbor and local fish markets of Visakhapatnam}

Gears used for capturing marine species sold locally were recorded using the terminology followed in the International Standard Statistical Classification of Fishing Gear (ISSCFG, 2016). Information on gear types used was collected from fishermen who were involved in fish harvesting and this information was presented species-wise. Further, whether the gear-types change according to the season for capturing the same species was also collected. The period of occurrence, depth of occurrence (in meters), size at maturity and spawning season were also recorded for each captured species.

Description of morphometrics of common edible marine species landing/sold at Fishing Harbor and local fish markets of Visakhapatnam

Morphometric data was collected for all marine species sold locally. The standard length, total length, body depth, head length and weight were recorded for teleost species. Disc length, total length, and weight were recorded for the elasmobranch species. Mantle length, total length, and weight were recorded for the molluscan species. Length and depth measurements were recorded in centimeters while weight was recorded in grams. For all measurements, ten specimens were used for each species. The range, average, and standard deviation values were recorded for each species.

\section{Physico-chemical characteristics of coastal water of Visakhapatnam}

Ten samples of coastal surface water collected on different days in the month of May 2019 at 500 m away from Visakhapatnam harbor were analyzed for $\mathrm{pH}$, salinity, dissolved oxygen, and biological oxygen demand. $\mathrm{pH}$ was measured by a standardized $\mathrm{pH}$ meter. Dissolved oxygen (DO) and Biological Oxygen Demand (BOD) were determined using the Azide modification of the Winkler Method. Salinity was recorded using Salinity meter. Temperature was recorded with a precision thermometer. Based on the values recorded from all ten samples of surface sea water, range, mean, and standard deviation have been calculated.

\section{Morphological characteristics of Pomfret species}

Morphological characteristics of three Promfret fish species Pampus argenteus, Pampus chinensis and Parastromateus niger were described because they were selected for the assessment of protein content muscle portion and heavy metals arsenic, cadmium, mercury, and lead in muscle and gill parts. The morphological characters described enable to distinguish these three species from each other and also to identify easily in sea water and at selling point.

Determination of total protein content in the muscle tissue of Pampus argenteus, Pampus chinensis and Parastromateus niger

Ten fresh samples from ten different individuals of each fish species were collected from fishing harbor at Visakhapatnam. They were kept in cold iced box, taken to the laboratory and kept in a freezer before it was used for the determination of total protein content. The total protein content was determined by estimating the total nitrogen using Indian Standard Method (IS 7219-1973). In this method, the sample consisting of organic nitrogen was oxidized with concentrated sulphuric acid to convert it into ammonium sulphate in a micro Kjeldahl flask. Mercury was added to digestion mixture as a catalyst. The digest was diluted, made alkaline with anhydrous sodium sulphate and then distilled. Ammonia was liberated by adding an excess of alkali and was quantitatively distilled into a measured volume of standard sulphuric acid and total nitrogen was determined titrimetrically. The percent of protein content in the sample was calculated. This work was done at Vimta Labs Ltd., Visakhapatnam. 
Assessment of heavy metals in the muscle tissue and gill portions of Pampus argenteus, Pampus chinensis and Parastromateus niger

In digestion vessel, $0.5 \mathrm{~g}$ of homogenized muscle/gill samples were taken. Then, eight $\mathrm{ml}$ of concentrated nitric acid, one $\mathrm{ml}$ of concentrated hydrochloric acid and one $\mathrm{ml}$ of hydrogen peroxide were added to the homogenized samples. The digestion vessel was closed and then transferred to microwave digestion system at $190^{\circ} \mathrm{C}$ for 45 minutes. After completion of the digestion, digestion vessels were removed from the system, and cooled to room temperature, the sample was made up to $25 \mathrm{ml}$ with distilled water. The concentrations of heavy metals, arsenic, cadmium, mercury, and lead were determined using Inductively Coupled Plasma-Mass Spectrometry (ICP-MS) instrument.

Comparison of heavy metal concentrations analyzed in muscle and gill portions of Pomfret fish species with the permissible standards set out by different organizations

The detected heavy metal concentrations of arsenic, cadmium, mercury, and lead in muscle and gill portions of all the three Pomfret fish species were compared with the recommended limits permitted by European Union Regulations (EU) 2006, Food and Agriculture Organization (FAO)/World Health Organization (WHO) 2011, Ministry of Agriculture, Fisheries and Food (MAFF) and Food Safety and Standards Authority of India (FSSAI) as available.

\section{RESULTS}

Marine fin and shell fish species of coastline of Andhra Pradesh

An inventory of marine fish species of coastline of Andhra Pradesh included fin and shell fish species. Together, a total of 212 species have been integrated into this inventory (Tab. 1). Fin fishes include bony fishes while shellfishes include crustaceans and molluscs. Fin fishes included Elasmobranchs which are characteristically cartilaginous and Teleosts which are characteristically bony. Elasmobranchs were represented by 26 species consisting of sharks, skates, and rays. Sharks included 12 species belonging to five families. They were Chiloscyllium indicum (Hemiscyllidae), Rhincodon typus (Rhincodontidae), Stegostoma fasciatum (Stegostomatidae), Carcharhinus melanopterus, C. dussumieri, C. sorrah, Galeocerdo cuvieri, Rhizoprionodon acutus, Scoliodon laticaudus (Carcharhinidae), Eusphyra blochii, Sphyrna mokarran, and S. zygaena (Sphyrnidae). Skates included five species belonging to three families. They were Rhina ancylostoma, Rhynchobatus djiddensis (Rhinidae), Rhinobatus granulatus (Rhinobatidae), Anoxypristis cuspidate, and Pristis microdon (Pristidae). Rays were represented by nine species belonging to four families. They were Dasyatis zugei, Himantura bleekeri, H. uarnak (Dasyatidae), Aetomylaeus maculatus (Myliobatidae), Manta birostsis, Mobula diabolus (Mobulidae), Benthobatis moresbyi, Narcine brunnea, and Narcine timlei (Narcinidae). 
Table 1: Inventory of marine fin and shell fish species of coastline of Andhra Pradesh.

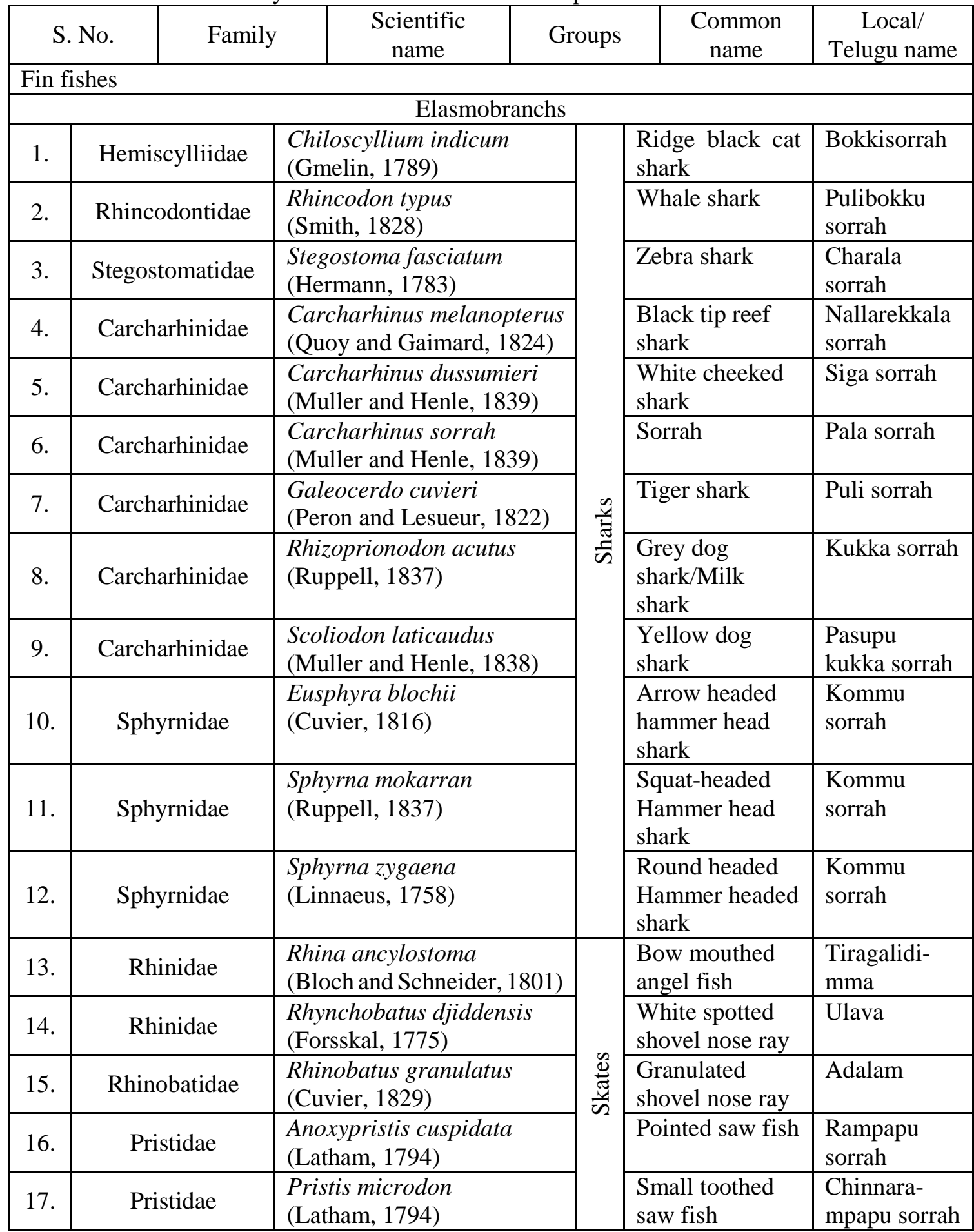


Table 1 (continued): Inventory of marine fin and shell fish species of coastline of Andhra Pradesh.

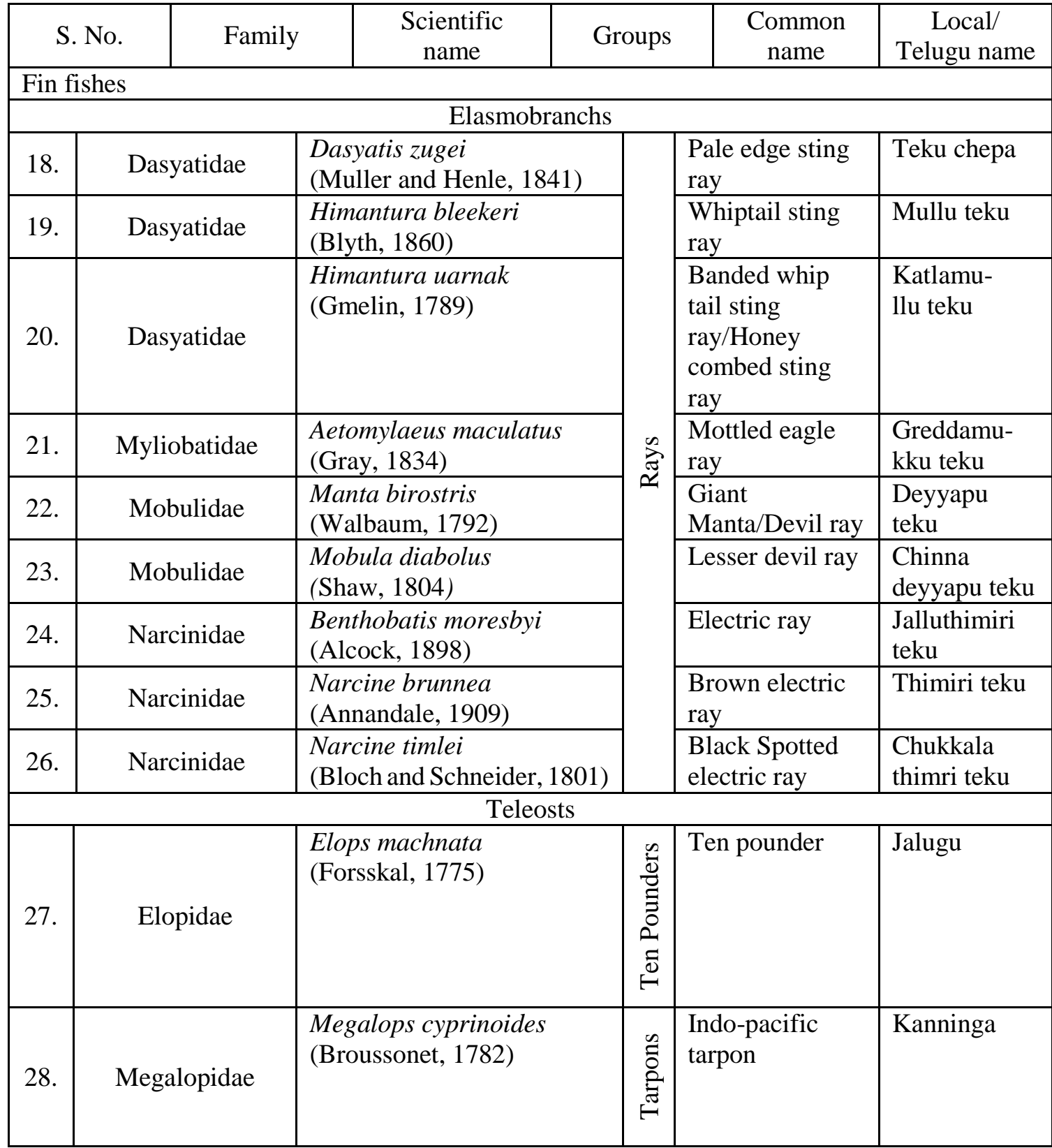


Table 1 (continued): Inventory of marine fin and shell fish species of coastline of Andhra Pradesh.

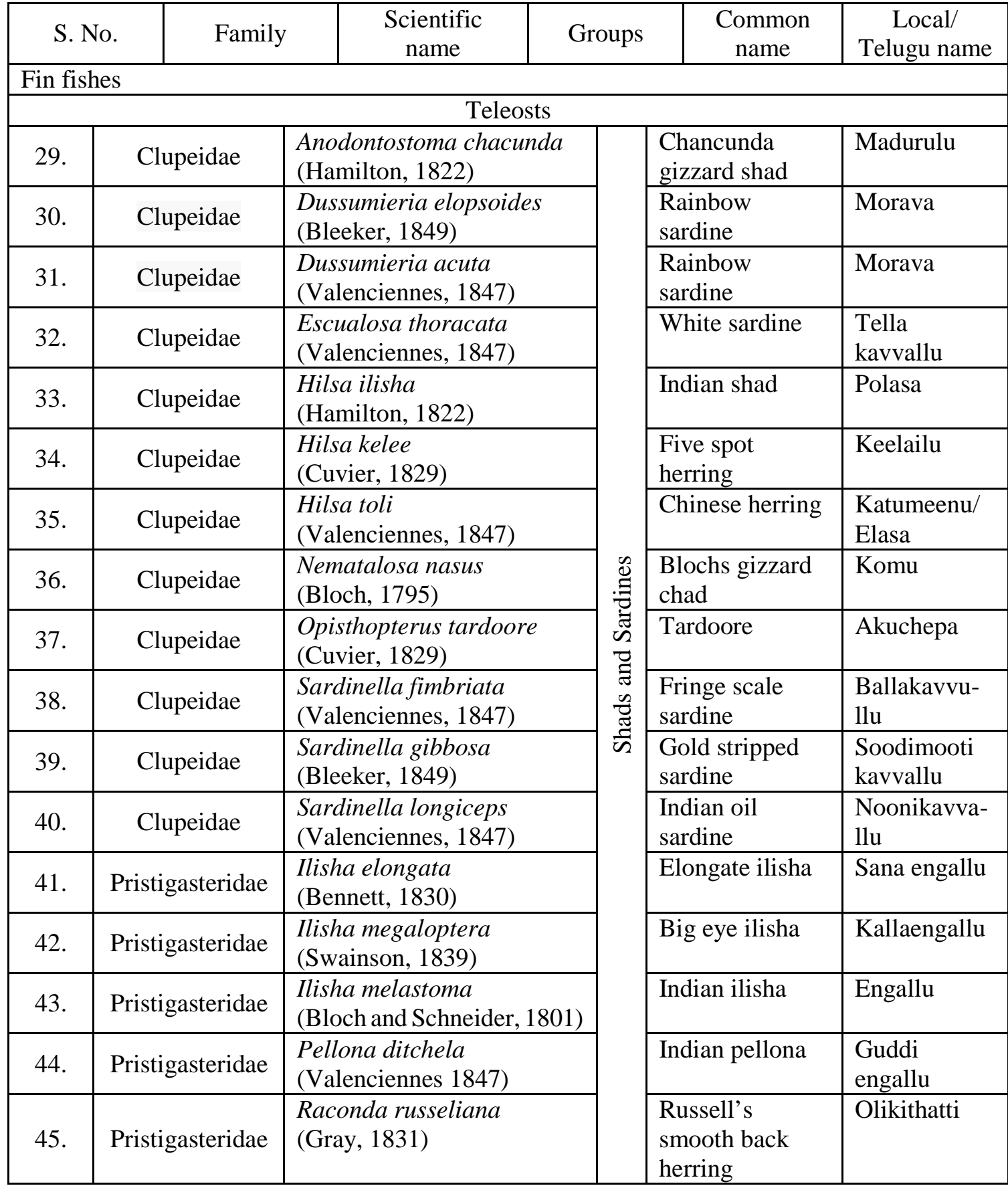


Table 1 (continued): Inventory of marine fin and shell fish species of coastline of Andhra Pradesh.

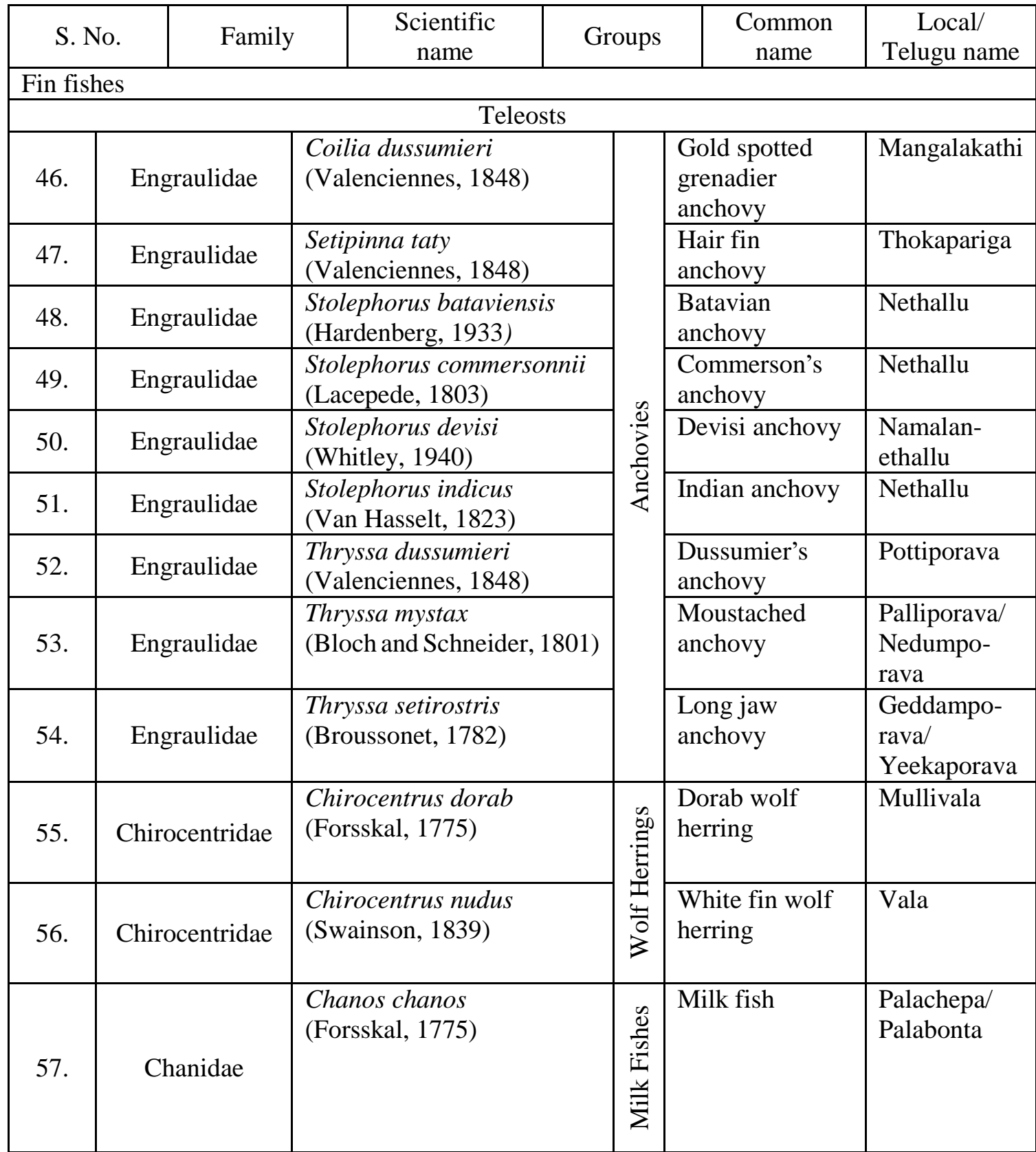


Table 1 (continued): Inventory of marine fin and shell fish species of coastline of Andhra Pradesh.

\begin{tabular}{|c|c|c|c|c|c|}
\hline \multirow[t]{2}{*}{ S. No. } & Family & $\begin{array}{l}\text { Scientific } \\
\text { name }\end{array}$ & Groups & $\begin{array}{c}\text { Common } \\
\text { name }\end{array}$ & $\begin{array}{c}\text { Local/ } \\
\text { Telugu name }\end{array}$ \\
\hline & \multicolumn{5}{|c|}{ Fin fishes } \\
\hline \multicolumn{6}{|c|}{ Teleosts } \\
\hline 58. & Synodontidae & $\begin{array}{l}\text { Saurida gracilis } \\
\text { (Quoy and Gaimard, 1824) }\end{array}$ & \multirow{4}{*}{ 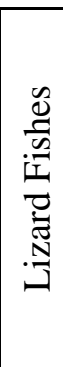 } & $\begin{array}{l}\text { Slender lizard } \\
\text { fish }\end{array}$ & $\begin{array}{l}\text { Sannabade- } \\
\text { metta }\end{array}$ \\
\hline 59. & Synodontidae & $\begin{array}{l}\text { Saurida tumbil } \\
\text { (Bloch, 1795) }\end{array}$ & & \begin{tabular}{|l} 
tish \\
Greater lizard \\
\end{tabular} & $\begin{array}{l}\text { Pedda } \\
\text { bademetta }\end{array}$ \\
\hline 60. & Synodontidae & $\begin{array}{l}\text { Saurida undosquamis } \\
\text { (Richardson, 1848) }\end{array}$ & & \begin{tabular}{|l} 
fish \\
Brush tooth \\
lizard fish
\end{tabular} & Bademetta \\
\hline 61. & Synodontidae & $\begin{array}{l}\text { Trachinocephalus myops } \\
\text { (Forster, 1801) }\end{array}$ & & $\begin{array}{l}\text { Blunt nose } \\
\text { lizard fish }\end{array}$ & $\begin{array}{l}\text { Esakadondu- } \\
\text { lu }\end{array}$ \\
\hline 62. & Synodontidae & $\begin{array}{l}\text { Harpadon nehereus } \\
\text { (Hamilton, 1822) }\end{array}$ & 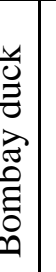 & Bombay duck & $\begin{array}{l}\text { Vanamatta/ } \\
\text { Kukkamatta }\end{array}$ \\
\hline 63. & Ariidae & $\begin{array}{l}\text { Tachysurus dussumieri } \\
\text { (Valenciennes, 1840) }\end{array}$ & \multirow{4}{*}{ 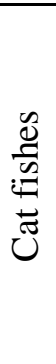 } & $\begin{array}{l}\text { Dussumier’s cat } \\
\text { fish }\end{array}$ & Penkijella \\
\hline 64. & Ariidae & $\begin{array}{l}\text { Tachysurus tenuispinis } \\
\text { (Day, 1877) }\end{array}$ & & $\begin{array}{l}\text { Thin spined cat } \\
\text { fish } \\
\end{array}$ & Nallajella \\
\hline 65. & Ariidae & $\begin{array}{l}\text { Tachysurus thalassinus } \\
\text { (Ruppell, 1837) }\end{array}$ & & Giant cat fish & Tella jella \\
\hline 66. & Bagridae & $\begin{array}{l}\text { Macrones gulio } \\
\text { (Hamilton, 1822) }\end{array}$ & & $\begin{array}{l}\text { Long whiskers } \\
\text { cat fish }\end{array}$ & Jellakoyyallu \\
\hline 67. & Plotosidae & $\begin{array}{l}\text { Plotosus anguillaris } \\
\text { (Bloch, 1794) }\end{array}$ & \multirow{2}{*}{ 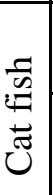 } & Stripped cat fish & Silthi \\
\hline 68. & Plotosidae & $\begin{array}{l}\text { Plotosus canius } \\
\text { (Hamilton, 1822) }\end{array}$ & & Canine cat fish & Engilai \\
\hline 69. & Anguillidae & $\begin{array}{l}\text { Anguilla bicolor bicolor } \\
\text { (McClelland, 1844) }\end{array}$ & & Level finned & Nalla pamu \\
\hline 70. & Anguillidae & $\begin{array}{l}\text { Anguilla nebulosa } n . \\
\text { (McClelland, 1844) }\end{array}$ & $\begin{array}{l}0 \\
0 \\
0 \\
0\end{array}$ & $\begin{array}{l}\text { Long finned } \\
\text { eel }\end{array}$ & Nalla pamu \\
\hline 71. & Muraenesocidae & $\begin{array}{l}\text { Muraenesox talabonoides } \\
\text { (Bleeker, 1853) }\end{array}$ & 它 & $\begin{array}{c}\text { Indian pike } \\
\text { conger }\end{array}$ & Tella pamu \\
\hline 72. & Muraenesocidae & $\begin{array}{l}\text { Muraenesox cinereus } \\
\text { (Forsskal, 1775) }\end{array}$ & $\underset{0}{\infty}$ & $\begin{array}{c}\text { Dagger } \\
\text { toothed pike } \\
\text { conger }\end{array}$ & Pasupu pamu \\
\hline
\end{tabular}


Table 1: Inventory of marine fin and shell fish species of coastline of Andhra Pradesh.

\begin{tabular}{|c|c|c|c|c|c|c|}
\hline S. No. & Famil & $\begin{array}{l}\text { Scientific } \\
\text { name }\end{array}$ & \multicolumn{2}{|c|}{ Groups } & $\begin{array}{l}\text { Common } \\
\text { name }\end{array}$ & $\begin{array}{c}\text { Local/ } \\
\text { Telugu name }\end{array}$ \\
\hline \multicolumn{7}{|c|}{ Fin fishes } \\
\hline \multicolumn{7}{|c|}{ Teleosts } \\
\hline 73. & Belonidae & \multicolumn{2}{|c|}{$\begin{array}{l}\text { Strongylura crocodilus } \\
\text { (Peron and Lesueur, 1821) }\end{array}$} & 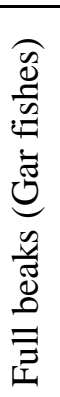 & $\begin{array}{l}\text { Fork tail } \\
\text { aligator gar }\end{array}$ & Kadurulu \\
\hline 74. & Hemiramphidae & \multicolumn{2}{|c|}{$\begin{array}{l}\text { Hemirhamphus marginatus } \\
\text { (Forsskal, 1775) }\end{array}$} & 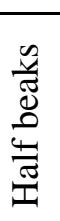 & Barred half beak & Kadurulu \\
\hline 75. & Exocoetidae & \multicolumn{2}{|c|}{$\begin{array}{l}\text { Cypselurus cyanopterus } \\
\text { (Valenciennes, 1847) }\end{array}$} & \multirow{2}{*}{ 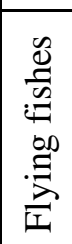 } & $\begin{array}{l}\text { Blue spot flying } \\
\text { fish }\end{array}$ & Gopirangulu \\
\hline 76. & Exocoetidae & \multicolumn{2}{|l|}{$\begin{array}{l}\text { Exocoetus volitans } \\
\text { (Linnaeus, 1758) }\end{array}$} & & $\begin{array}{l}\text { Two winged } \\
\text { flying fish }\end{array}$ & Thooreegalu \\
\hline 77. & Fistulariidae & \multicolumn{2}{|l|}{$\begin{array}{l}\text { Fistularia petimba } \\
\text { (Lacepede, 1803) }\end{array}$} & \multirow{2}{*}{ 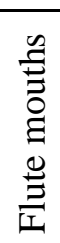 } & $\begin{array}{l}\text { Smooth flute } \\
\text { mouth }\end{array}$ & Kolasi \\
\hline 78. & Fistulariidae & \multicolumn{2}{|l|}{$\begin{array}{l}\text { Fistularia villosa } \\
\text { (Klunzinger, 1871) }\end{array}$} & & $\begin{array}{l}\text { Rough flute } \\
\text { mouth }\end{array}$ & Garukukolasi \\
\hline 79. & Sphyraenidae & \multicolumn{2}{|l|}{$\begin{array}{l}\text { Sphyraena jello } \\
\text { (Cuvier, 1829) }\end{array}$} & \multirow{2}{*}{ 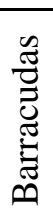 } & \begin{tabular}{|l} 
Banned \\
barracuda
\end{tabular} & $\begin{array}{l}\text { Charala } \\
\text { seelapotu }\end{array}$ \\
\hline 80. & Sphyraenidae & \multicolumn{2}{|l|}{$\begin{array}{l}\text { Sphyraena obtusata } \\
\text { (Cuvier, 1829) }\end{array}$} & & $\begin{array}{l}\text { Obtuse } \\
\text { barracuda }\end{array}$ & Seelapotu \\
\hline 81. & Mugilidae & \multicolumn{2}{|l|}{$\begin{array}{l}\text { Liza tade } \\
\text { (Forsskal, 1775) }\end{array}$} & \multirow{3}{*}{ 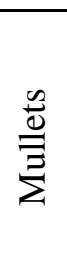 } & $\begin{array}{l}\text { Tade grey } \\
\text { mullet }\end{array}$ & Kaniselu \\
\hline 82. & Mugilidae & \multicolumn{2}{|l|}{$\begin{array}{l}\text { Mugil cephalus } \\
\text { (Linnaeus, 1758) }\end{array}$} & & $\begin{array}{l}\text { Flat head grey } \\
\text { mullet }\end{array}$ & $\begin{array}{l}\text { Kattachepa/ } \\
\text { Bontalu }\end{array}$ \\
\hline 83. & Mugilidae & \multicolumn{2}{|l|}{$\begin{array}{l}\text { Valamugil cunnesius } \\
\text { (Valenciennes, 1836) }\end{array}$} & & $\begin{array}{l}\text { Long fin grey } \\
\text { mullet }\end{array}$ & Kaniselu \\
\hline
\end{tabular}


Table 1 (continued): Inventory of marine fin and shell fish species of coastline of Andhra Pradesh.

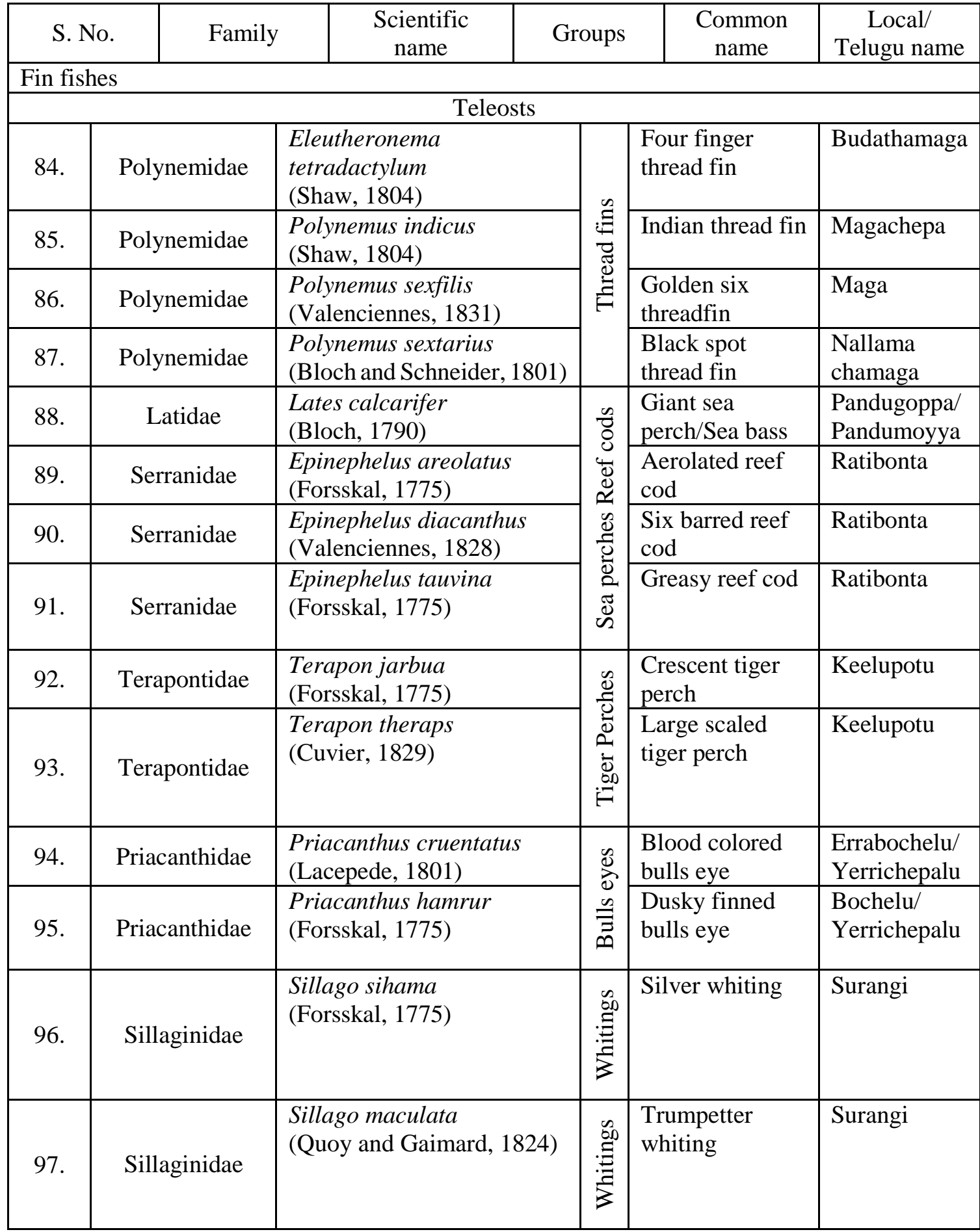


Table 1 (continued): Inventory of marine fin and shell fish species of coastline of Andhra Pradesh.

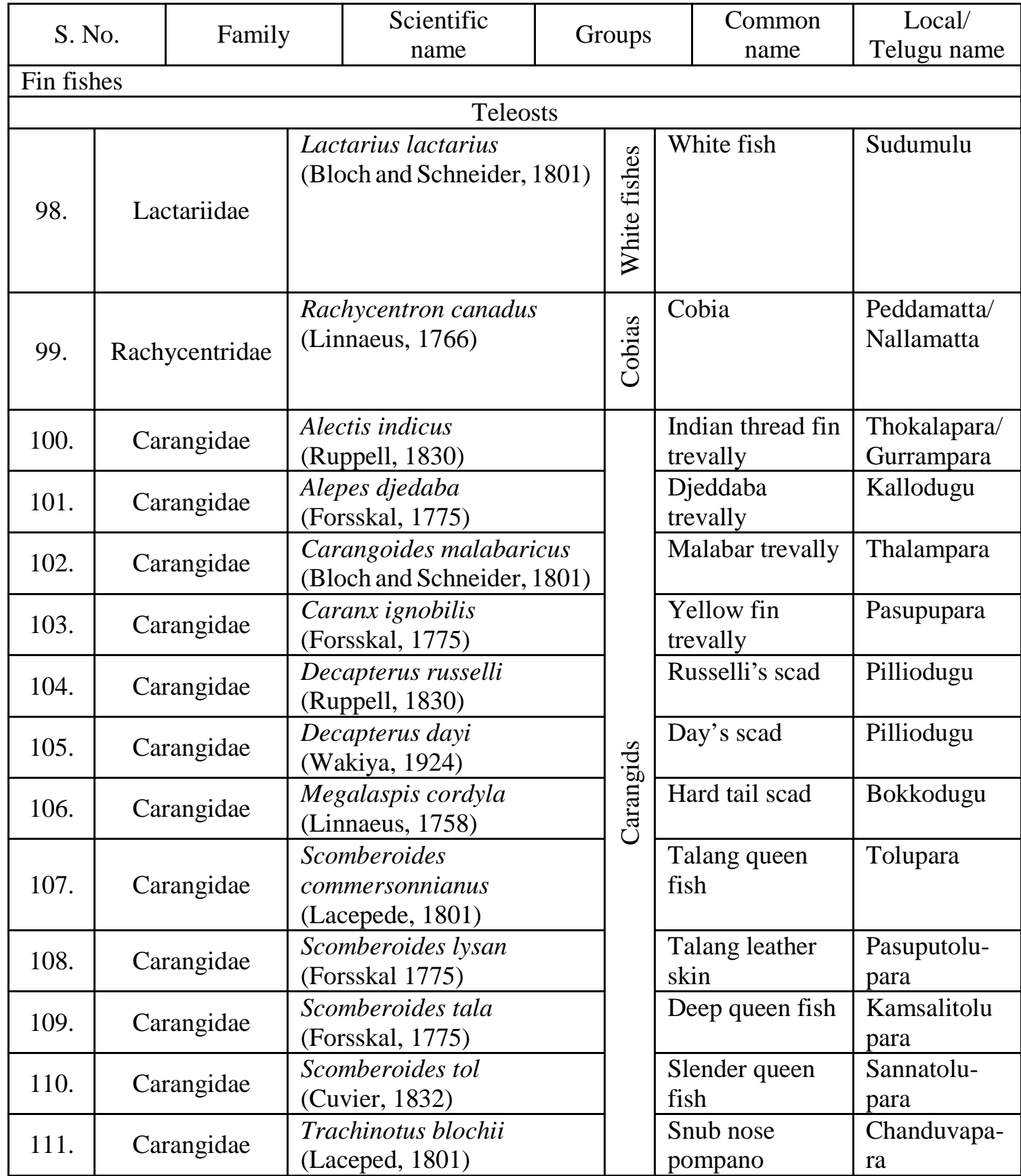


Table 1 (continued): Inventory of marine fin and shell fish species of coastline of Andhra Pradesh.

\begin{tabular}{|c|c|c|c|c|c|c|}
\hline S. 1 & Famil & $\begin{array}{l}\text { Scientific } \\
\text { name }\end{array}$ & \multicolumn{2}{|c|}{ Groups } & $\begin{array}{c}\text { Common } \\
\text { name }\end{array}$ & $\begin{array}{c}\text { Local/ } \\
\text { Telugu name }\end{array}$ \\
\hline \multicolumn{7}{|c|}{ Fin fishes } \\
\hline \multicolumn{7}{|c|}{ Teleosts } \\
\hline 112. & Menidae & \begin{tabular}{|l|} 
Mene maculata \\
(Bloch and Schneider,
\end{tabular} & & 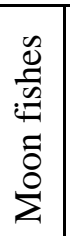 & Moon fish & $\begin{array}{l}\text { Chukkala } \\
\text { chanduva }\end{array}$ \\
\hline 113. & Coryphaenidae & $\begin{array}{l}\text { Coryphaena hippurus } \\
\text { (Linnaeus, 1758) }\end{array}$ & & 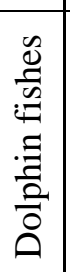 & $\begin{array}{l}\text { Common } \\
\text { dolphin } \\
\text { fish/Mahi Mahi }\end{array}$ & Avalosu \\
\hline 114. & Lutjanidae & $\begin{array}{l}\begin{array}{l}\text { Lutjanus argentimacu } \\
\text { (Forsskal, 1775) }\end{array} \\
\end{array}$ & & \multirow{2}{*}{ 营 } & \multirow{2}{*}{\begin{tabular}{|l|}
$\begin{array}{l}\text { Mangrove red } \\
\text { snapper }\end{array}$ \\
Johns snapper
\end{tabular}} & $\begin{array}{l}\text { Ratigoraka/ } \\
\text { Yerragoraka }\end{array}$ \\
\hline 115. & Lutjanidae & $\begin{array}{l}\text { Lutjanus johnii } \\
\text { (Bloch, 1792) }\end{array}$ & & & & $\begin{array}{l}\text { Samarlu/ } \\
\text { Yerragoraka }\end{array}$ \\
\hline 116. & Nemipteridae & $\begin{array}{l}\text { Nemipterus delagoae } \\
\text { (Smith, 1941) }\end{array}$ & \multirow{3}{*}{\multicolumn{2}{|c|}{ 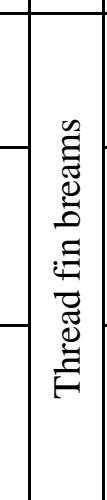 }} & $\begin{array}{l}\text { Delagoan } \\
\text { threadfin bream }\end{array}$ & $\begin{array}{l}\text { Yerra } \\
\text { gulivindalu/ } \\
\text { Gulivindalu }\end{array}$ \\
\hline 117. & Nemipteridae & $\begin{array}{l}\text { Nemipterus japonicus } \\
\text { (Bloch, 1791) }\end{array}$ & & & $\begin{array}{l}\text { Japanese thread } \\
\text { fin bream }\end{array}$ & $\begin{array}{l}\text { Yerra } \\
\text { gulivinda/ } \\
\text { Bandi } \\
\text { gulivindalu }\end{array}$ \\
\hline 118. & Nemipteridae & \begin{tabular}{|l}
$\begin{array}{l}\text { Nemipterus mesoprior } \\
\text { (Bleeker, 1853) }\end{array}$ \\
\end{tabular} & & & $\begin{array}{l}\text { Red filament } \\
\text { thread fin bream }\end{array}$ & $\begin{array}{l}\text { Yerra } \\
\text { gulivindalu/ } \\
\text { Bandiguli- } \\
\text { vindalu }\end{array}$ \\
\hline 119. & Lobotidae & $\begin{array}{l}\begin{array}{l}\text { Lobotes surinamensis } \\
\text { (Bloch, 1790) }\end{array} \\
\end{array}$ & & 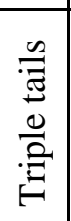 & Brown triple tail & Maata \\
\hline
\end{tabular}


Table 1: Inventory of marine fin and shell fish species of coastline of Andhra Pradesh.

\begin{tabular}{|c|c|c|c|c|c|}
\hline S. No. & Family & $\begin{array}{c}\text { Scientific } \\
\text { name }\end{array}$ & Groups & $\begin{array}{l}\text { Common } \\
\text { name }\end{array}$ & $\begin{array}{c}\text { Local/ } \\
\text { Telugu name }\end{array}$ \\
\hline \multicolumn{6}{|c|}{ Fin fishes } \\
\hline \multicolumn{6}{|c|}{ Teleosts } \\
\hline 120. & Leiognathidae & $\begin{array}{l}\text { Gazza minuta } \\
\text { (Bloch, 1795) }\end{array}$ & \multirow{7}{*}{ 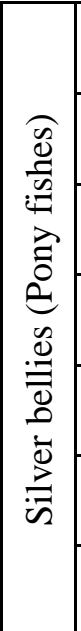 } & $\begin{array}{l}\text { Toothed pony } \\
\text { fish }\end{array}$ & Sudumu kara \\
\hline 121. & Leiognathidae & $\begin{array}{l}\text { Leiognathus bindus } \\
\text { (Valenciennes, 1835) }\end{array}$ & & $\begin{array}{l}\text { Orange fin } \\
\text { pony fish }\end{array}$ & Bendu kara \\
\hline 122. & Leiognathidae & $\begin{array}{l}\text { Leiognathus dussumieri } \\
\text { (Valenciennes, 1835) }\end{array}$ & & $\begin{array}{l}\text { Dussumier's } \\
\text { pony fish }\end{array}$ & Charala kara \\
\hline 123. & Leiognathidae & $\begin{array}{l}\text { Leiognathus equulus } \\
\text { (Forsskal, 1775) }\end{array}$ & & $\begin{array}{l}\text { Common pony } \\
\text { fish }\end{array}$ & Chanduva kara \\
\hline 124. & Leiognathidae & $\begin{array}{l}\text { Leiognathus splendens } \\
\text { (Cuvier 1829) }\end{array}$ & & $\begin{array}{l}\text { Splendid pony } \\
\text { fish } \\
\end{array}$ & Tatta kara \\
\hline 125. & Leiognathidae & Secutor insidiator & & $\begin{array}{l}\text { Pugnose pony } \\
\text { fish }\end{array}$ & Chukka kara \\
\hline 126. & Leiognathidae & Secutor ruconius & & $\begin{array}{l}\text { Deep pug nose } \\
\text { pony fish }\end{array}$ & $\begin{array}{l}\text { Chinni chukka } \\
\text { kara }\end{array}$ \\
\hline 127. & Gerreidae & Gerres filamentosus & \multirow{2}{*}{ 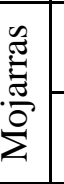 } & $\begin{array}{l}\text { Whip fin } \\
\text { mojarra }\end{array}$ & $\begin{array}{l}\text { Jaggari/Vada- } \\
\text { gava }\end{array}$ \\
\hline 128. & Gerreidae & $\begin{array}{l}\text { Pentaprion longimanus } \\
\text { (Cantor, 1849) }\end{array}$ & & $\begin{array}{l}\text { Long fin } \\
\text { mojarra }\end{array}$ & $\begin{array}{l}\text { Karnigavala/ } \\
\text { Varipindikudelu }\end{array}$ \\
\hline 129. & Haemulidae & $\begin{array}{l}\text { Pomadasys hasta } \\
\text { (Bloch, 1790) }\end{array}$ & 离 & $\begin{array}{l}\text { Lined silver } \\
\text { grunt }\end{array}$ & Pandugoraka \\
\hline 130. & Haemulidae & $\begin{array}{l}\text { Pomadasys maculatus } \\
\text { (Bloch 1793) }\end{array}$ & 胥 & Blotched grunt & Karipi \\
\hline 131. & Sciaenidae & $\begin{array}{l}\text { Atrobucca nibe } \\
\text { (Jordan and Thompson, 1911) }\end{array}$ & & $\begin{array}{l}\text { Black mouthed } \\
\text { croaker }\end{array}$ & $\begin{array}{l}\text { Karrimooti } \\
\text { gorasa }\end{array}$ \\
\hline 132. & Sciaenidae & $\begin{array}{l}\text { Johnieops vogleri } \\
\text { (Bleeker, 1853) }\end{array}$ & & Drab croaker & Gorasa \\
\hline 133. & Sciaenidae & $\begin{array}{l}\text { Johnius carutta } \\
\text { (Bloch, 1793) }\end{array}$ & & Karut croaker & $\begin{array}{l}\text { Nalla gorasa/ } \\
\text { Charagorassa }\end{array}$ \\
\hline 134. & Sciaenidae & $\begin{array}{l}\text { Johnius dussumieri } \\
\text { (Cuvier, 1830) }\end{array}$ & & $\begin{array}{l}\text { Bearded } \\
\text { croaker }\end{array}$ & $\begin{array}{l}\text { Geddam } \\
\text { gorassa }\end{array}$ \\
\hline 135. & Sciaenidae & $\begin{array}{l}\text { Kathala axillaris } \\
\text { (Cuvier, 1830) }\end{array}$ & 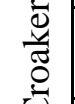 & $\begin{array}{l}\text { Kathala } \\
\text { croaker }\end{array}$ & Palli gorassa \\
\hline 136. & Sciaenidae & $\begin{array}{l}\text { Nibea maculata } \\
\text { (Bloch and Schneider, 1801) }\end{array}$ & 5 & $\begin{array}{l}\text { Blotches } \\
\text { croaker }\end{array}$ & $\begin{array}{l}\text { Nallamachala } \\
\text { gorassa }\end{array}$ \\
\hline 137. & Sciaenidae & $\begin{array}{l}\text { Otolithes ruber } \\
\text { (Bloch and Schneider, 1801) }\end{array}$ & & $\begin{array}{l}\text { Tiger toothed } \\
\text { croaker }\end{array}$ & $\begin{array}{l}\text { Villigorassa/ } \\
\text { Palla gorassa }\end{array}$ \\
\hline 138. & Sciaenidae & $\begin{array}{l}\text { Pennahia macrophthalmus } \\
\text { (Bleeker, 1849) }\end{array}$ & & $\begin{array}{l}\text { Big eye } \\
\text { croaker }\end{array}$ & Kalla gorassa \\
\hline 139. & Sciaenidae & $\begin{array}{l}\text { Protonibea diacanthus } \\
\text { (Lacepede, 1802) }\end{array}$ & & $\begin{array}{l}\text { Spotted } \\
\text { croaker }\end{array}$ & Pandu gorassa \\
\hline
\end{tabular}


Table 1 (continued): Inventory of marine fin and shell fish species of coastline of Andhra Pradesh.

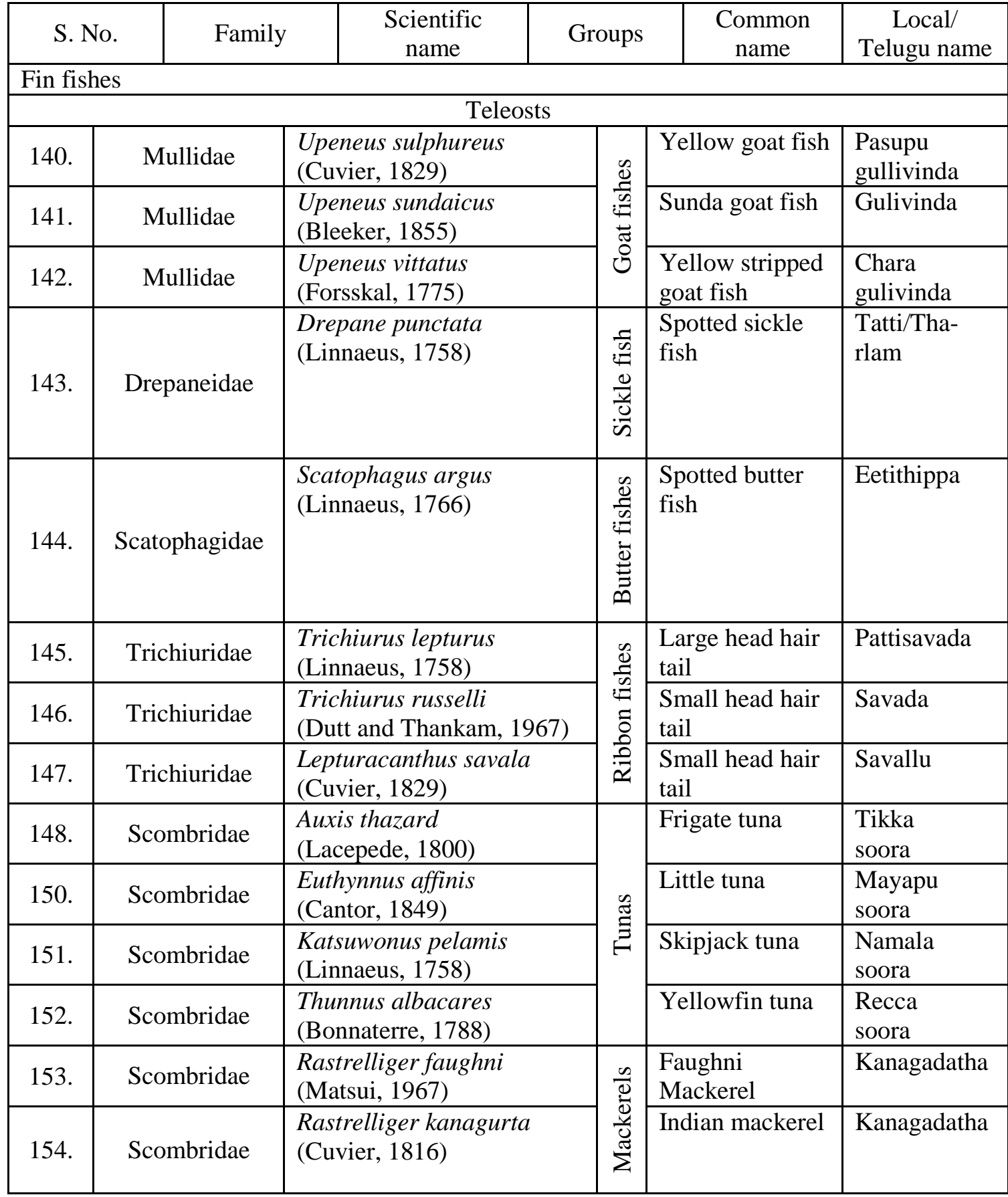


Table 1 (continued): Inventory of marine fin and shell fish species of coastline of Andhra Pradesh.

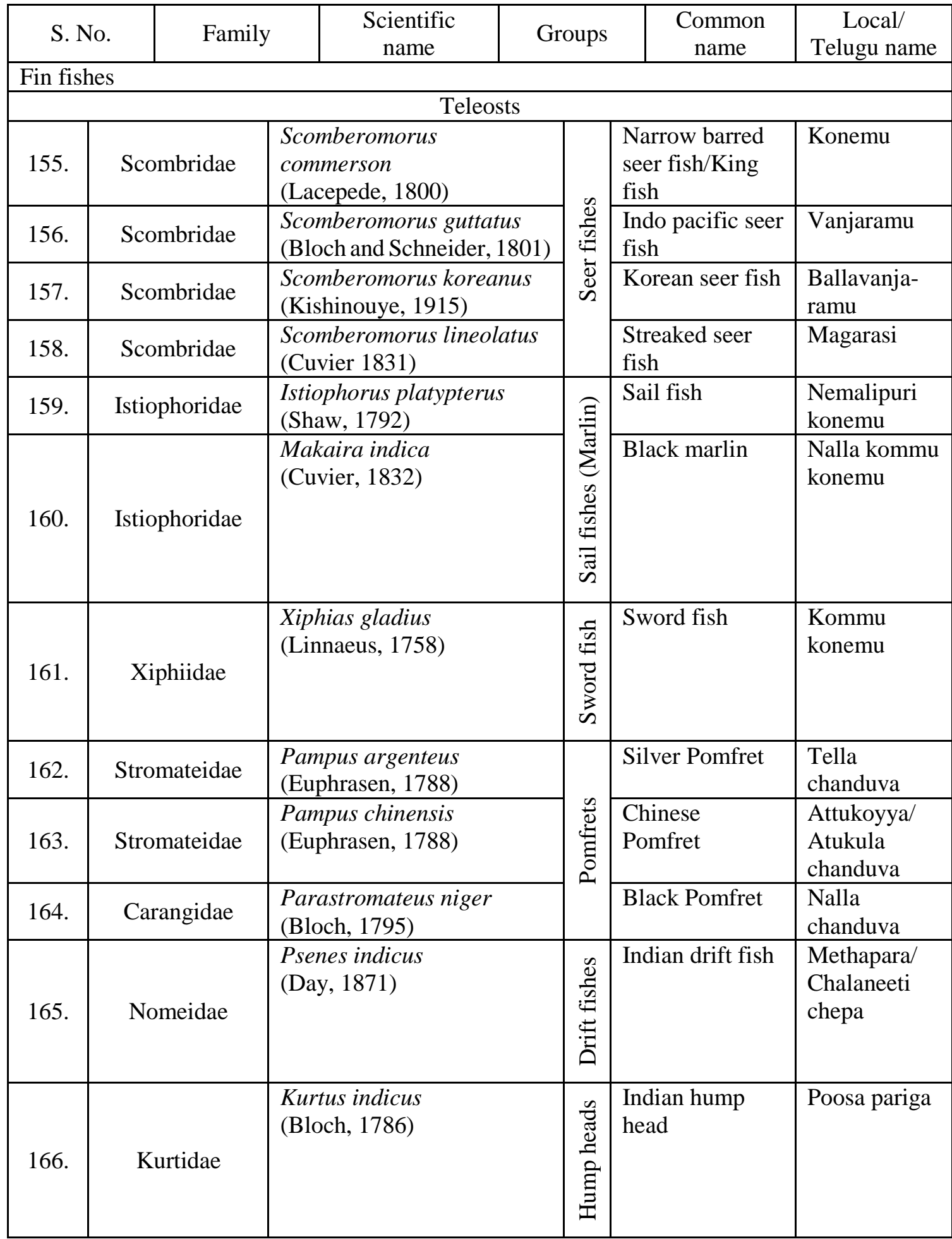


Table 1 (continued): Inventory of marine fin and shell fish species of coastline of Andhra Pradesh.

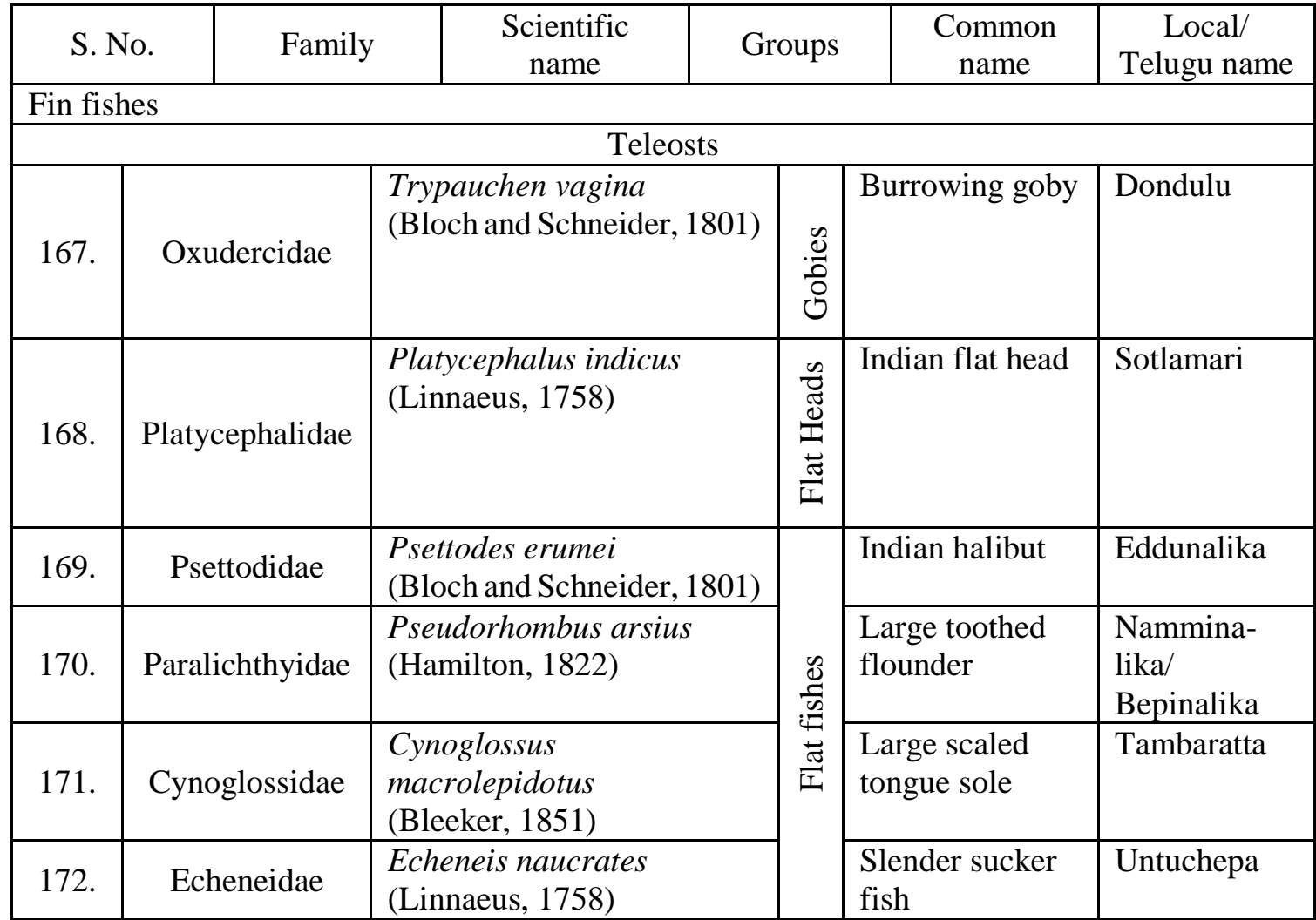


Table 1 (continued): Inventory of marine fin and shell fish species of coastline of Andhra Pradesh.

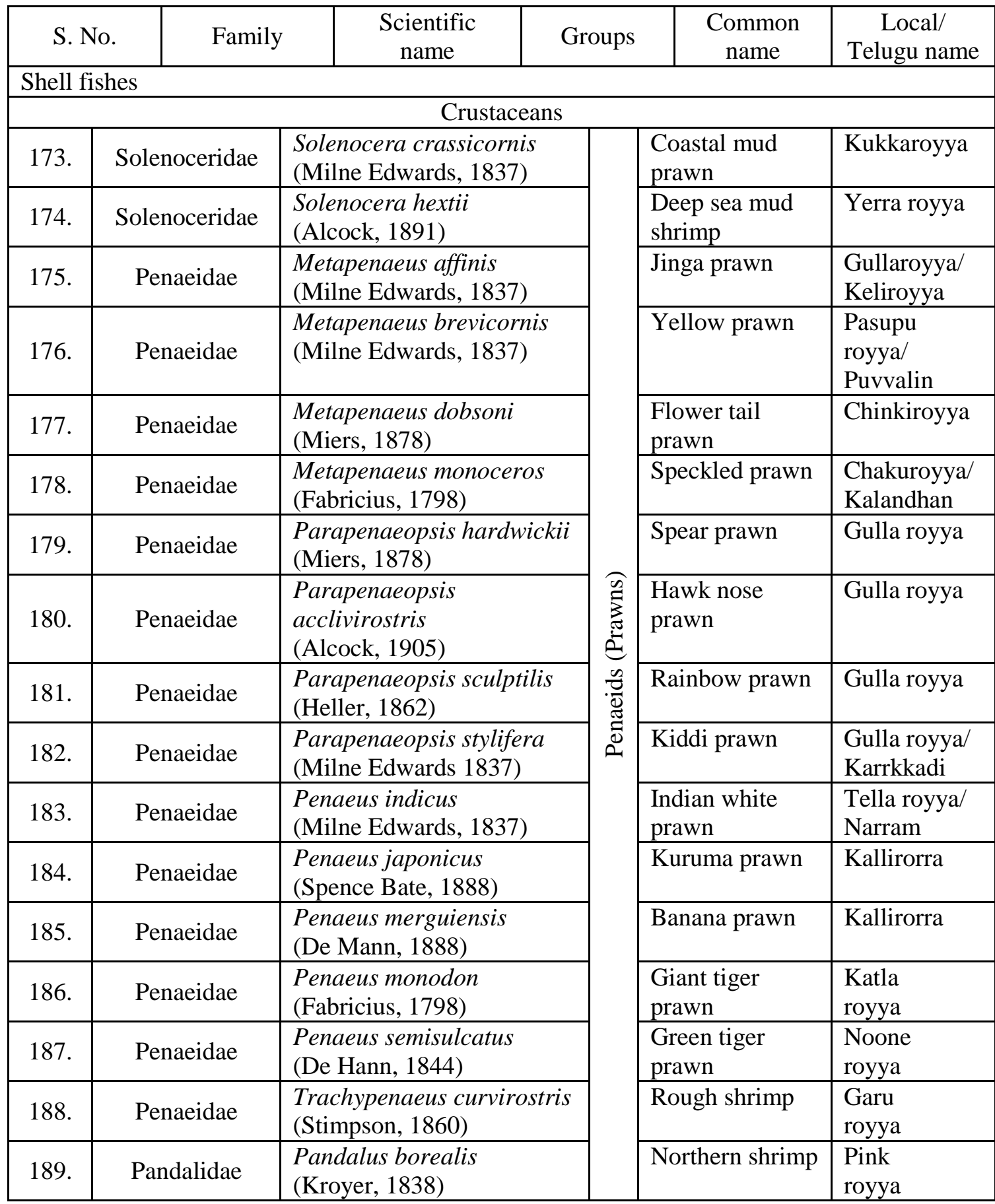


Table 1 (continued): Inventory of marine fin and shell fish species of coastline of Andhra Pradesh.

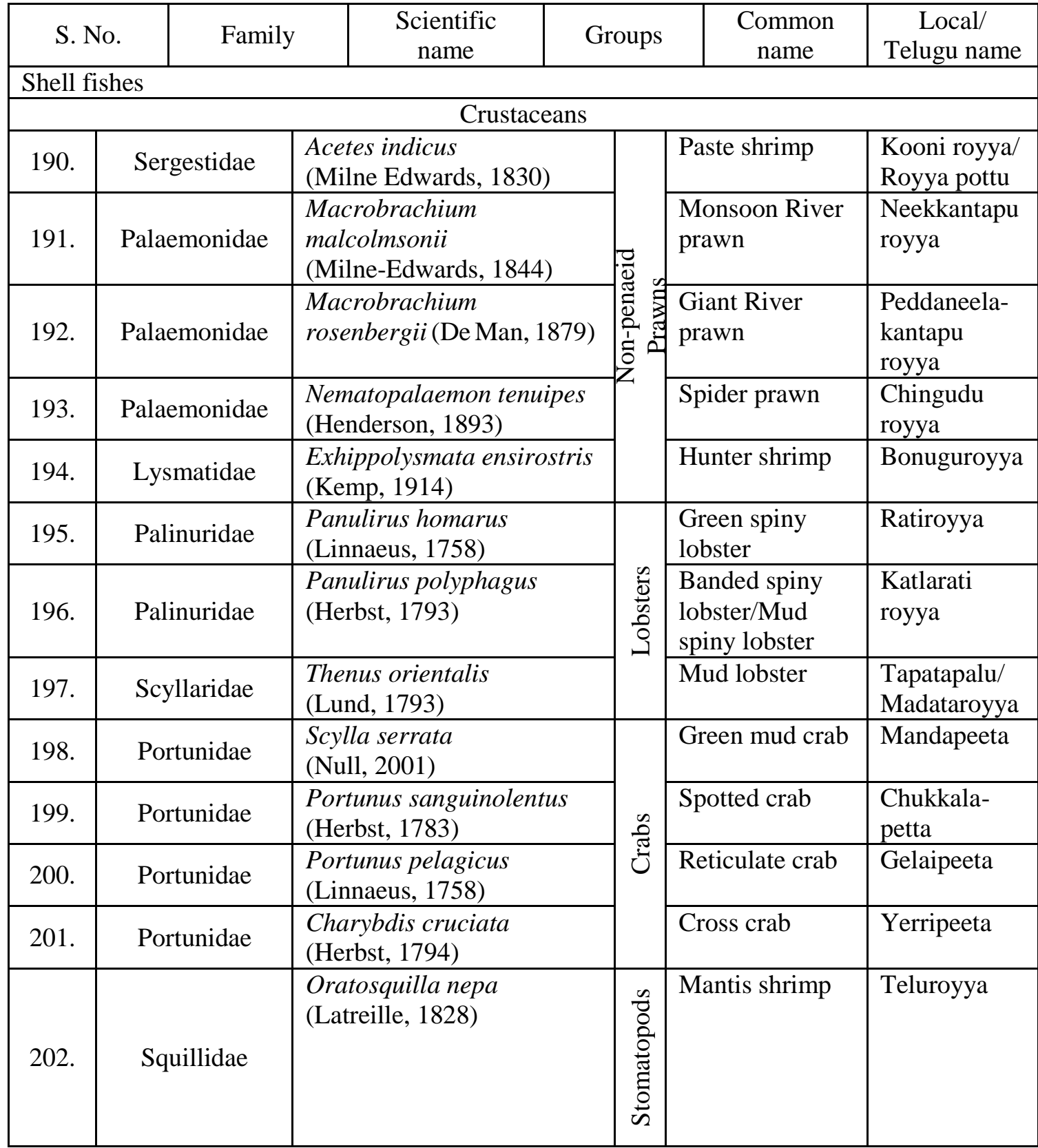


Table 1: Inventory of marine fin and shell fish species of coastline of Andhra Pradesh.

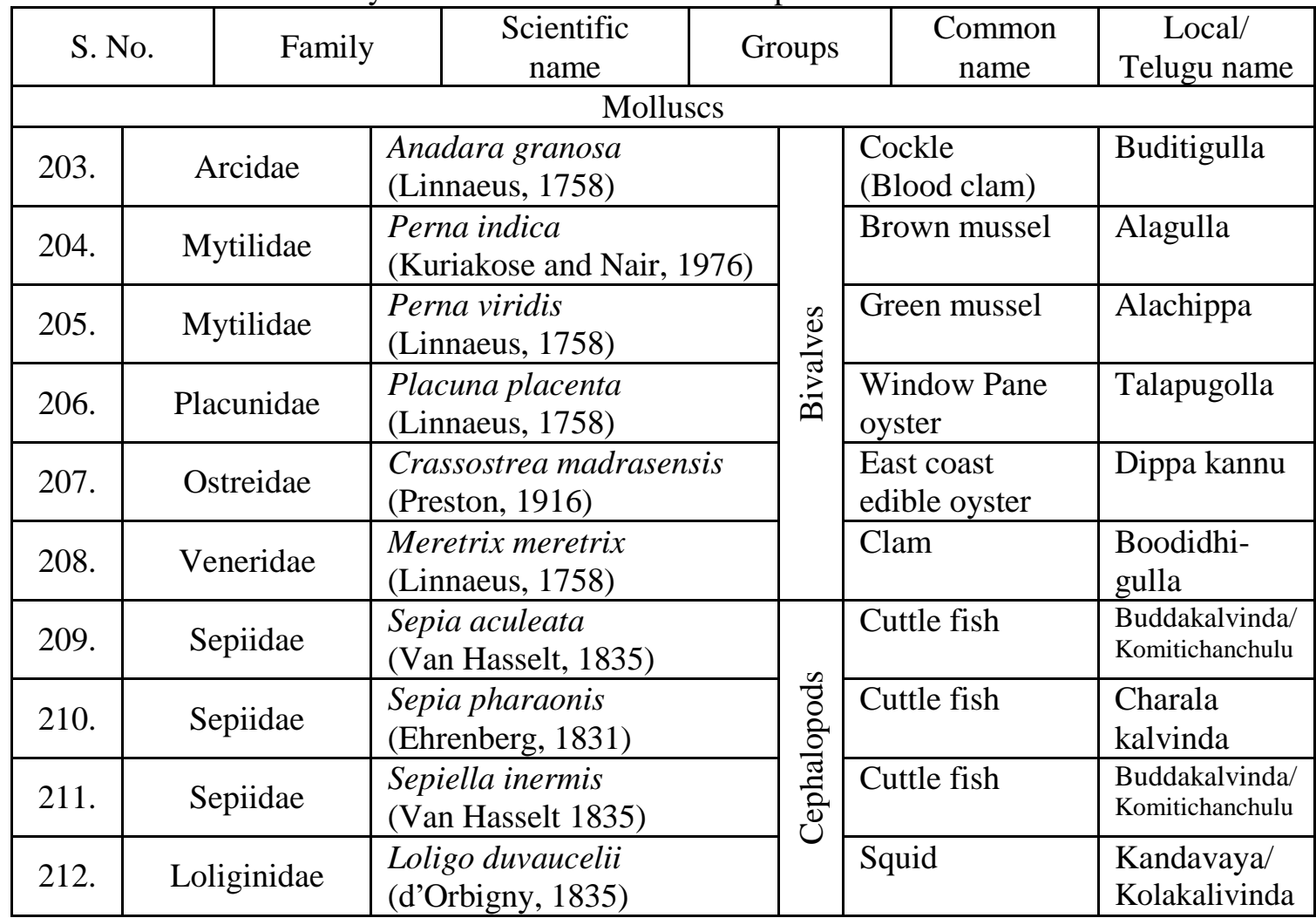

Teleosts were represented by 146 species consisting of Ten Pounders, Tarpons, Shads, Sardines, Anchovies, Wolf Herrings, Milk Fishes, Lizard Fishes, Bombay Ducks, Cat fishes, Cat fish eels, Eels and Congers, Full beaks (Gar fishes), Half beaks, Flying fishes, Flute mouths, Barracudas, Mullets, Thread fins, Sea perches, Reef cods, Tiger Perches, Bulls eye, Whitings, White fishes, Cobias, Carangids, Moon fishes, Dolphin fishes, Snappers, Thread fin breams, Triple tails, Silver bellies (Pony fishes), Mojarras, Grunters, Croakers, Goat fishes, Sickle fishes, Butter fishes, Ribbon fishes, Tunas, Mackerels, Seer fishes, Sail fishes, Sword fishes, Pomfrets, Drift fishes, Hump heads, Gobies, Flat heads, and Flat fishes.

In Teleosts, Ten Pounders, and Tarpons included one species each, the former was Elops machnata (Elopidae) and the latter was Megalops cyprinoides (Megalopidae). Shads and sardines included 17 species belonging to two families. They were Anodontostoma chacunda, Dussumieria elopsoides, D. acuta, Escualosa thoracata, Hilsa ilisha, H. kelee, H. toli, Nematalosa nasus, Opisthopterus tardoore, Sardinella fimbriata, S. gibbosa, S. longiceps (Clupeidae), Ilisha elongata, I. megaloptera, I. melastoma, Pellona ditchela, and Raconda russeliana (Pristigasteridae). Anchovies included nine species and all belonging to only one family, Engraulidae. They were Coilia dussumieri, Setipinna taty, Stolephorus bataviensis, $S$. commersonnii, S. devisi, S. indicus, Thyrssa dussumieri, T. mystax, and T. setirostris. Wolf herrings included two species, Chirocentrus dorab and C. nudus, both belonging to Chirocentridae. Milk fish was represented by a single species, Chanos chanos which belonged to Chanidae. Lizard fishes included four species, Saurida gracilis, S. tumbil, S. undosquamis, and Trachinocephalus myops, all belonging to Synodontidae. Bombay Duck was represented 
by a single species, Harpadon nehereus which belonged to Synodontidae. Cat fishes included four species belonging to two families. They were Tachysurus dussumieri, T. tenuispinis, $T$. thalassinus (Ariidae), and Macrones gulio (Bagridae). Cat fish eels included two species, Plotosus anguillaris and P. canius, both belonging to Plotosidae. Eels and congers were represented by four species which belonged to two families. They were Anguilla bicolor bicolor, A. nebulosa nebulosa (Anguillidae), Muraenesox talabonoides, and M. cinereus (Myraenesocidae). Gar fishes and Half beaks were represented by one species each, the former was represented by Strongylura crocodilus (Belonidae) while the latter was represented by $H$. marginatus (Hemiramphidae). Flying fishes, Flute mouths and Barracudas were represented by two species each; the first type was represented by Cypselurus cyanopterus and Exocoetus volitans (Exocoetidae), the second type by Fistularia petimba and F. villosa (Fistularidae) and the last type by Sphyraena jello and S. obtusata (Sphyraenidae). Mullets included three species, Liza tade, Mugil cephalus, and Valamugil cunnesius, all three species belonged to Mugilidae family. Thread fins included four species, Eleutheronema tetradactylum, Polynemus indicus, $P$. sexfilis, and $P$. sextarius, all belonged to Polynemidae family. Sea perches were represented by a single species, Lates calcarifer which belonged to Latidae. Reef cods included three species, Epinephelus areolatus, E. diacanthus and E. tauvina, all three belonged to Serranidae. Tiger Perches were represented by two species, Terapon jarbua and T. theraps which belonged to Terapontidae. Bull eyes represented two species, Priacanthus cruentatus and $P$. hamrur, both belonged to Priacanthidae family. Whitings included two species, Sillago sihama and S. maculata which belonged to Sillaginidae family. White fishes and Cobias were represented by one species each, the former by Lactarius lactarius and the latter by Rachycentron canadus. Carangids included 12 species, Alectis indicus, Aepes djedaba, Carangoides malabaricus, Caranx ignobilis, Decapterus russelli, D. dayi, Megalaspis cordyla, Scomberoides commersonnianus, S. lysan, S. tala, S. tol, and Trachinotus blochii, all belonged to Carangidae family. Moon fishes and Dolphin fishes included one species each, the former was Mene maculata (Menidae family) and the latter was Coryphaena hippurus (Coryphaenidae family). Snappers included two species, Lutjanus argentimaculatus and $L$. johnii belonging to Lutjanidae. Thread fin breams included three species, Nemipterus delagoae, $N$. japonicas, and $N$. mesoprion, all three belonged to Nemipteridae family. Triple tails was represented by Lobotes surinamensis which belonged to Lobotidae family. Silver bellies included seven species, Gazza minuta, Leiognathus bindus, L. dussumieri, L. equulus, L. splendens, Secutor insidiator, and S. ruconius, all belonged to Leiognathidae family. Mojarras and Grunters, each were represented by two species; the former by Gerres filamentosus and Pentaprion longimanus (Gerreidae) while the latter by Pomadasys hasta and P. maculatus (Haemulidae). Croakers included nine species, Atrobucca nibe, Johnieops vogleri, J. carutta, J. dussumieri, Kathala axillaris, Nibea maculata, Otolithes ruber, Pennahia macrophthalmus, and Protonibea diacanthus, all belonged to Sciaenidae family only. Goat fishes included three species, Upeneus sulphureus, $P$. sundaicus, and $U$. vittatus, all belonged to Mullidae family. Sickle fishes and Butter fishes included one species each, the former was Drepano punctata (Drepanenidae) and the latter was Scatophagus argus (Scatophagidae). Ribbon fishes included three species, Trichiurus lepturus, T. russelli and Lepturacanthus savala, all belonged to Trichiuridae family. Tunas, Mackerels and Seer fishes were represented by Scombridae family. Tunas included five species, Auxis thazard, Euthynnus affinis, Katsuwonus pelamis, Thunnus albacores, and T. tonggol; Mackerels included two species, Rastrelliger faughni and R. kanagurta and Seer fishes included four species, Scomberomorus commerson, S. guttatus, S. koreanus, and S. lineolatus. Sail fishes included two species, Istiophorus platypterus and Makaira indica (Istiophoridae). Sword fish was represented by a 
single species, Xiphias gladius (Xiphiidae). Pomfrets included three species, Pampus argenteus, P. chinensis (Stromateidae), and Parastromateus niger (Carangidae). Drift fishes, Hump heads, Gobies, and Flat heads, each was represented by one species, Psenes indicus (Nomeidae), Kurtus indicus (Nomeidae), Trypauchen vagina (Oxudercidae) and Platycephalus indicus (Platycephalidae). Flat fishes included four species, Psettodes erumei, P. arsius (Paralichthyidae), Cynoglossus macrolepidotus (Cynoglossidae), and Echeneis naucrates (Echeneidae).

In Shell fishes, crustaceans included 30 species which represented 10 families while molluscs included 10 species which represented seven families. The crustaceans represented Penaeids, Non-penaeids, Lobsters, Crabs, and Stomatopods while molluscs represented Bivalves and Cephalopods. Penaeids included 17 species which represented three families. They were Solenocera crassicornis, S. hextii (Solenoceridae), Metapenaeus affinis, M. brevicornis, M. dobsoni, M. monoceros, Parapenaeopsis hardwickii, P. acclivirostris, $P$. sculptilis, $P$. stylifera, Penaeus indicus, $P$. japonicus, $P$. merguiensis, $P$. monodon, $P$. semisulcatus, Trachypenaeus curvirostris (Penaeidae), and Pandalus borealis (Pandalidae). Non-penaeids included five species which represented three families. They were Acetes indicus (Sergestidae), Macrobrachium malcolmsonii, M. rosenbergii, Nematopalaemon tenuipes (Palaemonidae), and Exhippolysmata ensirostris (Lysmatidae). Lobsters included three species, Panulirus homarus, P. polyphagus (Palinuridae), and Thenus orientalis (Scyllaridae). Crabs included four species, Scylla serrata, Portunus sanguinolentus, $P$. pelagicus, Charybdis cruciata (Portunidae). Stomatopods included one species, Oratosquilla nepa (Squillidae).

In Molluscs, Bivalves included six species belonging to five families: Anadara granosa (Arcidae), Perna indica, P. viridis (Mytilidae), Placuna placenta (Placunidae), Crassostrea madrasensis (Ostreidae), Meretrix meretrix (Veneridae).

Cephalopods included four species, Sepia aculeata, S. pharaonis, Sepiella inermis (Sepiidae), and Loligo duvauceliii (Loliginidae).

The fin and shell-fish species listed in table 1 were found to be used according to their availability; some species are seasonal while some others are aseasonal. Field observations and personal queries with locals across the coastline of Andhra Pradesh indicated that some species are common, some others are uncommon and still some others are rare. Further, some species are of local value while some others are of export value indicating that marine fishery resources are important as seafood, employment provider, and foreign exchange earner.

Common marine fin- and shell-fish species landing/sold at Fishing Harbour and local fish markets of Visakhapatnam: At Fishing Harbour and local markets of Visakhapatnam, twenty marine species were commonly captured and sold depending on their availability. The cartilaginous fishes (Elasmobranchs) included one species, Himantura bleekeri (Dasyatidae). Bony fishes (Teleosts) included 18 species belonging to 13 families. They were Lutjanus argentimaculatus (Latjanidae), Johnius dussumieri (Sciaenidae), Tachysurus thalassinus (Ariidae), Stolephorus commersonii (Engraulidae), Trichiurus lepturus (Trichiuridae), Lates calcarifer (Latidae), Rastrelliger kanagurta, Scomberomorus commerson, S. guttatus, Katsuwonus pelamis, Thunnus albacares (Scombridae), Coryphaena hippurus (Coryphaenidae), Makaira indica (Istiophoridae), Xiphias gladius (Xiphiidae), Pampus argenteus, P. chinensis (Stromateidae), Parastromateus niger (Carangidae), and Muraenesox talabonoides (Muraenesocidae). One mollusc species, Sepia pharaonis (Cephalopod: Sepiidae family) was commonly sold (Tab. 2). All twenty species are shown in figures 2-7. 


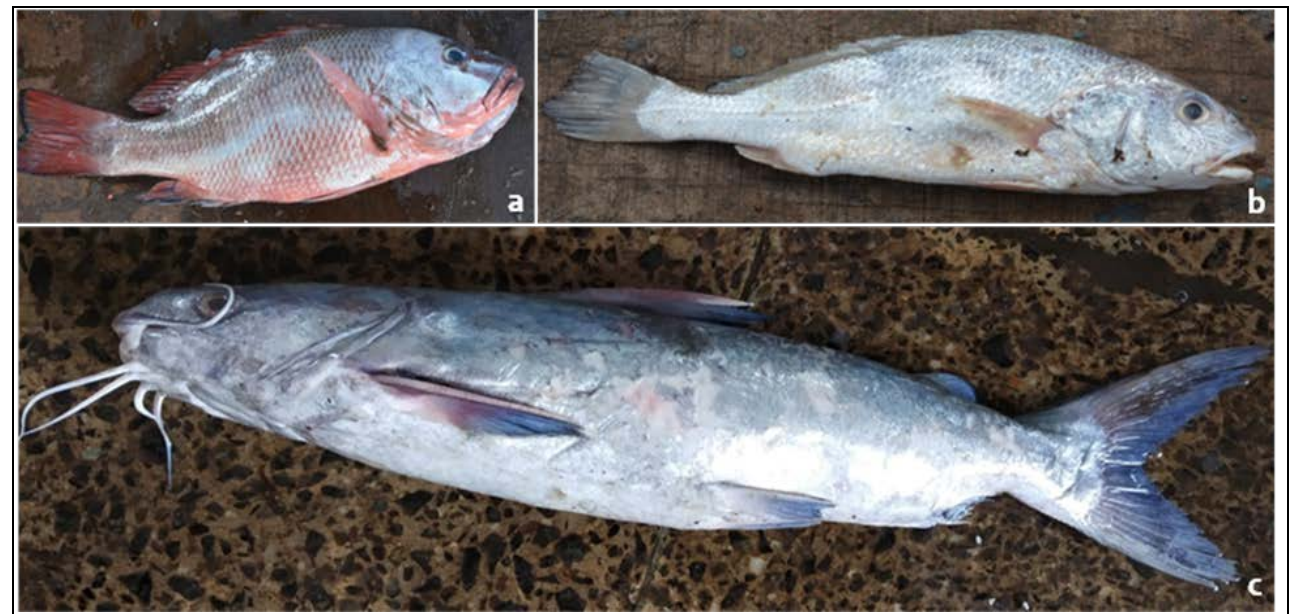

Figure 2a: Lutjanus argentimaculatus, b. Johnius dussumieri, c. Tachysurus thalassinus.

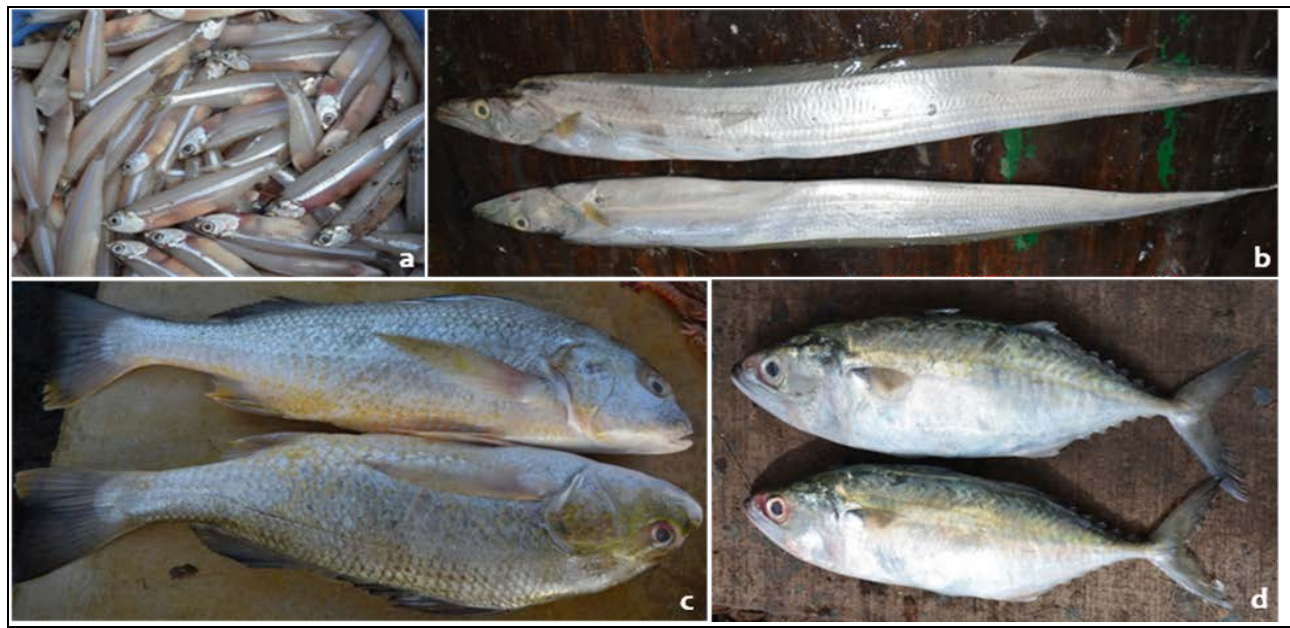

Figure 3a: Stolephorus commersonnii, b. Trichiurus lepturus, c. Lates calcarifer, d. Rastrelliger kanagurta.

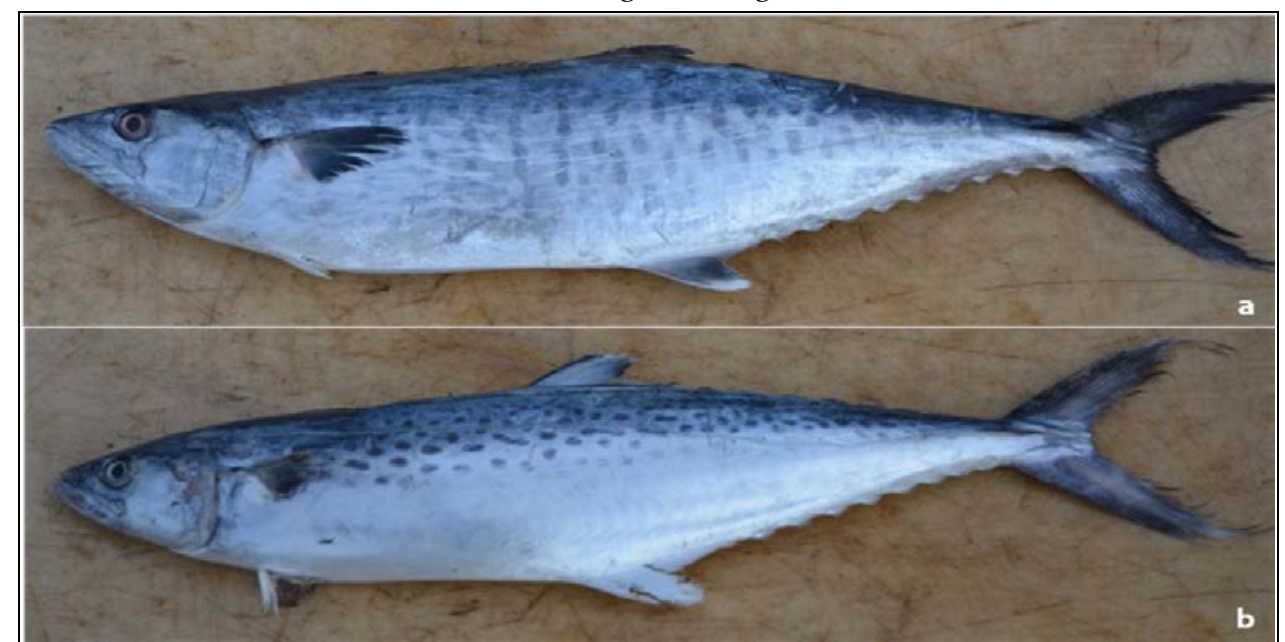

Figure 4a: Scomberomorus commerson, b. Scomberomorus guttatus. 


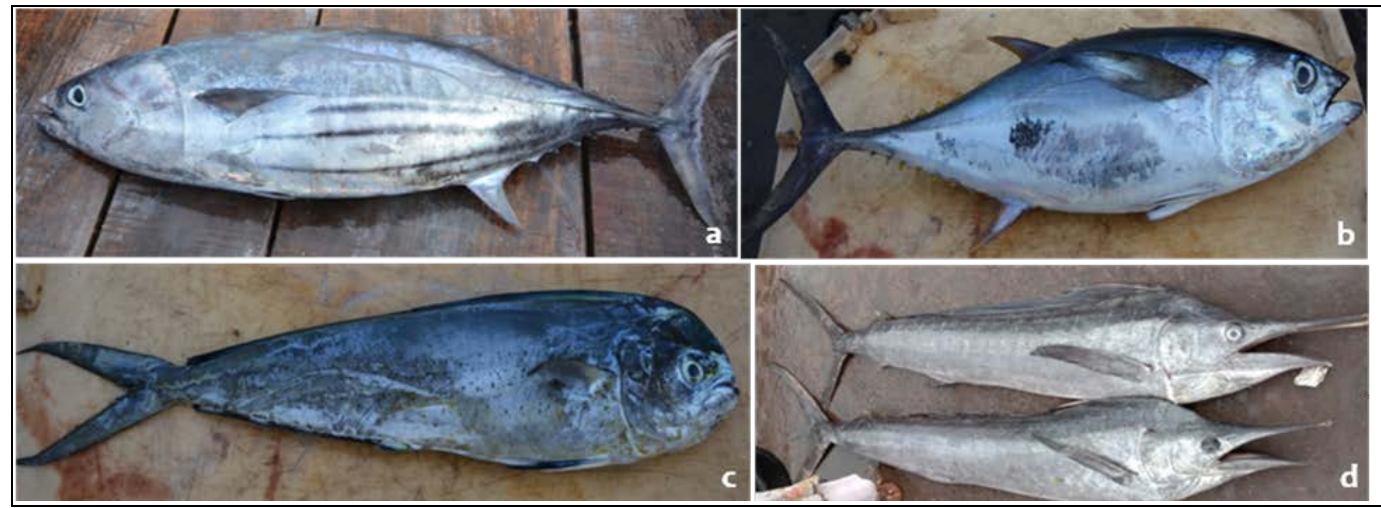

Figure 5a: Katsuwonus pelamis, b. Thunnus albacares, c. Coryphaena hippurus, d. Makaira indica.

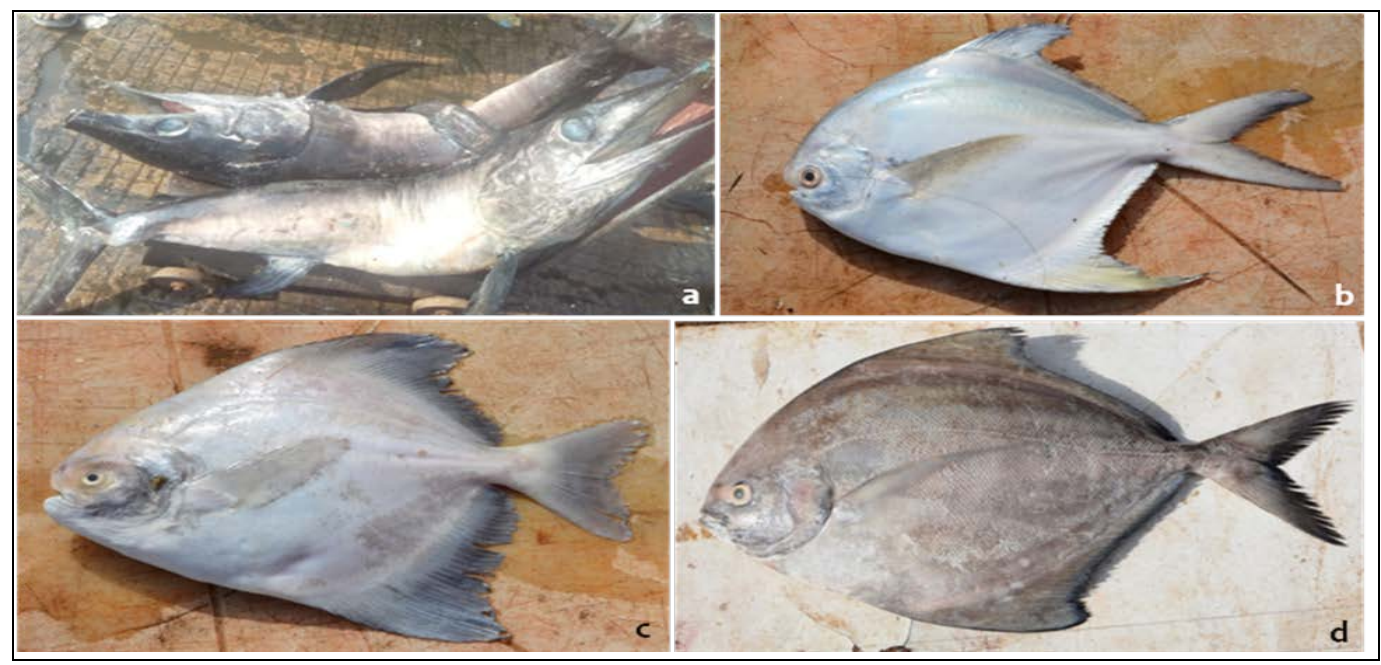

Figure 6a: Xiphias gladius, b. Pampus argenteus,

c. Pampus chinensis, d. Parastromateus niger.

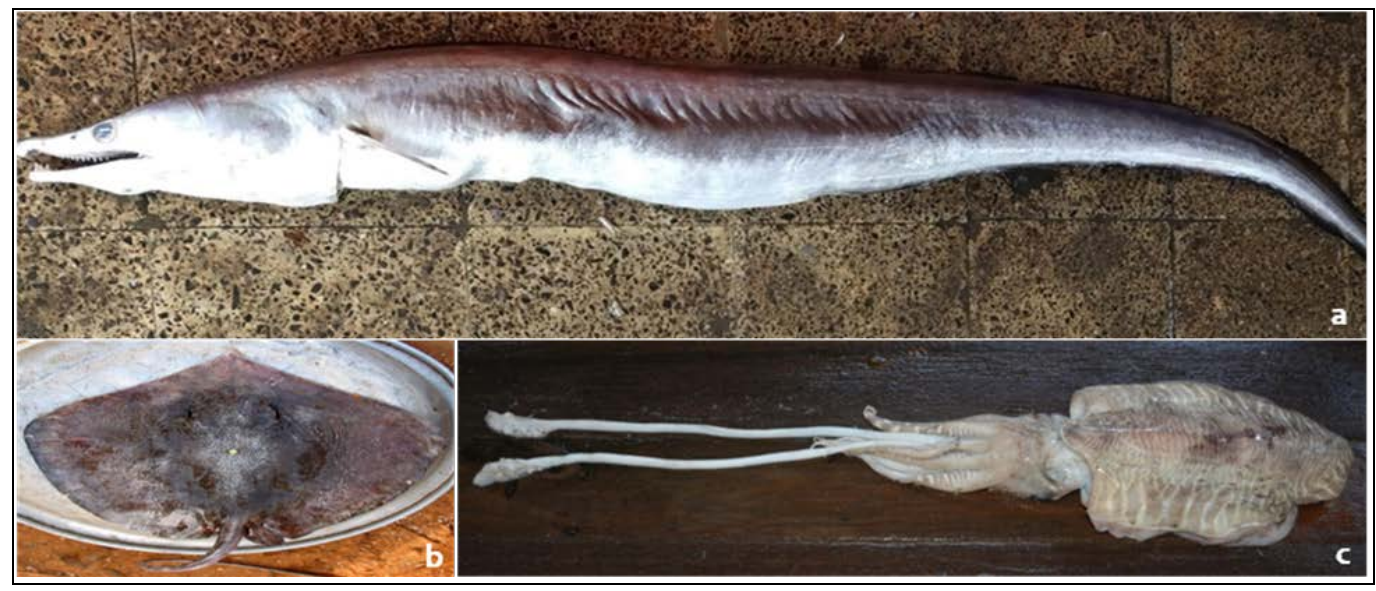

Figure 7: a. Muraenesox talabonoides,

b. Himantura bleekeri, c. Sepia pharaonis. 
According to IUCN Red List Species status globally, the twenty marine species belonged to four categories, Not Evaluated, Data Deficient, Least Concern and Near Threatened. Not evaluated (NE) indicates that IUCN has not yet assessed the species status. Data Deficient (DD) indicates that available information is not sufficient for a proper assessment of the conservation status of the species. Least Concern (LC) indicates that the species is not being a focus of species conservation and do not qualify as threatened, near threatened or conservation dependent. Near Threatened (NT) indicates that the species does not currently qualify for the threatened status but may be considered threatened with extinction in the near future. The NE category included Himantura bleekeri, Tachysurus thalassinus, Rostrelliger kanagurta, Pampus argenteus, P. chinensis, and Muraenesox talabonoides. The DD category included Scomberomorus guttatus, Makaira indica, and Sepia pharaonis. The LC category included Lutjanus argentimaculatus, Johnius dussumieri, Stolephorus commersonnii, Trichiurus lepturus, Lates calcarifer, Katsuwonus pelamis, Coryphaena hippurus, Xiphias gladius, and Parastromateus niger; the last two species populations have been stated to be decreasing globally. The NT category included Scomberomorus commerson and Thunnus albacares. 
Table 2: Common marine fin fish and shell-fish species landing at Visakhapatnam Fishing Harbour/local markets.

\begin{tabular}{|c|c|c|c|c|c|c|c|c|}
\hline $\begin{array}{l}\mathrm{S} . \\
\mathrm{N} \\
\mathrm{o} .\end{array}$ & Family & $\begin{array}{l}\text { Scientific } \\
\text { name }\end{array}$ & $\begin{array}{l}\text { Common } \\
\text { name }\end{array}$ & $\begin{array}{l}\text { Local } \\
\text { name }\end{array}$ & $\begin{array}{l}\text { Biotype } \\
\text { complex }\end{array}$ & $\begin{array}{c}\text { Feeding } \\
\text { habitat }\end{array}$ & $\begin{array}{c}\text { Fish } \\
\text { impor- } \\
\text { tance }\end{array}$ & $\begin{array}{l}\text { Approx. } \\
\text { price/kg }\end{array}$ \\
\hline \multicolumn{9}{|c|}{ Fin fishes } \\
\hline \multicolumn{9}{|c|}{ Elasmobranchs } \\
\hline 1 & $\begin{array}{l}\text { Dasya- } \\
\text { tidae }\end{array}$ & $\begin{array}{l}\text { Himantu- } \\
\text { ra } \\
\text { bleekeri } \\
\text { (Blyth, } \\
\text { 1860) }\end{array}$ & $\begin{array}{l}\text { Whiptail } \\
\text { sting ray }\end{array}$ & $\begin{array}{l}\text { Mullu } \\
\text { Tekku }\end{array}$ & $\begin{array}{l}\text { Marine } \\
\text { Brackish } \\
\text { Benthopelagic } \\
\text { Amphidro- } \\
\text { mous }\end{array}$ & $\begin{array}{l}\text { Carnivore } \\
\text { (small } \\
\text { crustaceans } \\
\text { bottom } \\
\text { living } \\
\text { invertebra- } \\
\text { tes) } \\
\end{array}$ & $\begin{array}{l}\text { Local } \\
\text { value }\end{array}$ & $\begin{array}{l}120 \\
\text { Indian } \\
\text { Rupee } \\
\text { (INR) } \\
1.63 \text { USD }\end{array}$ \\
\hline \multicolumn{9}{|c|}{ Teleosts } \\
\hline 2 & $\begin{array}{l}\text { Lutjani- } \\
\text { dae }\end{array}$ & $\begin{array}{l}\text { Lutjanus } \\
\text { argentima } \\
\text { culatus } \\
\text { (Forsskal, } \\
1775 \text { ) }\end{array}$ & $\begin{array}{l}\text { Mangrove } \\
\text { red } \\
\text { snapper }\end{array}$ & $\begin{array}{l}\text { Yerra } \\
\text { goraka/ } \\
\text { Ratigo- } \\
\text { raka }\end{array}$ & $\begin{array}{l}\text { Marine } \\
\text { Freshwater } \\
\text { Reef } \\
\text { Associated } \\
\text { Oceanodro- } \\
\text { mous } \\
\end{array}$ & $\begin{array}{l}\text { Carnivore } \\
\text { (fish } \\
\text { crustaceans) }\end{array}$ & $\begin{array}{l}\text { Local } \\
\text { value }\end{array}$ & $\begin{array}{l}480 \text { INR } \\
12 \text { USD }\end{array}$ \\
\hline 3 & $\begin{array}{l}\text { Sciae- } \\
\text { nidae }\end{array}$ & $\begin{array}{l}\text { Johnius } \\
\text { dussu- } \\
\text { mieri } \\
\text { (Cuvier, } \\
1830 \text { ) } \\
\end{array}$ & $\begin{array}{l}\text { Beard } \\
\text { croaker }\end{array}$ & $\begin{array}{l}\text { Geddam } \\
\text { gorssa }\end{array}$ & $\begin{array}{l}\text { Marine } \\
\text { Brackish } \\
\text { Demersal } \\
\text { Oceanodro- } \\
\text { mous } \\
\end{array}$ & $\begin{array}{l}\text { Carnivore } \\
\text { (invertebra- } \\
\text { tes, } \\
\text { small } \\
\text { fishes) } \\
\end{array}$ & $\begin{array}{l}\text { Local } \\
\text { value }\end{array}$ & $\begin{array}{l}\text { 150 INR } \\
\text { 2.0 USD }\end{array}$ \\
\hline 4 & Ariidae & $\begin{array}{l}\text { Tachysu- } \\
\text { rus } \\
\text { thalassic- } \\
\text { nus } \\
\text { (Ruppel, } \\
\text { 1837) }\end{array}$ & Cat Fish & Tella jella & $\begin{array}{l}\text { Demersal } \\
\text { Oceanodro- } \\
\text { mous }\end{array}$ & $\begin{array}{l}\text { Carnivore } \\
\text { (crustaceans } \\
\text { molluscs) }\end{array}$ & $\begin{array}{l}\text { Local } \\
\text { value }\end{array}$ & $\begin{array}{l}230 \text { INR } \\
3.14 \text { USD }\end{array}$ \\
\hline 5 & $\begin{array}{l}\text { Engrauli } \\
\text {-dae }\end{array}$ & $\begin{array}{l}\text { Stolepho- } \\
\text { rus } \\
\text { comer- } \\
\text { sonnii } \\
\text { (Lacepede, } \\
\text { 1803) } \\
\end{array}$ & $\begin{array}{l}\text { Commer- } \\
\text { sons } \\
\text { anchovy }\end{array}$ & Nethallu & $\begin{array}{l}\text { Marine } \\
\text { Brackish } \\
\text { Pelagic-neritic } \\
\text { Anadromous }\end{array}$ & $\begin{array}{l}\text { Carnivore } \\
\text { (surface } \\
\text { phyto- } \\
\text { plankton } \\
\text { prawn } \\
\text { larvae) } \\
\end{array}$ & $\begin{array}{l}\text { Local } \\
\text { value }\end{array}$ & $\begin{array}{l}200 \text { INR } \\
17 \text { USD }\end{array}$ \\
\hline 6 & $\begin{array}{l}\text { Trichiu- } \\
\text { ridae }\end{array}$ & $\begin{array}{l}\text { Trichiurus } \\
\text { lepturus } \\
\text { (Linnaeus, } \\
\text { 1758) }\end{array}$ & $\begin{array}{l}\text { Ribbon } \\
\text { Fish }\end{array}$ & $\begin{array}{l}\text { Pattisa- } \\
\text { vada }\end{array}$ & $\begin{array}{l}\text { Marine } \\
\text { Brackish } \\
\text { Benthopelagic } \\
\text { Amphidro- } \\
\text { mous } \\
\end{array}$ & $\begin{array}{l}\text { Carnivore } \\
\text { (surface } \\
\text { planktonic } \\
\text { crustaceans } \\
\text { small fishes) } \\
\end{array}$ & $\begin{array}{l}\text { Export } \\
\text { value }\end{array}$ & $\begin{array}{l}180 \text { INR } \\
12.8 \text { USD }\end{array}$ \\
\hline 7 & Latidae & $\begin{array}{l}\text { Lates } \\
\text { calcarifer } \\
\text { (Bloch, } \\
1790)\end{array}$ & $\begin{array}{l}\text { Giant sea } \\
\text { perch }\end{array}$ & $\begin{array}{l}\text { Pandu- } \\
\text { goppa/ } \\
\text { Pandu- } \\
\text { moyya }\end{array}$ & $\begin{array}{l}\text { Marine } \\
\text { Freshwater } \\
\text { Brackish } \\
\text { Demersal } \\
\text { Catadromous }\end{array}$ & $\begin{array}{l}\text { Carnivore } \\
\text { (fish } \\
\text { crustaceans) }\end{array}$ & $\begin{array}{l}\text { Export } \\
\text { value }\end{array}$ & $\begin{array}{l}450 \text { INR } \\
1.97 \text { USD }\end{array}$ \\
\hline 8 & $\begin{array}{l}\text { Scom- } \\
\text { bridae }\end{array}$ & $\begin{array}{l}\text { Rastre- } \\
\text { lliger } \\
\text { kanagurta } \\
\text { (Cuvier, } \\
1816 \text { ) }\end{array}$ & $\begin{array}{l}\text { Indian } \\
\text { mackerel }\end{array}$ & $\begin{array}{l}\text { Kanaga- } \\
\text { orta }\end{array}$ & $\begin{array}{l}\text { Marine } \\
\text { Pelagic-neritic } \\
\text { Oceano- } \\
\text { dromous }\end{array}$ & 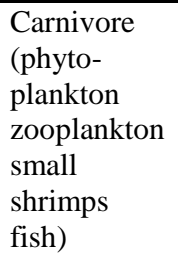 & $\begin{array}{l}\text { Export } \\
\text { value }\end{array}$ & $\begin{array}{l}200 \text { INR } \\
2.8 \text { USD }\end{array}$ \\
\hline
\end{tabular}


Table 2 (continued): Common marine fin fish and shell-fish species landing at Visakhapatnam Fishing Harbour/local markets.

\begin{tabular}{|c|c|c|c|c|c|c|c|c|}
\hline 9 & $\begin{array}{l}\text { Scom- } \\
\text { bridae }\end{array}$ & $\begin{array}{l}\text { Scombero } \\
\text { morus } \\
\text { commer- } \\
\text { son } \\
\text { (Lacepede, } \\
\text { 1800) }\end{array}$ & $\begin{array}{l}\text { Narrow } \\
\text { barred } \\
\text { seerfish }\end{array}$ & Konemu & $\begin{array}{l}\text { Marine } \\
\text { Pelagic-neritic } \\
\text { Oceano- } \\
\text { dromous }\end{array}$ & $\begin{array}{l}\text { Carnivore } \\
\text { (small fish) }\end{array}$ & $\begin{array}{l}\text { Export } \\
\text { value }\end{array}$ & $\begin{array}{l}900 \text { INR } \\
12.29\end{array}$ \\
\hline 10 & $\begin{array}{l}\text { Scom- } \\
\text { bridae }\end{array}$ & $\begin{array}{l}\text { Scombe- } \\
\text { romorus } \\
\text { guttatus } \\
\text { (Bloch } \\
\text { and } \\
\text { Schneider, } \\
1801 \text { ) } \\
\end{array}$ & $\begin{array}{l}\text { Indo } \\
\text { pacific } \\
\text { seer fish }\end{array}$ & $\begin{array}{l}\text { Vanja- } \\
\text { ramu }\end{array}$ & $\begin{array}{l}\text { Marine } \\
\text { Brackish } \\
\text { Pelagic-neritic } \\
\text { Oceano- } \\
\text { dromous }\end{array}$ & $\begin{array}{l}\text { Carnivore } \\
\text { (small fish, } \\
\text { squids, } \\
\text { crustaceans) }\end{array}$ & $\begin{array}{l}\text { Export } \\
\text { value }\end{array}$ & $\begin{array}{l}650 \text { INR } \\
\text { 8.87 USD }\end{array}$ \\
\hline 11 & $\begin{array}{l}\text { Scom- } \\
\text { bridae }\end{array}$ & $\begin{array}{l}\text { Katsu- } \\
\text { wonus } \\
\text { pelamis } \\
\text { (Linnaeus, } \\
1758 \text { ) }\end{array}$ & $\begin{array}{l}\text { Skip Jack } \\
\text { Tuna }\end{array}$ & $\begin{array}{l}\text { Namala } \\
\text { soora }\end{array}$ & $\begin{array}{l}\text { Marine } \\
\text { Pelagic- } \\
\text { oceanic } \\
\text { Oceano- } \\
\text { dromous }\end{array}$ & $\begin{array}{l}\text { Carnivore } \\
\text { (fish, } \\
\text { crustaceans, } \\
\text { molluscs } \\
\text { cephalopods }\end{array}$ & $\begin{array}{l}\text { Export } \\
\text { value }\end{array}$ & $\begin{array}{l}160 \text { INR } \\
2.18 \text { USD }\end{array}$ \\
\hline 12 & $\begin{array}{l}\text { Scom- } \\
\text { bridae }\end{array}$ & $\begin{array}{l}\text { Thunnus } \\
\text { albacares } \\
\text { (Bonna- } \\
\text { terre } \\
1788 \text { ) }\end{array}$ & $\begin{array}{l}\text { Yellow } \\
\text { Fin Tuna }\end{array}$ & $\begin{array}{l}\text { Recca } \\
\text { soora }\end{array}$ & $\begin{array}{l}\text { Marin } \\
\text { Brackish } \\
\text { Pelagic- } \\
\text { oceanic } \\
\text { Oceano- } \\
\text { dromous } \\
\end{array}$ & $\begin{array}{l}\text { Carnivore } \\
\text { (fish, } \\
\text { crustaceans, } \\
\text { squids) }\end{array}$ & $\begin{array}{l}\text { Export } \\
\text { value }\end{array}$ & $\begin{array}{l}200 \text { INR } \\
2.73 \text { USD }\end{array}$ \\
\hline 13 & $\begin{array}{l}\text { Corypha } \\
\text {-enidae }\end{array}$ & $\begin{array}{l}\text { Corypha- } \\
\text { ena } \\
\text { hippurus } \\
\text { (Linnaeus, } \\
1758 \text { ) }\end{array}$ & $\begin{array}{l}\text { Mahi } \\
\text { Mahi }\end{array}$ & $\begin{array}{l}\text { Abhilasha } \\
\text { cheppa }\end{array}$ & $\begin{array}{l}\text { Marine } \\
\text { Brackish } \\
\text { Pelagic-neritic } \\
\text { Oceano- } \\
\text { dromous } \\
\end{array}$ & $\begin{array}{l}\text { Carnivore } \\
\text { (zoo- } \\
\text { plankton, } \\
\text { fishes, } \\
\text { crustaceans) } \\
\end{array}$ & $\begin{array}{l}\text { Export } \\
\text { value }\end{array}$ & $\begin{array}{l}290 \text { INR } \\
3.96 \text { USD }\end{array}$ \\
\hline 14 & $\begin{array}{l}\text { Istio- } \\
\text { phoridae }\end{array}$ & $\begin{array}{l}\text { Makaira } \\
\text { indica } \\
\text { (Cuvier, } \\
\text { 1832) }\end{array}$ & $\begin{array}{l}\text { Black } \\
\text { Marlin }\end{array}$ & $\begin{array}{l}\text { Nalla } \\
\text { kommu } \\
\text { konemu }\end{array}$ & $\begin{array}{l}\text { Marine } \\
\text { Pelagic- } \\
\text { oceanic } \\
\text { Oceano- } \\
\text { dromous }\end{array}$ & $\begin{array}{l}\text { Carnivore } \\
\text { (small fish, } \\
\text { crustaceans, } \\
\text { cephalo- } \\
\text { pods) }\end{array}$ & $\begin{array}{l}\text { Export } \\
\text { value }\end{array}$ & $\begin{array}{l}260 \text { INR } \\
3.55 \text { USD }\end{array}$ \\
\hline 15 & $\begin{array}{l}\text { Xiphii- } \\
\text { dae }\end{array}$ & $\begin{array}{l}\text { Xiphias } \\
\text { gladius } \\
\text { (Linnaeus, } \\
1758 \text { ) }\end{array}$ & $\begin{array}{l}\text { Sword } \\
\text { fish }\end{array}$ & $\begin{array}{l}\text { Kommu } \\
\text { konemu }\end{array}$ & $\begin{array}{l}\text { Marine } \\
\text { Pelagic- } \\
\text { oceanic } \\
\text { Oceano- } \\
\text { dromous } \\
\end{array}$ & $\begin{array}{l}\text { Carnivore } \\
\text { (small fish, } \\
\text { crustaceans, } \\
\text { squids) }\end{array}$ & $\begin{array}{l}\text { Export } \\
\text { value }\end{array}$ & $\begin{array}{l}280 \text { INR } \\
3.82 \text { USD }\end{array}$ \\
\hline 16 & $\begin{array}{l}\text { Stroma- } \\
\text { teidae }\end{array}$ & $\begin{array}{l}\text { Pampus } \\
\text { argenteus } \\
\text { (Euphra- } \\
\text { sen, 1788) }\end{array}$ & $\begin{array}{l}\text { Silver } \\
\text { pomfret }\end{array}$ & $\begin{array}{l}\text { Tella } \\
\text { chanduva }\end{array}$ & $\begin{array}{l}\text { Marine } \\
\text { Benthopelagic } \\
\text { Oceano- } \\
\text { dromous }\end{array}$ & $\begin{array}{l}\text { In-shore } \\
\text { species } \\
\text { (fish eggs, } \\
\text { ctenophores, } \\
\text { salps, } \\
\text { medusa, } \\
\text { zoo- } \\
\text { plankton) }\end{array}$ & $\begin{array}{l}\text { Export } \\
\text { value }\end{array}$ & $\begin{array}{l}700 \text { INR } \\
9.56 \text { USD }\end{array}$ \\
\hline 17 & $\begin{array}{l}\text { Stroma- } \\
\text { teidae }\end{array}$ & $\begin{array}{l}\text { Pampus } \\
\text { chinensis } \\
\text { (Euphra- } \\
\text { sen, 1788) }\end{array}$ & $\begin{array}{l}\text { Chinese } \\
\text { pomfret }\end{array}$ & $\begin{array}{l}\text { Attukoyya } \\
\text { / } \\
\text { Atukula } \\
\text { chanduva }\end{array}$ & $\begin{array}{l}\text { Marine } \\
\text { Brackish } \\
\text { Benthopelagic } \\
\text { Amphidro- } \\
\text { mous }\end{array}$ & $\begin{array}{l}\text { Carnivore } \\
\text { (fish eggs, } \\
\text { ctenophores } \\
\text { salps } \\
\text { medusa zoo- } \\
\text { plankton) }\end{array}$ & $\begin{array}{l}\text { Local } \\
\text { value }\end{array}$ & $\begin{array}{l}500 \text { INR } \\
6.83 \text { USD }\end{array}$ \\
\hline
\end{tabular}


Table 2 (continued): Common marine fin fish and shell-fish species landing at Visakhapatnam Fishing Harbour/local markets.

\begin{tabular}{|c|l|l|l|l|l|l|l|l|}
\hline 18 & $\begin{array}{l}\text { Caran- } \\
\text { gidae }\end{array}$ & $\begin{array}{l}\text { Parastrom } \\
\text { ateus } \\
\text { niger } \\
\text { (Bloch, } \\
1795)\end{array}$ & $\begin{array}{l}\text { Black } \\
\text { pomfret } \\
\text { chanduva }\end{array}$ & $\begin{array}{l}\text { Marine } \\
\text { Brackish } \\
\text { Reef } \\
\text { associated } \\
\text { Amphidro- } \\
\text { mous }\end{array}$ & $\begin{array}{l}\text { Carnivore } \\
\text { (fish eggs, } \\
\text { ctenophores, } \\
\text { salps, } \\
\text { medusa, } \\
\text { zoo- } \\
\text { plankton) }\end{array}$ & $\begin{array}{l}\text { Local } \\
\text { value }\end{array}$ & $\begin{array}{l}\text { 280 INR } \\
\text { 3.81 USD }\end{array}$ \\
\hline 19 & $\begin{array}{l}\text { Muraen } \\
\text { esocidae }\end{array}$ & $\begin{array}{l}\text { Murae- } \\
\text { nesox } \\
\text { talabo- } \\
\text { noides } \\
\text { (Bleeker, } \\
1853)\end{array}$ & $\begin{array}{l}\text { Indian } \\
\text { pike } \\
\text { conger }\end{array}$ & $\begin{array}{l}\text { Tella } \\
\text { Pamu } \\
\text { cheppa }\end{array}$ & $\begin{array}{l}\text { Marine } \\
\text { Brackish } \\
\text { Bathy- } \\
\text { demersal } \\
\text { Amphidro- } \\
\text { mous }\end{array}$ & $\begin{array}{l}\text { Carnivore } \\
\text { (bottom } \\
\text { fish, } \\
\text { crustaceans) }\end{array}$ & $\begin{array}{l}\text { Local } \\
\text { value }\end{array}$ & 1 130 INR \\
1.77 USD \\
\hline Shell fishes
\end{tabular}

Spawning migration classification of common marine fin- and shell-fish species landing/sold at Fishing Harbour and local fish markets of Visakhapatnam: These marine species were classified based on spawning migration into anadromous, catadromous, amphidromous and oceanodromous. Anadromous and catadromous types included one species each, the former was represented by Stolephorus commersonnii while the latter was represented by Lates calcarifer. Amphidromous type included five species, Himantura bleekeri, Trichiurus lepturus, Pampus chinensis, Parastromateus niger, and Muraenesox talabonoides. Oceanodromous type included 13 species which included Lutjanus argentimaculatus, Johnius dussumieri, Tachysurus thalassinus, Rastrelliger kanagurta, Scomberomorus commerson, S. guttatus, Katsuwonus pelamis, Thunnus albacares, Coryphaena hippurus, Makaira indica, Xiphias gladius, Pampus argenteus, and Sepia pharaonis (Tab. 2; Fig. 1).

Biotype complex, feeding habit, local and export value, and price value of common marine fin- and shell-fish species landing/sold at Fishing Harbour and local fish markets of Visakhapatnam: All twenty marine species are carnivores and the food items vary with each species. The food items included crustaceans, molluscs, fish eggs, small fish, phytoplanktons, and zooplanktons. The fish species such as Trichiurus lepturus, Lates calcarifer, Rastrelliger kanagurta, Scomberomorus commerson, S. guttatus, Katsuwonus pelamis, Thunnus albacares, Coryphaena hippurus, Makaira indica, Xiphias gladius, and Pampus argenteus, and the cephalopod molluscan, Sepia pharaonis were found to be of export value while all other species were found to be of local value and they are regularly purchased by locals (Figs. 8a-c). The price value of each species in local currency as well as U.S. Dollar currency is recorded in table 2 . 


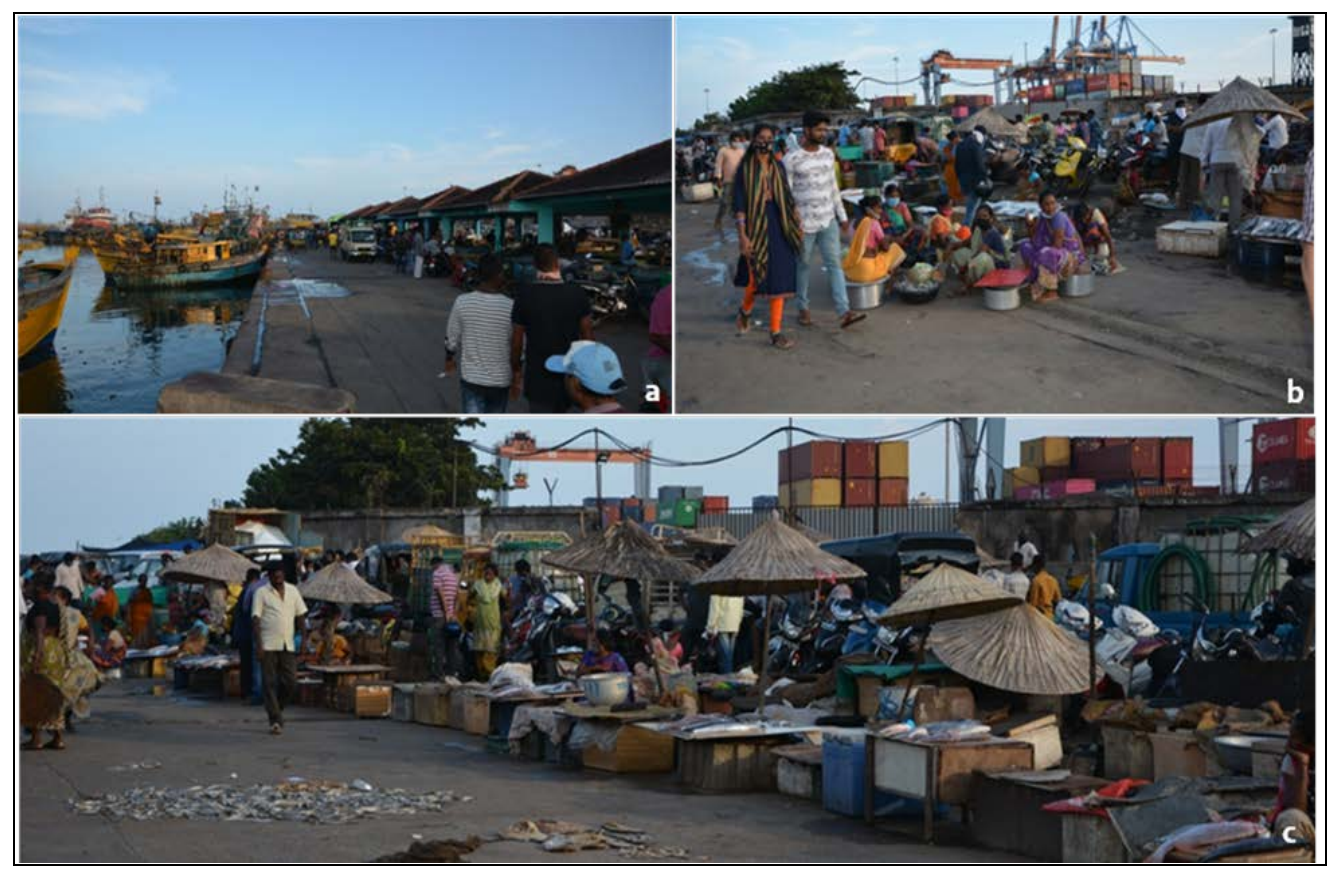

Figure 8a: Fish vendors and consumers at the market Visakhapatnam market; 8b, c Visakhapatnam Fishing Harbour market.

The fishermen use sun-drying methods involving fish drying on the boat roof top (Fig. 9a), fish drying by hanging (Fig. 9b) and fish drying on the floor (Fig. 9c) of the fishing harbour market. The dried fish are loaded into bamboo baskets and transported to local markets where fish vendors buy and sell them as a regular livelihood source (Figs. 10a-c).

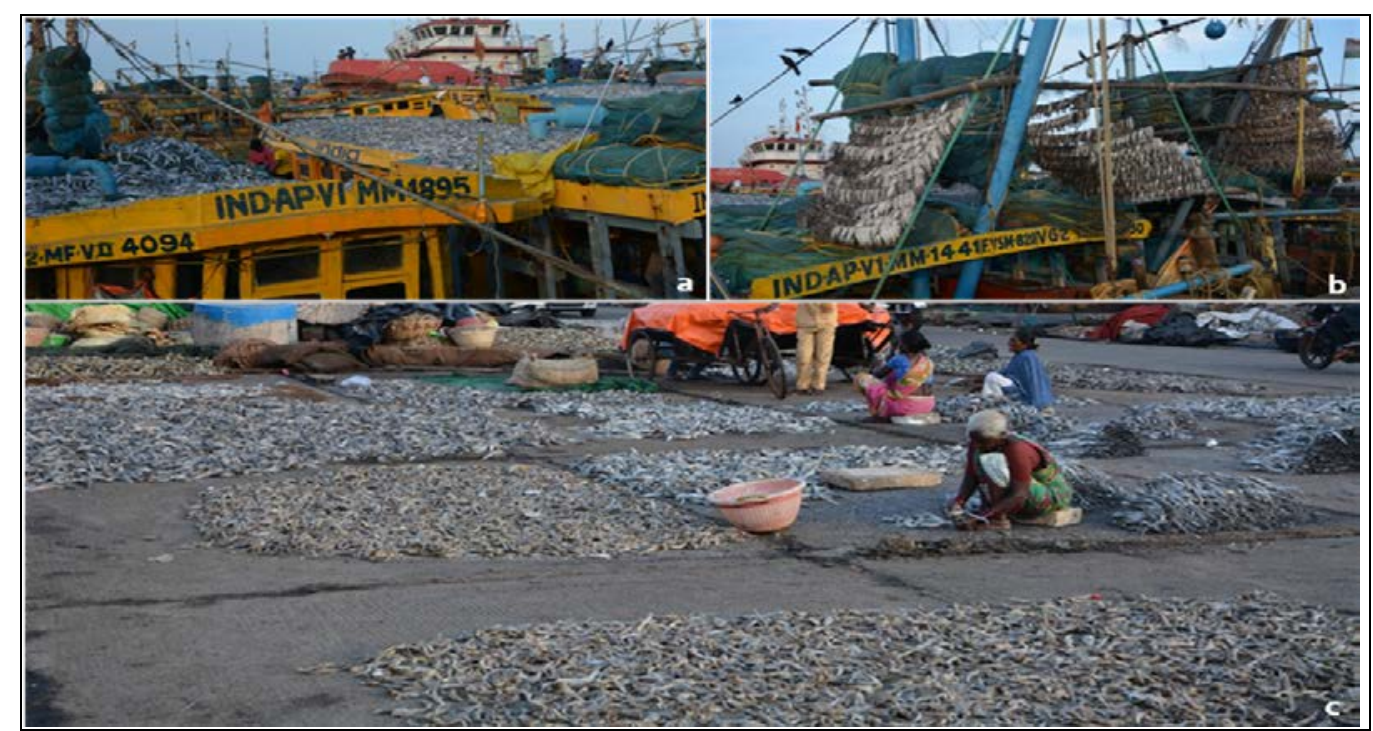

Figure 9: Visakhapatnam Fishing Harbour - sun-drying method -

a. Drying on the boat roof top, b. Drying by hanging fish, c. Drying on the floor. 


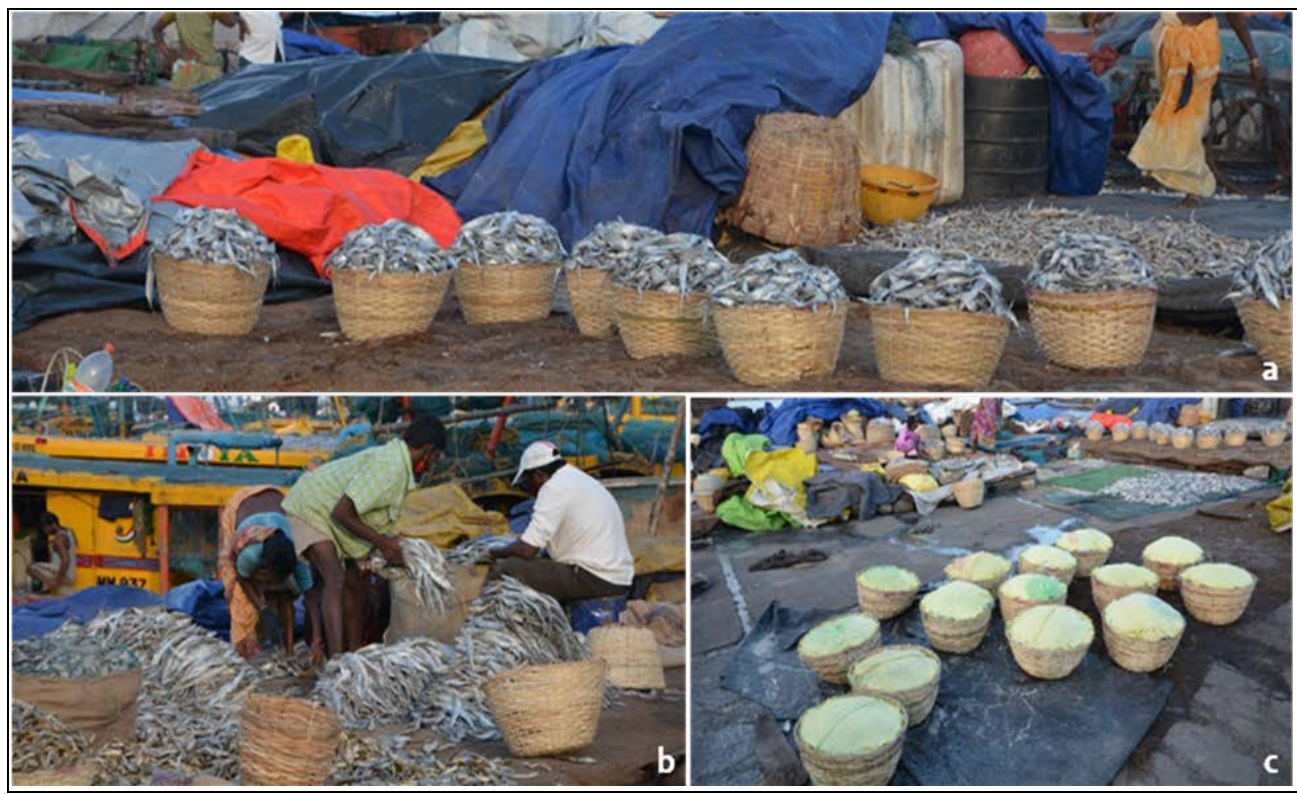

Figure 10: Visakhapatnam fishing harbour - a. Bamboo baskets loaded with dried fish, b. Dried fish being loaded into bamboo baskets,

c. Bamboo baskets loaded with dried fish ready for transport to local markets.

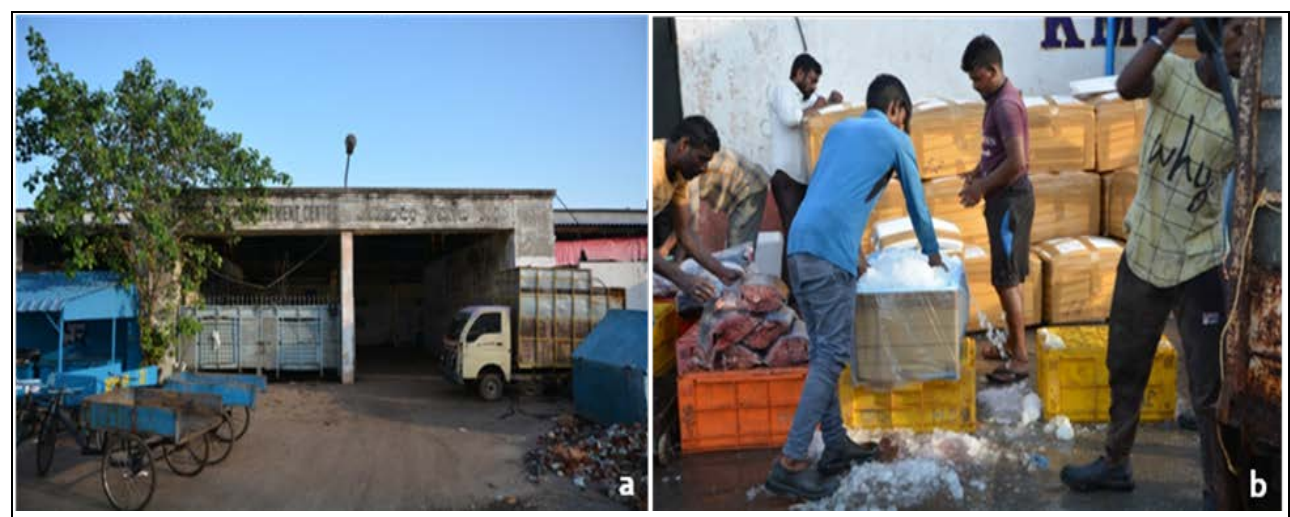

Figure 11: a. Seafood Export Trading Centre, Visakhapatnam, b. Fish packing being done for export.

Six local companies are involved in exporting edible marine fish and molluscan species to other countries through Visakhapatnam Sea Food Export Trade Center (VSFETC) (Figs. 11a, b). The companies are SMSEA Corporation, SVR Sea Food Private Limited, Ghan Marine Products, Global Marine Products, APS Sea Foods and Southern Sea Foods Exports. The fish species exported through VSFETC are Trichiurus lepturus, Katsuwonus pelamis, Thunnus albacares, Makaira indica, Xiphias gladius, and Rastrelliger kanagurta. The crustacean penaeids exported through VSFETC are Penaeus monodon, P. indicus, $P$. semisulcatus, and Pandalus borealis. In molluscans, the cephalod species, Sepia pharaonis is the only species exported through VSFETC. The fish species, Lates calcarifer, Scomberomorus commerson, S. guttatus, Coryphaena hippurus, and Pampus argenteus despite their export value are not being exported due to heavy consumption locally (Tab. 3). 
Table 3: Marine species exported through Visakhapatnam Sea Food Export Trade Center.

\begin{tabular}{|c|c|c|c|}
\hline \multicolumn{4}{|c|}{ SMSEA Corporation } \\
\hline Species & $\begin{array}{c}\mathrm{N} \\
\text { (days) }\end{array}$ & $\begin{array}{c}\text { Range } \\
\text { (kg) }\end{array}$ & $\begin{array}{c}\mathrm{M} \pm \mathrm{SD} \\
(\mathrm{kg})\end{array}$ \\
\hline Sepia pharaonis & 15 & $4,045.90$ & $1,512.21 \pm 985.51$ \\
\hline Trichiurus lepturus & 15 & $8,089.66$ & $1,737.36 \pm 2,258.91$ \\
\hline Katsuwonus pelamis & 15 & $6,350.29$ & $1,657.50 \pm 2,181.74$ \\
\hline Thunnus albacares & 15 & $6,350.29$ & $846.23 \pm 1,703.94$ \\
\hline Makaira indica & 15 & $2,431.26$ & $175.42 \pm 626.18$ \\
\hline Xiphias gladius & 15 & $6,350.29$ & $638.12 \pm 1,729.47$ \\
\hline \multicolumn{4}{|c|}{ SVR Sea Food Private Limited } \\
\hline Sepia pharaonis & 15 & $3,628.74$ & $1,059.90 \pm 1050.47$ \\
\hline Trichiurus lepturus & 15 & $2,267.96$ & $686.74 \pm 704.52$ \\
\hline Penaeus indicus & 15 & $2,267.96$ & $838.89 \pm 845.73$ \\
\hline Pandalus borealis & 15 & 907.19 & $241.81 \pm 288.26$ \\
\hline \multicolumn{4}{|c|}{ Ghan Marine Products } \\
\hline Sepia pharaonis & 15 & 410.00 & $134.00 \pm 144.46$ \\
\hline Trichiurus lepturus & 15 & 300.00 & $45.87 \pm 92.97$ \\
\hline Katsuwonus pelamis & 15 & $2,540.12$ & $730.08 \pm 998.80$ \\
\hline Thunnus albacares & 15 & 300.00 & $26.67 \pm 79.88$ \\
\hline \multicolumn{4}{|c|}{ Global Marine Exports } \\
\hline Pandalus borealis & 26 & $9,932.62$ & $2,883.92 \pm 2,128.76$ \\
\hline \multicolumn{4}{|c|}{ APS Sea Foods } \\
\hline Sepia pharaonis & 15 & 684 & $591 \pm 195$ \\
\hline Trichiurus lepturus & 15 & 2,500 & $604 \pm 743$ \\
\hline Katsuwonus pelamis & 15 & 6,700 & $2,007 \pm 2,118$ \\
\hline Thunnus albacares & 15 & 2,500 & $856 \pm 957$ \\
\hline Makaira indica & 15 & 86 & $11 \pm 26$ \\
\hline Xiphias gladius & 15 & 50 & $8 \pm 17$ \\
\hline Rastrelliger kanagurta & 15 & 180 & $28 \pm 59$ \\
\hline \multicolumn{4}{|c|}{ Southern Sea Foods Exports } \\
\hline Pandalus borealis & 15 & 578 & $324 \pm 152$ \\
\hline Penaeus monodon & 15 & 90 & $70 \pm 24$ \\
\hline Penaeus indicus & 15 & 240 & $67 \pm 56$ \\
\hline Penaeus semisulcatus & 15 & 75 & $36 \pm 17$ \\
\hline
\end{tabular}


Gears used for capturing common marine fin- and shell-fish species landing/sold at Fishing Harbour and local fish markets of Visakhapatnam: Gears used for capturing the common marine species landing at Fishing Harbour and local markets of Visakhapatnam included eight categories mentioned in the International Standard Statistical Classification of Fishing Gear (ISSCFG, 2016). The categories were Surrounding nets, Seine nets, Trawls, Lift nets, Gillnets, Entangling nets, Traps, Hooks, Lines, and Miscellaneous Gear. The Surrounding Net Gear category was represented by Purse seine; Seine net Gear category by boat seines, seine nets, and beach seines; Trawls category by trawl nets and bottom trawls; Lift nets category by lift nets; Gillnets and Entangling nets category by gillnets, drift gillnets and set gillnets; Traps category by Bamboo skate traps and traps; Hooks and Lines category by hand lines, long lines, trolling lines, pole and lines; and Miscellaneous gear category (not classified with the above groups) by bag nets, dip nets, and harpoons. The Gears used varied with the fish species and the gear types are recorded in table 4. In the same table, the period of occurrence, depth of occurrence (in meters), size at maturity and spawning season are also noted. Xiphias gladius is the only species that occurs commonly throughout the year while all other species occur from three to 10 months. All twenty marine species occur at different depths and the depth of occurrence ranged from 0 to 2,870 m. Among these species, Xiphias gladius is the largest in size at maturity. The size of other fish/molluscan species at maturity varied from 7 to $250 \mathrm{~cm}$. Lutjanus argentimaculatus, Johnius dussumieri, Trichurus lepturus, Katsuwonus pelamis, and Xiphias gladius spawn throughout the year while all other species are seasonal in spawning.

Description of morphometrics of common marine fin- and shell-fish species landing/sold at Fishing Harbour and local fish markets of Visakhapatnam: The standard length, total length, depth of body, head length (in $\mathrm{cm}$ ) and weight (in g) were recorded for teleosts, Lutjanus argentimaculatus, Johnius dussumieri, Tachysurus thalassinus, Stolephorus commersonii, Trichiurus lepturus, Lates calcarifer, Rastrelliger kanagurta, Scomberomorus commerson, S. guttatus, Katsuwonus pelamis, Thunnus albacares, Coryphaena hippurus, Makaira indica, Xiphias gladius, Pampus argenteus, P. chinensis, Parastromateus niger, and Muraenesox talabonoides. Disc length, total length (in $\mathrm{cm}$ ) and weight (in g) were recorded for the elasmobranch, Himantura bleekeri while mantle length, total length (in $\mathrm{cm}$ ) and weight (in g) were recorded for cephalod molluscan, Sepia pharaonis (Tabs. 5 and 7). 
Table 4: Gears used for fish capture, peak period of occurrence, depth of occurrence, size at maturity and spawning season of most common marine fin fish and shell-fish species landing at Visakhapatnam Fishing Harbour.

\begin{tabular}{|c|c|c|c|c|c|c|}
\hline $\begin{array}{l}\text { Sp. } \\
\text { no. }\end{array}$ & Species & $\begin{array}{l}\text { Gears } \\
\text { used for } \\
\text { capturing }\end{array}$ & $\begin{array}{c}\text { Peak } \\
\text { period of } \\
\text { occurrence }\end{array}$ & $\begin{array}{l}\text { Depth of } \\
\text { occurrence } \\
\text { (range } \\
\text { in m) }\end{array}$ & $\begin{array}{l}\text { Size at } \\
\text { maturity } \\
(\mathrm{cm})\end{array}$ & $\begin{array}{c}\text { Spawning } \\
\text { season }\end{array}$ \\
\hline 1. & $\begin{array}{l}\text { Lutjanus } \\
\text { argentimaculatus }\end{array}$ & $\begin{array}{l}\text { Hand lines } \\
\text { Long lines } \\
\text { Trawl nets }\end{array}$ & $\begin{array}{l}\text { September- } \\
\text { January }\end{array}$ & $1-12$ & $50-57$ & Year-long \\
\hline 2. & $\begin{array}{l}\text { Johnius } \\
\text { dussumieri }\end{array}$ & $\begin{array}{l}\text { Trawl net } \\
\text { Boat seines }\end{array}$ & $\begin{array}{l}\text { October- } \\
\text { March }\end{array}$ & $30-70$ & $11-16.5$ & Year-long \\
\hline 3. & $\begin{array}{l}\text { Tachysurus } \\
\text { thalassinus }\end{array}$ & $\begin{array}{l}\text { Skate traps } \\
\text { Bag nets } \\
\text { Dip nets }\end{array}$ & $\begin{array}{l}\text { March- } \\
\text { June } \\
\text { September- } \\
\text { October } \\
\end{array}$ & $10-80$ & $33-45$ & April-August \\
\hline 4. & $\begin{array}{l}\text { Stolephorus } \\
\text { commersonnii }\end{array}$ & $\begin{array}{l}\text { Skate nets } \\
\text { Boat seines } \\
\text { Seine nets }\end{array}$ & $\begin{array}{l}\text { October- } \\
\text { April }\end{array}$ & $0-50$ & $7-7.3$ & March-May \\
\hline 5. & $\begin{array}{l}\text { Trichiurus } \\
\text { lepturus }\end{array}$ & $\begin{array}{l}\text { Trawl nets } \\
\text { Bag nets }\end{array}$ & $\begin{array}{l}\text { July- } \\
\text { April }\end{array}$ & $100-350$ & $63.9-69.3$ & Year-long \\
\hline 6. & $\begin{array}{l}\text { Lates } \\
\text { calcarifer }\end{array}$ & $\begin{array}{l}\text { Trawl nets } \\
\text { Gill nets } \\
\text { Seine nets } \\
\text { Hand lines } \\
\text { Traps }\end{array}$ & $\begin{array}{l}\text { August- } \\
\text { February }\end{array}$ & $10-40$ & $29-60$ & $\begin{array}{l}\text { February- } \\
\text { March } \\
\text { June-August } \\
\text { October- } \\
\text { December }\end{array}$ \\
\hline 7. & $\begin{array}{l}\text { Rastrelliger } \\
\text { kanagurta }\end{array}$ & $\begin{array}{l}\text { Beach } \\
\text { seines Drift } \\
\text { gillnets } \\
\text { Purse } \\
\text { seines } \\
\text { Trolling } \\
\text { nets } \\
\end{array}$ & $\begin{array}{l}\text { August- } \\
\text { November }\end{array}$ & $20-90$ & $20-24.5$ & $\begin{array}{l}\text { January- } \\
\text { March } \\
\text { August- } \\
\text { October }\end{array}$ \\
\hline 8. & $\begin{array}{l}\text { Scomberomorus } \\
\text { commerson }\end{array}$ & $\begin{array}{l}\text { Drift } \\
\text { gillnets } \\
\text { Trolling } \\
\text { lines Hand } \\
\text { lines }\end{array}$ & $\begin{array}{l}\text { October- } \\
\text { December }\end{array}$ & $10-70$ & $55-82$ & $\begin{array}{l}\text { January- } \\
\text { September }\end{array}$ \\
\hline 9. & $\begin{array}{l}\text { Scomberomorus } \\
\text { guttatus }\end{array}$ & $\begin{array}{l}\text { Drift } \\
\text { gillnets } \\
\text { Trawl nets } \\
\text { Purse } \\
\text { seines } \\
\text { Trolling } \\
\text { nets }\end{array}$ & $\begin{array}{l}\text { October- } \\
\text { December }\end{array}$ & $15-200$ & $48-52$ & $\begin{array}{l}\text { January- } \\
\text { August } \\
\text { April-May }\end{array}$ \\
\hline
\end{tabular}


Table 4 (continued): Gears used for fish capture, peak period of occurrence, depth of occurrence, size at maturity and spawning season of most common marine fin fish and shellfish species landing at Visakhapatnam Fishing Harbour.

\begin{tabular}{|c|c|c|c|c|c|c|}
\hline $\begin{array}{l}\text { Sp. } \\
\text { no. }\end{array}$ & Species & $\begin{array}{l}\text { Gears used } \\
\text { for capturing }\end{array}$ & $\begin{array}{l}\text { Peak period } \\
\text { of } \\
\text { occurrence }\end{array}$ & $\begin{array}{l}\text { Depth of } \\
\text { occurrence } \\
\text { (range } \\
\text { in m) }\end{array}$ & $\begin{array}{c}\text { Size at } \\
\text { maturity } \\
(\mathrm{cm})\end{array}$ & $\begin{array}{l}\text { Spawning } \\
\text { season }\end{array}$ \\
\hline 10. & $\begin{array}{l}\text { Katsuwonus } \\
\text { pelamis }\end{array}$ & $\begin{array}{l}\text { Purse seines } \\
\text { Gill nets } \\
\text { Bamboo } \\
\text { skate Traps } \\
\text { Lift nets } \\
\end{array}$ & $\begin{array}{l}\text { October- } \\
\text { May }\end{array}$ & $0-260$ & $40-45$ & Year-long \\
\hline 11. & $\begin{array}{l}\text { Thunnus } \\
\text { albacares }\end{array}$ & $\begin{array}{l}\text { Purse seines } \\
\text { Pole and } \\
\text { lines } \\
\text { Trolling nets } \\
\text { Gill nets }\end{array}$ & $\begin{array}{l}\text { October- } \\
\text { January }\end{array}$ & $1-250$ & $78-158$ & March-May \\
\hline 12. & $\begin{array}{l}\text { Coryphaena } \\
\text { hippurus }\end{array}$ & $\begin{array}{l}\text { Trolling nets } \\
\text { Long lines } \\
\text { Purse seines } \\
\text { Drift gillnets } \\
\end{array}$ & $\begin{array}{l}\text { June- } \\
\text { October }\end{array}$ & $0-85$ & $35-93.1$ & $\begin{array}{l}\text { March- } \\
\text { September }\end{array}$ \\
\hline 13. & $\begin{array}{l}\text { Makaira } \\
\text { indica }\end{array}$ & $\begin{array}{l}\text { Long lines } \\
\text { Set gillnets }\end{array}$ & $\begin{array}{l}\text { March- } \\
\text { September }\end{array}$ & $0-915$ & - & March-June \\
\hline 14. & $\begin{array}{l}\text { Xiphias } \\
\text { gladius }\end{array}$ & $\begin{array}{l}\text { Long lines } \\
\text { Trolling nets }\end{array}$ & $\begin{array}{l}\text { Throughout } \\
\text { the year }\end{array}$ & $0-2870$ & $156-250$ & $\begin{array}{l}\text { Year-long } \\
\text { Peak: March- } \\
\text { June }\end{array}$ \\
\hline 15. & $\begin{array}{l}\text { Pampus } \\
\text { argenteus }\end{array}$ & $\begin{array}{l}\text { Trawl nets } \\
\text { Gillnets }\end{array}$ & $\begin{array}{l}\text { August- } \\
\text { January }\end{array}$ & $\begin{array}{c}\text { Inshore } \\
5-80 \\
\end{array}$ & $18-23.5$ & January-June \\
\hline 16. & $\begin{array}{l}\text { Pampus } \\
\text { chinensis }\end{array}$ & $\begin{array}{l}\text { Trawl nets } \\
\text { Gill nets }\end{array}$ & $\begin{array}{l}\text { August- } \\
\text { December }\end{array}$ & $0-80$ & $22.5-26.5$ & January-May \\
\hline 17. & $\begin{array}{l}\text { Parastromateus } \\
\text { niger }\end{array}$ & $\begin{array}{l}\text { Trawl nets } \\
\text { Gill nets }\end{array}$ & $\begin{array}{l}\text { August- } \\
\text { December }\end{array}$ & $15-40$ & $22-24$ & $\begin{array}{l}\text { February- } \\
\text { April }\end{array}$ \\
\hline 18. & $\begin{array}{l}\text { Muraenesox } \\
\text { talabonoides }\end{array}$ & $\begin{array}{l}\text { Long lines } \\
\text { Trawl nets } \\
\text { Drift nets } \\
\end{array}$ & $\begin{array}{l}\text { July- } \\
\text { September }\end{array}$ & $800-875$ & $122-125$ & $\begin{array}{l}\text { April-May } \\
\text { September- } \\
\text { October } \\
\end{array}$ \\
\hline 19. & $\begin{array}{l}\text { Himantura } \\
\text { bleekeri }\end{array}$ & $\begin{array}{l}\text { Long lines } \\
\text { Harpoons } \\
\text { Bottom } \\
\text { trawls } \\
\text { Gill nets } \\
\end{array}$ & $\begin{array}{l}\text { December- } \\
\text { May }\end{array}$ & $30-40$ & - & Ovoviviparous \\
\hline 20. & $\begin{array}{l}\text { Sepia } \\
\text { pharaonis }\end{array}$ & Trawl nets & $\begin{array}{l}\text { September- } \\
\text { January }\end{array}$ & $0-130$ & $12-24.4$ & $\begin{array}{l}\text { October- } \\
\text { November } \\
\text { February- } \\
\text { March } \\
\end{array}$ \\
\hline
\end{tabular}


Table 5: Morphometric characteristics of common marine fin fish and shell-fish species landing at Visakhapatnam Fishing Harbour.

\begin{tabular}{|c|c|c|c|c|c|c|c|c|c|c|c|}
\hline \multirow{2}{*}{$\begin{array}{l}\text { Sp. } \\
\text { no. }\end{array}$} & \multirow{2}{*}{ Species } & \multicolumn{2}{|c|}{$\begin{array}{l}\text { Standard } \\
\text { length } \\
(\mathrm{cm}) \\
\mathrm{N}=10\end{array}$} & \multicolumn{2}{|c|}{$\begin{array}{c}\text { Total } \\
\text { length } \\
(\mathrm{cm}) \\
\mathrm{N}=10\end{array}$} & \multicolumn{2}{|c|}{ 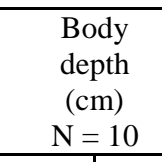 } & \multicolumn{2}{|c|}{ 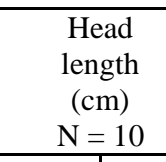 } & \multicolumn{2}{|c|}{$\begin{array}{c}\text { Weight } \\
\text { (g) } \\
\mathrm{N}=10\end{array}$} \\
\hline & & 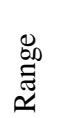 & $\begin{array}{l}\text { के } \\
+1 \\
\sum\end{array}$ & 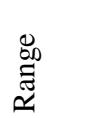 & $\begin{array}{l}0 \\
\omega \\
+1 \\
\sum\end{array}$ & $\begin{array}{l}\text { 吅 } \\
\text { ॠี }\end{array}$ & $\begin{array}{l}0 \\
\infty \\
+1 \\
\sum\end{array}$ & 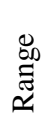 & $\begin{array}{l}\text { 命 } \\
\sum_{\Sigma}^{+1}\end{array}$ & $\begin{array}{l}\text { 焉 } \\
\sum_{\Sigma}^{+1}\end{array}$ & $\begin{array}{l}00 \\
\text { की } \\
\sum_{\Sigma}^{+1}\end{array}$ \\
\hline 1. & $\begin{array}{l}\text { Lutjanus } \\
\text { argentimaculatus }\end{array}$ & 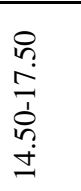 & \begin{tabular}{l} 
g. \\
0 \\
+1 \\
\multirow{1}{-1}{} \\
0 \\
-1
\end{tabular} & 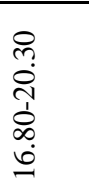 & 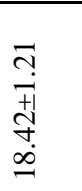 & 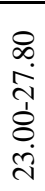 & $\begin{array}{l}8 \\
\stackrel{8}{.0} \\
\text { +1 } \\
\text { 㟔 } \\
\stackrel{+}{+}\end{array}$ & 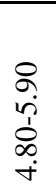 & $\begin{array}{l}\text { mे } \\
0 \\
\text { +1 } \\
\text { m. } \\
\text { iे }\end{array}$ & 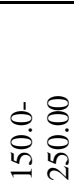 & 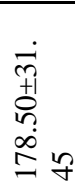 \\
\hline 2. & $\begin{array}{l}\text { Johnius } \\
\text { dussumieri }\end{array}$ & 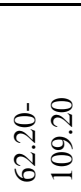 & 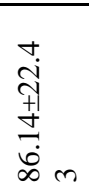 & 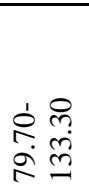 & $\begin{array}{l}\dot{0} \\
\text { 11 } \\
\dot{y} \\
\dot{\dot{\theta}} \\
\dot{\theta}\end{array}$ & 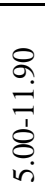 & 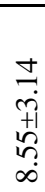 & 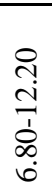 & 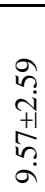 & 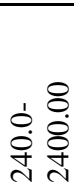 & 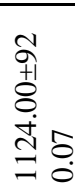 \\
\hline 3. & $\begin{array}{l}\text { Tachysurus } \\
\text { thalassinus }\end{array}$ & 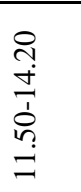 & 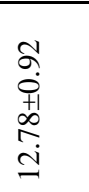 & 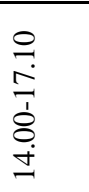 & 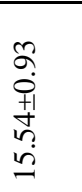 & 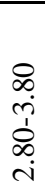 & 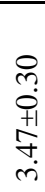 & 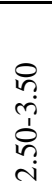 & 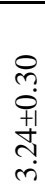 & 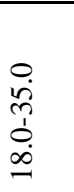 & 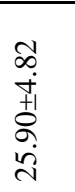 \\
\hline 4. & $\begin{array}{l}\text { Stolephorus } \\
\text { commersonnii }\end{array}$ & 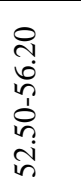 & 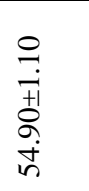 & 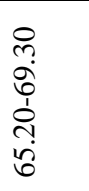 & $\begin{array}{l}\text { Nิ } \\
+1 \\
+1 \\
\hat{n} \\
\infty \\
0\end{array}$ & 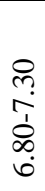 & \begin{tabular}{l} 
Nิ \\
0 \\
\multirow{1}{0}{} \\
$\stackrel{0}{n}$
\end{tabular} & 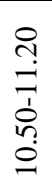 & $\begin{array}{l}\text { I } \\
\text { o } \\
\text { +1 } \\
\text {. } \\
ت\end{array}$ & 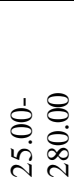 & 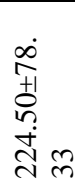 \\
\hline 5. & $\begin{array}{l}\text { Trichiurus } \\
\text { lepturus }\end{array}$ & 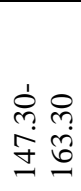 & $\begin{array}{l}0 \\
\stackrel{+}{+} \\
+1 \\
\stackrel{+}{+} \\
\text { N. } \\
\stackrel{\leftrightarrow}{\sim} \\
\rightarrow\end{array}$ & 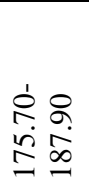 & 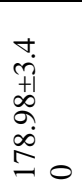 & 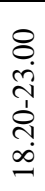 & 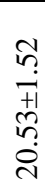 & $\begin{array}{l}\infty \\
\infty \\
0 \\
0 \\
\vdots \\
\infty \\
1 \\
0\end{array}$ & $\begin{array}{l}0 \\
0 \\
0 \\
+1 \\
0 \\
\oplus \\
\oplus\end{array}$ & 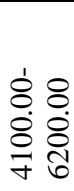 & 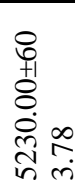 \\
\hline 6. & Lates calcarifer & 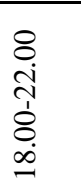 & 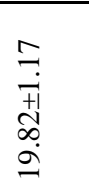 & 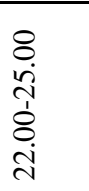 & $\begin{array}{l}\text { oे } \\
\ddot{+} \\
\text { +1 } \\
\text { m. } \\
\text { ָें }\end{array}$ & 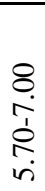 & 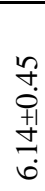 & 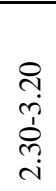 & $\begin{array}{l}\text { m. } \\
0 \\
+1 \\
0 \\
0 \\
\text { in }\end{array}$ & $\begin{array}{l}\text { oे : } \\
\text { : } \\
\text { : }\end{array}$ & 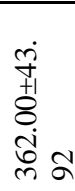 \\
\hline 7. & $\begin{array}{l}\text { Rastrelliger } \\
\text { kanagurta }\end{array}$ & 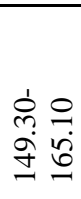 & $\begin{array}{l}0 \\
\dot{0} \\
+1 \\
0 \\
0 \\
\dot{0} \\
\stackrel{1}{-1} \\
-\end{array}$ & 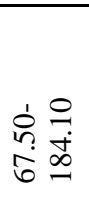 & 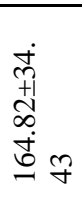 & $\begin{array}{l}\stackrel{P}{P} \\
\dot{+} \\
\dot{1} \\
\infty \\
\dot{0} \\
\dot{9}\end{array}$ & $\begin{array}{l}\mathcal{I} \\
\underset{+1}{+1} \\
\infty \\
\infty \\
\end{array}$ & 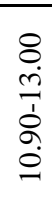 & 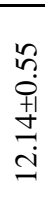 & $\begin{array}{l}8.8 \\
\stackrel{8}{8} \\
\stackrel{8}{8} \\
\text { 今 }\end{array}$ & 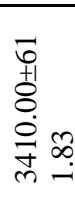 \\
\hline 8. & $\begin{array}{l}\text { Scomberomorus } \\
\text { commerson }\end{array}$ & $\begin{array}{l}8 \\
\infty \\
m \\
\dot{1} \\
0 \\
\dot{m} \\
\dot{m}\end{array}$ & 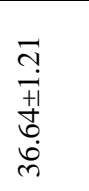 & $\begin{array}{l}8 \\
0 \\
\dot{0} \\
\dot{1} \\
o \\
-\dot{7} \\
\dot{\forall}\end{array}$ & 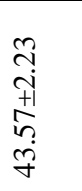 & 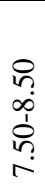 & $\begin{array}{l}\text { Nे } \\
0 \\
\text { †1 } \\
0 \\
\infty \\
\infty\end{array}$ & $\begin{array}{l}8 \\
\infty \\
0 \\
\vdots \\
0 \\
0 \\
0\end{array}$ & $\begin{array}{l}\frac{\sigma}{0} \\
\text { +1 } \\
\text { m. } \\
\text { के }\end{array}$ & 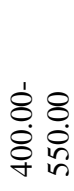 & 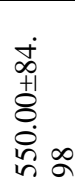 \\
\hline
\end{tabular}


Table 5 (continued): Morphometric characteristics of common marine fin fish and shell-fish species landing at Visakhapatnam Fishing Harbour.

\begin{tabular}{|c|c|c|c|c|c|c|c|c|c|c|c|}
\hline \multirow{2}{*}{$\begin{array}{l}\text { Sp. } \\
\text { no. }\end{array}$} & \multirow{2}{*}{ Species } & \multicolumn{2}{|c|}{$\begin{array}{c}\text { Standard } \\
\text { length } \\
(\mathrm{cm}) \\
\mathrm{N}=10 \\
\end{array}$} & \multicolumn{2}{|c|}{$\begin{array}{c}\text { Total } \\
\text { length } \\
(\mathrm{cm}) \\
\mathrm{N}=10\end{array}$} & \multicolumn{2}{|c|}{$\begin{array}{c}\begin{array}{c}\text { Body } \\
\text { depth } \\
(\mathrm{cm}) \\
\mathrm{N}=10\end{array}\end{array}$} & \multicolumn{2}{|c|}{$\begin{array}{c}\begin{array}{c}\text { Head } \\
\text { length } \\
(\mathrm{cm}) \\
\mathrm{N}=10\end{array} \\
\end{array}$} & \multicolumn{2}{|c|}{$\begin{array}{c}\text { Weight } \\
\text { (g) } \\
\mathrm{N}=10\end{array}$} \\
\hline & & $\begin{array}{l}\text { 品 } \\
\text { ॠ్ }\end{array}$ & $\begin{array}{l}0 \\
\infty \\
+1 \\
\sum\end{array}$ & 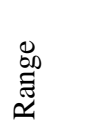 & $\begin{array}{l}0 \\
\infty \\
+1 \\
\sum\end{array}$ & $\begin{array}{l}\text { \&. } \\
\text { जี }\end{array}$ & $\begin{array}{l}0 \\
\infty \\
+1 \\
\sum\end{array}$ & $\begin{array}{l}\text { 品 } \\
\text { ๕్ }\end{array}$ & $\begin{array}{l}0 \\
\infty \\
\sum_{2}^{+1}\end{array}$ & $\begin{array}{l}\text { Dे } \\
\text { 芒 }\end{array}$ & $\begin{array}{l}0 \\
0 \\
\sum_{\Sigma}^{+1}\end{array}$ \\
\hline 9. & $\begin{array}{l}\text { Scomberomorus } \\
\text { guttatus }\end{array}$ & 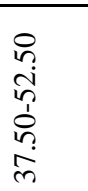 & $\begin{array}{l}\vec{m} \\
\dot{\omega} \\
+1 \\
0 \\
0 \\
\dot{y}\end{array}$ & 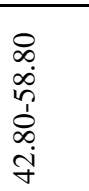 & $\begin{array}{l}\text { o } \\
\dot{\varphi} \\
+1 \\
0 \\
\dot{+} \\
\dot{q}\end{array}$ & \begin{tabular}{l}
8 \\
\multirow{1}{*}{} \\
तें \\
$\infty$
\end{tabular} & 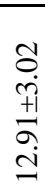 & $\begin{array}{l}\infty \\
\infty \\
10 \\
\overline{1} \\
8 \\
0 \\
0\end{array}$ & 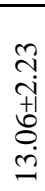 & 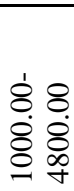 & 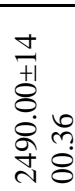 \\
\hline 10. & $\begin{array}{l}\text { Katsuwonus } \\
\text { pelamis }\end{array}$ & 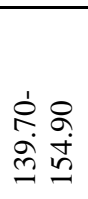 & 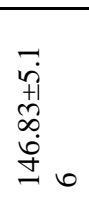 & 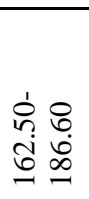 & $\begin{array}{l}\infty \\
0 \\
0 \\
+1 \\
0 \\
0 \\
\stackrel{0}{-1} \\
-\end{array}$ & 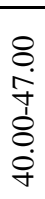 & 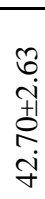 & 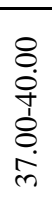 & 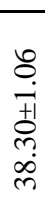 & 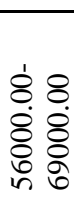 & 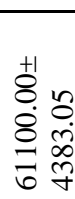 \\
\hline 11. & $\begin{array}{l}\text { Thunnus } \\
\text { albacares }\end{array}$ & 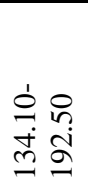 & 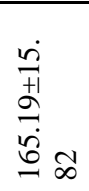 & 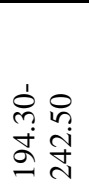 & 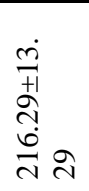 & 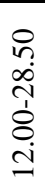 & 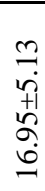 & 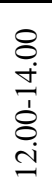 & 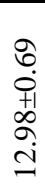 & 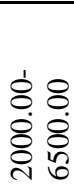 & 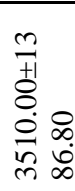 \\
\hline 12. & $\begin{array}{l}\text { Coryphaena } \\
\text { hippurus }\end{array}$ & 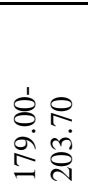 & 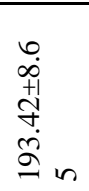 & 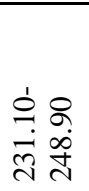 & 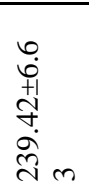 & 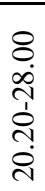 & 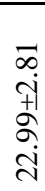 & $\begin{array}{l}8 \\
\dot{+} \\
\text { के } \\
\text { ठ். } \\
\dot{m}\end{array}$ & \begin{tabular}{l}
\multirow{3}{*}{} \\
+1 \\
$\hat{+1}$ \\
$\vec{m}$
\end{tabular} & 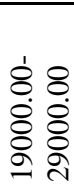 & 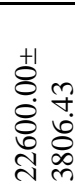 \\
\hline 13. & $\begin{array}{l}\text { Makaira } \\
\text { indica }\end{array}$ & 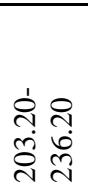 & 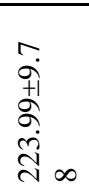 & 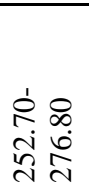 & 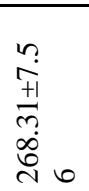 & 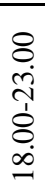 & 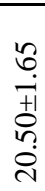 & $\begin{array}{l}8 \\
0 \\
\infty \\
0 \\
\vdots \\
\vdots \\
\infty \\
\infty\end{array}$ & $\begin{array}{l}? \\
0 \\
0 \\
+1 \\
+ \\
\infty \\
\infty \\
\infty\end{array}$ & 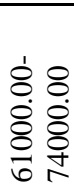 & 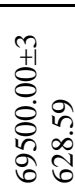 \\
\hline 14. & $\begin{array}{l}\text { Xiphias } \\
\text { gladius }\end{array}$ & 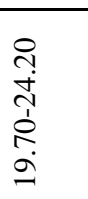 & $\begin{array}{l}8 \\
\stackrel{8}{1} \\
+1 \\
\stackrel{1}{N} \\
\stackrel{N}{i}\end{array}$ & 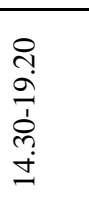 & 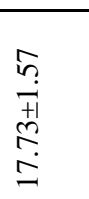 & 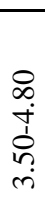 & 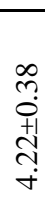 & $\begin{array}{l}\stackrel{0}{1} \\
\stackrel{1}{1} \\
\stackrel{1}{0} \\
0\end{array}$ & 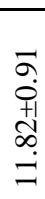 & 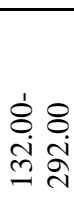 & 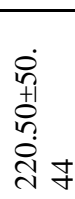 \\
\hline 15. & $\begin{array}{l}\text { Pampus } \\
\text { argenteus }\end{array}$ & $\begin{array}{l}8 \\
\dot{+} \\
\hat{m} \\
\dot{0} \\
\dot{2}\end{array}$ & $\begin{array}{l}8 \\
0 \\
+ \\
+1 \\
0 \\
0 \\
\dot{0} \\
\text { j. }\end{array}$ & 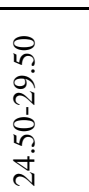 & 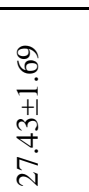 & $\begin{array}{l}R \\
0 \\
\vdots \\
\vdots \\
0\end{array}$ & 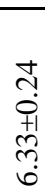 & 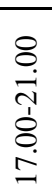 & 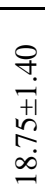 & 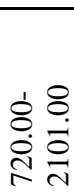 & 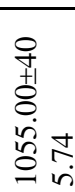 \\
\hline 16. & $\begin{array}{l}\text { Pampus } \\
\text { chinensis }\end{array}$ & 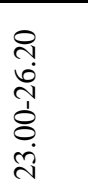 & 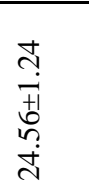 & 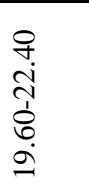 & 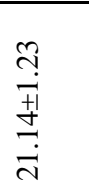 & $\begin{array}{l}8 \\
\dot{0} \\
\dot{1} \\
\dot{+} \\
\text { மं }\end{array}$ & 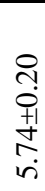 & 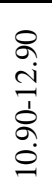 & 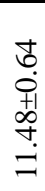 & 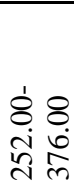 & $\begin{array}{l}\dot{0} \\
0 \\
+1 \\
\stackrel{1}{2} \\
\infty \\
\stackrel{N}{N} \\
\stackrel{N}{N}\end{array}$ \\
\hline
\end{tabular}


Table 5 (continued): Morphometric characteristics of common marine fin fish and shell-fish species landing at Visakhapatnam Fishing Harbour.

\begin{tabular}{|c|c|c|c|c|c|c|c|c|c|c|c|}
\hline \multirow{2}{*}{$\begin{array}{l}\text { Sp. } \\
\text { no. }\end{array}$} & \multirow{2}{*}{ Species } & \multicolumn{2}{|c|}{$\begin{array}{c}\text { Standard } \\
\text { length } \\
(\mathrm{cm}) \\
\mathrm{N}=10 \\
\end{array}$} & \multicolumn{2}{|c|}{$\begin{array}{c}\text { Total } \\
\text { length } \\
(\mathrm{cm}) \\
\mathrm{N}=10\end{array}$} & \multicolumn{2}{|c|}{ 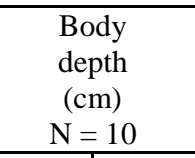 } & \multicolumn{2}{|c|}{$\begin{array}{c}\text { Head } \\
\text { length } \\
(\mathrm{cm}) \\
\mathrm{N}=10 \\
\end{array}$} & \multicolumn{2}{|c|}{$\begin{array}{c}\text { Weight } \\
\text { (g) } \\
\mathrm{N}=10\end{array}$} \\
\hline & & 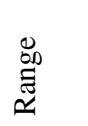 & $\begin{array}{l}0 \\
\infty \\
+1 \\
\sum\end{array}$ & 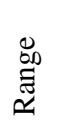 & $\begin{array}{l}0 \\
\omega \\
+1 \\
\sum\end{array}$ & 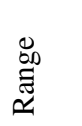 & $\begin{array}{l}0 \\
\infty \\
+1 \\
\sum\end{array}$ & 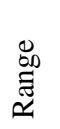 & $\begin{array}{l}0 \\
\text { in } \\
\sum_{\Sigma}^{+1}\end{array}$ & $\begin{array}{l}\text { जि } \\
\text { in } \\
\sum_{\Sigma}^{+1}\end{array}$ & $\begin{array}{l}0 \\
\infty \\
\sum_{\Sigma}^{+1}\end{array}$ \\
\hline 17. & $\begin{array}{l}\text { Parastromateus } \\
\text { niger }\end{array}$ & 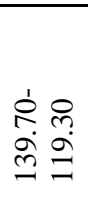 & 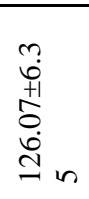 & 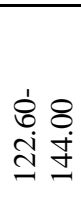 & $\begin{array}{l}\stackrel{\sigma}{0} \\
0 \\
+1 \\
0 \\
0 \\
\dot{\vec{m}} \\
\stackrel{-}{-1}\end{array}$ & 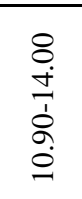 & 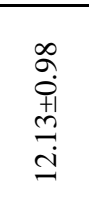 & $\begin{array}{l}8 \\
\infty \\
\sim \\
i \\
o \\
\dot{\sim}\end{array}$ & 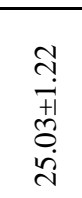 & 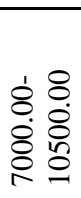 & 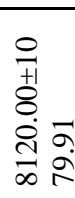 \\
\hline 18. & $\begin{array}{l}\text { Muraenesox } \\
\text { talabonoides }\end{array}$ & 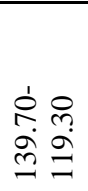 & 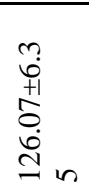 & 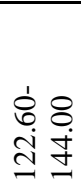 & 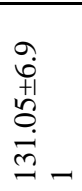 & 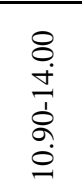 & $\begin{array}{l}\infty \\
0 \\
0 \\
+1 \\
m \\
\stackrel{+}{-1} \\
\underset{1}{-1}\end{array}$ & 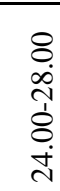 & 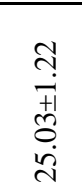 & $\begin{array}{l}18 \\
8 \\
80 \\
8 \\
8 \\
0\end{array}$ & 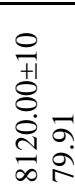 \\
\hline 19. & $\begin{array}{l}\text { Himantura } \\
\text { bleekeri }\end{array}$ & 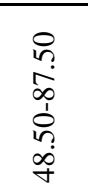 & $\begin{array}{l}9 \\
+ \\
+ \\
+1 \\
0 \\
0 \\
0 \\
0\end{array}$ & 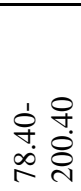 & 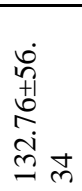 & 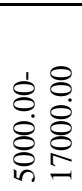 & $\begin{array}{l}m \\
+1 \\
0 \\
0 \\
\dot{0} \\
o \\
o \\
0 \\
0 \\
0\end{array}$ & 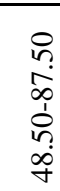 & $\begin{array}{l}9 \\
\dot{+} \\
+1 \\
0 \\
0 \\
0 \\
0\end{array}$ & 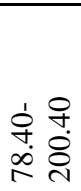 & 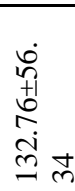 \\
\hline 20. & $\begin{array}{l}\text { Sepia } \\
\text { pharaonis }\end{array}$ & 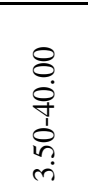 & $\begin{array}{l}\stackrel{L}{i} \\
\stackrel{+}{+1} \\
\stackrel{R}{2} \\
\stackrel{+}{\dot{J}} \sigma\end{array}$ & 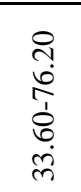 & 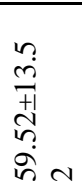 & $\begin{array}{l}1 \\
8 \\
0 \\
0 \\
0 \\
0\end{array}$ & $\begin{array}{l}0 \\
0 \\
+1 \\
+1 \\
0 \\
\infty \\
0 \\
0 \\
0\end{array}$ & 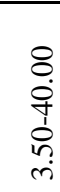 & 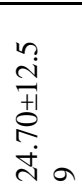 & $\begin{array}{l}\stackrel{1}{1} \\
0 \\
\hat{1} \\
\dot{1} \\
\dot{m} \\
m\end{array}$ & 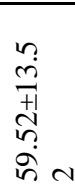 \\
\hline
\end{tabular}

Lutjanus argentimaculatus: The mean standard length was 19.71 while the mean total length was 23.60. The mean depth of body was 3.50 while the head length was 6.28 . The mean weight was 299.80 .

Johnius dussumieri: The mean standard length was 16.17 while the mean total length was 18.42. The mean depth of body was 24.85 while the head length was 5.31 . The mean weight was 178.50 .

Tachysurus thalassinus: The mean standard length was 86.14 while the mean total length was 106.04. The mean depth of body was 8.55 while the head length was 9.57 . The mean weight was 1,124.00.

Stolephorus commersonnii: The mean standard length was 12.78 while the mean total length was 15.54. The mean depth of body was 3.47 while the head length was 3.24 . The mean weight was 25.90.

Trichiurus lepturus: The mean standard length was 54.90 while the mean total length was 68.57 . The mean depth of body was 7.04 while the head length was 11.01 . The mean weight was 224.50 .

Lates calcarifer: The mean standard length was 153.45 while the mean total length was 178.98 . The mean depth of body was 20.53 while the head length was 16.36 . The mean weight was 5,230.00. 
Rastrelliger kanagurta: The mean standard length was 19.82 while the mean total length was 23.31. The mean depth of body was 6.14 while the head length was 2.60 . The mean weight was 362 .

Scomberomorus commerson: The mean standard length was 156.06 while the mean total length was 164.82. The mean depth of body was 12.83 while the head length was 12.14 . The mean weight was $3,410.00$.

Scomberomorus guttatus: The mean standard length was 36.64 while the mean total length was 43.57 . The mean depth of body was 8.03 while the head length was 9.31 . The mean weight was 550.00 .

Katsuwonus pelamis: The mean standard length was 44.07 while the mean total length was 49.01 . The mean depth of body was 12.91 while the head length was 13.06 . The mean weight was 2,490.00.

Thunnus albacares: The mean standard length was 146.83 while the mean total length was 176.59 . The mean depth of body was 42.70 while the head length was 38.30 . The mean weight was $61,100.00$.

Coryphaena hippurus: The mean standard length was 165.19 while the mean total length was 216.29. The mean depth of body was 16.95 while the head length was 12.98 . The mean weight was $3,510.00$.

Makaira indica: The mean standard length was 193.42 while the mean total length was 239.42. The mean depth of body was 22.99 while the head length was 31.07. The mean weight was 22,600.00.

Xiphias gladius: The mean standard length was 223.99 while the mean total length was 268.31. The mean depth of body was 20.50 while the head length was 88.40 . The mean weight was 69,500.00.

Pampus argenteus: The mean standard length was 22.75 while the mean total length was 17.73 . The mean depth of body was 4.22 while the head length was 11.82 . The mean weight was 220.50 .

Pampus chinensis: The mean standard length was 32.08 while the mean total length was 27.43. The mean depth of body was 6.33 while the head length was 18.75 . The mean weight was $1,055.00$.

Parastromateus niger: The mean standard length was 24.56 while the mean total length was 21.14. The mean depth of body was 5.74 while the head length was 11.48 . The mean weight was 298.70 .

Muraenesox talabonoides: The mean standard length was 126.07 while the mean total length was 131.05 . The mean depth of body was 12.13 while the head length was 25.03 . The mean weight was $8,120.00$.

Himantura bleekeri: The mean disc length was 68.97, the mean total length was 132.76 and the mean weight was 10,680.00.

Sepia pharaonis: The mean mantle length was 24.70 , the mean total length was 59.52 and the mean weight was 698.00 .

Physico-chemical characteristics of coastal water of Visakhapatnam: Coastal surface water collected at $500 \mathrm{~m}$ away from Visakhapatnam harbour was analyzed for certain physico-chemical characteristics showed that the mean $\mathrm{pH}$ value was 6.20 , the mean salinity value 24.51\%o, mean Dissolved Oxygen value $6.13 \mathrm{mg} / \mathrm{l}$, mean Biological Oxygen Demand $2.72 \mathrm{mg} / \mathrm{l}$ and mean temperature value $26.15^{\circ} \mathrm{C}$. The range and standard deviation values are included in table 6 . 
Table 6: Physico-chemical characteristics of coastal surface water collected 500 meters away from Visakhapatnam harbour.

\begin{tabular}{|c|l|c|c|}
\hline $\begin{array}{c}\text { Sp. } \\
\text { no. }\end{array}$ & \multicolumn{1}{|c|}{ Parameters } & $\begin{array}{c}\text { Range } \\
\mathrm{N}=10\end{array}$ & $\begin{array}{c}\text { Mean } \pm \text { SD } \\
\mathrm{N}=10\end{array}$ \\
\hline 1. & $\mathrm{pH}$ & $6.10-6.30$ & $6.20 \pm 0.07$ \\
\hline 2. & $\begin{array}{l}\text { Salinity } \\
(\%)\end{array}$ & $24.40-24.60$ & $24.51 \pm 0.06$ \\
\hline 3. & Dissolved oxygen $(\mathrm{mg} / \mathrm{l})$ & $6.00-6.30$ & $6.13 \pm 0.08$ \\
\hline 4. & $\begin{array}{l}\text { BOD } \\
(\mathrm{mg} / \mathrm{l})\end{array}$ & $2.60-2.80$ & $2.72 \pm 0.06$ \\
\hline 5. & $\begin{array}{l}\text { Temperature } \\
\left({ }^{\circ} \mathrm{C}\right)\end{array}$ & $26.00-26.50$ & $26.15 \pm 0.14$ \\
\hline
\end{tabular}

Morphological characteristics of Pampus argenteus: Body colour is silvery white on side and blue to grey on the back. Fins are yellowish with dark edges. Body is very deep and compressed but fairly thick. Caudal peduncle is short, deep, and compressed. Mouth is small, sub-terminal and curved downwards with immobile maxilla which are covered with skin and united with cheek. Teeth are minute, uni-seriate, and flattened with a large central cusp and a toothless palate. Gill membranes are broadly attached to isthmus, while gill rakers are twothree to eight-ten on the first arch. Dorsal fin rays are 38-43 while anal fin rays are 34-43. Pectoral fins are long while pelvic fins are absent. Dorsal and anal fins are approximately equal in length; they originate ahead of mid body but are behind pectoral fin base and preceded by fife-ten low blade like spines which are pointed on both ends. Caudal fin is stiff and forked, and the lower caudal fin is like a lobe. Anterior rays, anal fins, median fins, ventral lobe of caudal fins are elongated and curved in a sickle-shaped manner.

Morphological characteristics of Pampus chinensis: Body colour is grey to brown on the back, silver white on the sides and small dots on the body. Fins are yellowish to dusky. Body is very deep. Caudal peduncle is short, deep and compressed without keels. Mouth is small, curved downwards with immobile maxilla covered with skin and united with cheek. Jaw teeth are minute, uni-seriate and flattened with a large central cusp and two smaller cusps and a toothless palate. Gill membranes are broadly attached with isthmus while gill opening is a straight vertical slit covered with a flap of skin. Dorsal fin rays 43 to 50 while anal fin rays are 39-42. Dorsal and anal fins are sub-equal in length, which originate at the level of or behind pectoral fin base and without spines ahead of fins. Pectoral fins are broad while pelvic finds are absent. Caudal fin is broad and slightly forked. Median fin rays create almost a vertical margin at posterior border of the fins.

Morphological characteristics of Parastromateus niger: Body colour is uniformly silvery to bluish brown. Fins are with dark edges. Body is deep and compressed. Dorsal and ventral sides of the are body are equally convex. Mouth is terminal with upper jaw unrestricted dorsally and ends below slightly before anterior margin of eye. Both jaws possess a single row of small conical teeth. Gill opening is unrestricted both laterally and ventrally. Dorsal fin with four to five short spines followed by one spine plus 41 and 44 soft rays. Anal fin with two embedded spines followed by one spine plus 35 to 39 soft rays. Profile of second dorsal and anal fins is identical with elevated and broadly rounded anterior lobes. Pelvic fins are absent in individuals larger than $10 \mathrm{~cm}$ fork and falcate. Lateral line is weakly arched anteriorly and accompanied by a junction of straight and curved parts below the posterior third of dorsal fin; straight part of lateral line has eight to 19 weak scutes which form a keel on caudal peduncle. 
A comparison of the morphological features of the three Pomfret fish species showed that $P$. chinensis have higher values in the measured features indicating that this species has more muscle and more weight. The next fish species in terms of more weight is $P$. niger and finally $P$. argenteus.

Table 7: Morphometric features of three selected Pomfret fish species.

\begin{tabular}{|c|c|c|c|c|c|c|c|}
\hline \multirow{2}{*}{$\begin{array}{l}\text { S. } \\
\text { no. }\end{array}$} & \multirow{2}{*}{ Parameters } & \multicolumn{2}{|c|}{$\begin{array}{c}\text { Pampus argenteus } \\
\mathrm{N}=10\end{array}$} & \multicolumn{2}{|c|}{$\begin{array}{l}\text { Pampus chinensis } \\
\mathrm{N}=10\end{array}$} & \multicolumn{2}{|c|}{$\begin{array}{l}\text { Parastromateus niger } \\
\mathrm{N}=10\end{array}$} \\
\hline & & $\mathrm{M} \pm \mathrm{SD}$ & Range & $\mathrm{M} \pm \mathrm{SD}$ & Range & $\mathrm{M} \pm \mathrm{SD}$ & Range \\
\hline 1. & $\begin{array}{l}\text { Total length } \\
\text { (cm) }\end{array}$ & $\begin{array}{l}19.70- \\
24.20\end{array}$ & $\begin{array}{l}22.75 \pm \\
1.60\end{array}$ & $\begin{array}{l}29.00- \\
34.00\end{array}$ & $\begin{array}{l}32.08 \pm \\
1.69\end{array}$ & $\begin{array}{l}23.00- \\
26.20\end{array}$ & $\begin{array}{l}24.56 \pm \\
1.24\end{array}$ \\
\hline 2. & $\begin{array}{l}\text { Standard } \\
\text { length }(\mathrm{cm})\end{array}$ & $\begin{array}{l}14.30- \\
19.20\end{array}$ & $\begin{array}{l}17.73 \pm \\
1.57\end{array}$ & $\begin{array}{l}24.50- \\
29.50\end{array}$ & $\begin{array}{l}27.43 \pm \\
1.69\end{array}$ & $\begin{array}{l}19.60- \\
22.40\end{array}$ & $\begin{array}{l}21.14 \pm \\
1.23\end{array}$ \\
\hline 3. & $\begin{array}{l}\text { Head length } \\
(\mathrm{cm})\end{array}$ & $\begin{array}{l}3.50- \\
4.80\end{array}$ & $\begin{array}{l}4.22 \pm \\
0.38\end{array}$ & $\begin{array}{l}6.00- \\
6.70\end{array}$ & $\begin{array}{l}6.33 \pm \\
0.24\end{array}$ & $\begin{array}{l}5.40- \\
6.00\end{array}$ & $\begin{array}{l}5.74 \pm \\
0.20\end{array}$ \\
\hline 4. & $\begin{array}{l}\text { Body depth } \\
\text { (cm) }\end{array}$ & $\begin{array}{l}10.20- \\
13.00\end{array}$ & $\begin{array}{l}11.82 \pm \\
0.91\end{array}$ & $\begin{array}{l}17.00- \\
21.00\end{array}$ & $\begin{array}{l}18.75 \pm \\
1.40\end{array}$ & $\begin{array}{l}10.90- \\
12.90\end{array}$ & $\begin{array}{l}11.48 \pm \\
0.64\end{array}$ \\
\hline 5. & Weight & $\begin{array}{l}132.00- \\
292.00\end{array}$ & $\begin{array}{l}220.50 \pm \\
50.44\end{array}$ & $\begin{array}{l}720.00- \\
2101.00\end{array}$ & $\begin{array}{l}1055.00 \pm \\
405.74\end{array}$ & $\begin{array}{l}252.00- \\
376.00\end{array}$ & $\begin{array}{l}298.70 \pm \\
50.72\end{array}$ \\
\hline
\end{tabular}

Protein content in the muscle tissue of Pampus argenteus, $P$. chinensis and Parastromateus niger: The total protein content varied in each species. The mean total protein content was $18.28 \%$ in $P$. argenteus, $16.24 \%$ in $P$. chinensis and $19.58 \%$ in $P$. niger (Tab. 8). The range and standard deviation values are presented in table 8.

Table 8: Protein content in the muscle portion of three selected Pomfret fish species.

\begin{tabular}{|c|c|c|}
\hline \multirow{2}{*}{ Species } & \multicolumn{2}{|c|}{$\begin{array}{r}\text { Total protein }(\% \mathrm{w} / \mathrm{w}) \\
(\mathrm{N}=10)\end{array}$} \\
\cline { 2 - 3 } & Range & $18.28 \pm 1.51$ \\
\hline \multirow{2}{*}{ Pampus argenteus } & $15.44-20.42$ & $16.24 \pm 0.96$ \\
\hline Pampus chinensis & $14.61-17.88$ & $19.58 \pm 1.43$ \\
\hline Parastromateus niger & $17.61-21.08$ & \\
\hline Analysis method: Indian standard method (IS 7219-1973) \\
\hline
\end{tabular}


Concentrations of heavy metals in the muscle tissue and gill portions of Pampus argenteus, $\boldsymbol{P}$. chinensis and Parastromateus niger: The concentrations of heavy metals, arsenic, cadmium, mercury, and lead in muscle and gill portions of the stated fish species were detected using Inductively Coupled Plasma Mass Spectrometry (ICP MS). LOD value is 0.03 for arsenic and mercury, 0.06 for cadmium and 0.018 for lead. LOQ value is 0.10 for arsenic, cadmium, and mercury, and 0.06 for lead. In the muscle portion of all the three fish species, all four heavy metals were present. In $P$. argenteus, the mean concentration of arsenic was 0.81 $\mathrm{mg} / \mathrm{kg}$, of cadmium and mercury, each $0.5 \mathrm{mg} / \mathrm{kg}$ and of lead $0.08 \mathrm{mg} / \mathrm{kg}$. In $P$. chinensis, the mean concentration of arsenic was $2.80 \mathrm{mg} / \mathrm{kg}$, of cadmium and mercury, each $0.06 \mathrm{mg} / \mathrm{kg}$ and of lead $0.18 \mathrm{mg} / \mathrm{kg}$. In $P$. niger, the mean concentration of arsenic was $2.78 \mathrm{mg} / \mathrm{kg}$, of cadmium $0.06 \mathrm{mg} / \mathrm{kg}$, of mercury $0.05 \mathrm{mg} / \mathrm{kg}$, and of lead $0.08 \mathrm{mg} / \mathrm{kg}$. In the gill portion of all the three fish species, all four heavy metals were present. In $P$. argenteus, the mean concentration of arsenic was $0.72 \mathrm{mg} / \mathrm{kg}$, of cadmium $0.05 \mathrm{mg} / \mathrm{kg}$, of mercury $0.06 \mathrm{mg} / \mathrm{kg}$, and of lead $0.08 \mathrm{mg} / \mathrm{kg}$. In $P$. chinensis, the mean concentration of arsenic was $0.82 \mathrm{mg} / \mathrm{kg}$, of cadmium and mercury, each $0.5 \mathrm{mg} / \mathrm{kg}$ and of lead $0.06 \mathrm{mg} / \mathrm{kg}$. In $P$. niger, the mean concentration of arsenic was $1.33 \mathrm{mg} / \mathrm{kg}$, of cadmium and mercury, each $0.05 \mathrm{mg} / \mathrm{kg}$, and of lead $0.32 \mathrm{mg} / \mathrm{kg}$ (Tabs. 9-12).

Table 9: Recovery of arsenic, cadmium, mercury, and lead in the muscle portion of three selected Pomfret fish species.

\begin{tabular}{|c|c|c|c|c|c|c|c|c|c|}
\hline \multirow{2}{*}{ 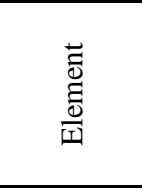 } & \multirow{2}{*}{ 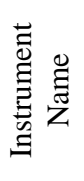 } & \multirow[b]{2}{*}{ Oి } & \multirow{2}{*}{$\underset{\nearrow}{~}$} & \multirow{2}{*}{ 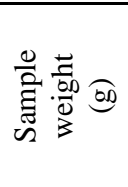 } & \multirow{2}{*}{ 产 } & \multicolumn{3}{|c|}{$\begin{array}{c}\text { Content of metal recovered (Mean } \\
\text { Conc.) in Muscle (mg/kg) }\end{array}$} & \multirow{2}{*}{$\begin{array}{c}\text { Recovery } \\
\% \\
\text { (has to be } \\
\text { in the } \\
\text { limits) }\end{array}$} \\
\hline & & & & & & $\begin{array}{l}\text { Pampus } \\
\text { argenteus }\end{array}$ & $\begin{array}{l}\text { Pampus } \\
\text { chinensis }\end{array}$ & $\begin{array}{c}\text { Parastro- } \\
\text { mateus } \\
\text { niger }\end{array}$ & \\
\hline Arsenic & \multirow{4}{*}{ 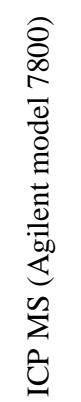 } & 0.03 & 0.10 & 0.5 & 0.5 & 0.81 & 2.80 & 2.78 & \multirow{4}{*}{$70-120 \%$} \\
\hline Cadmium & & 0.06 & 0.10 & 0.5 & 0.5 & 0.05 & 0.06 & 0.06 & \\
\hline Mercury & & 0.03 & 0.10 & 0.5 & 0.5 & 0.05 & 0.06 & 0.05 & \\
\hline Lead & & 0.018 & 0.06 & 0.5 & 0.5 & 0.08 & 0.18 & 0.08 & \\
\hline
\end{tabular}


Table 10: Recovery of arsenic, cadmium, mercury, and lead in the gill portion of three selected Pomfret fish species.

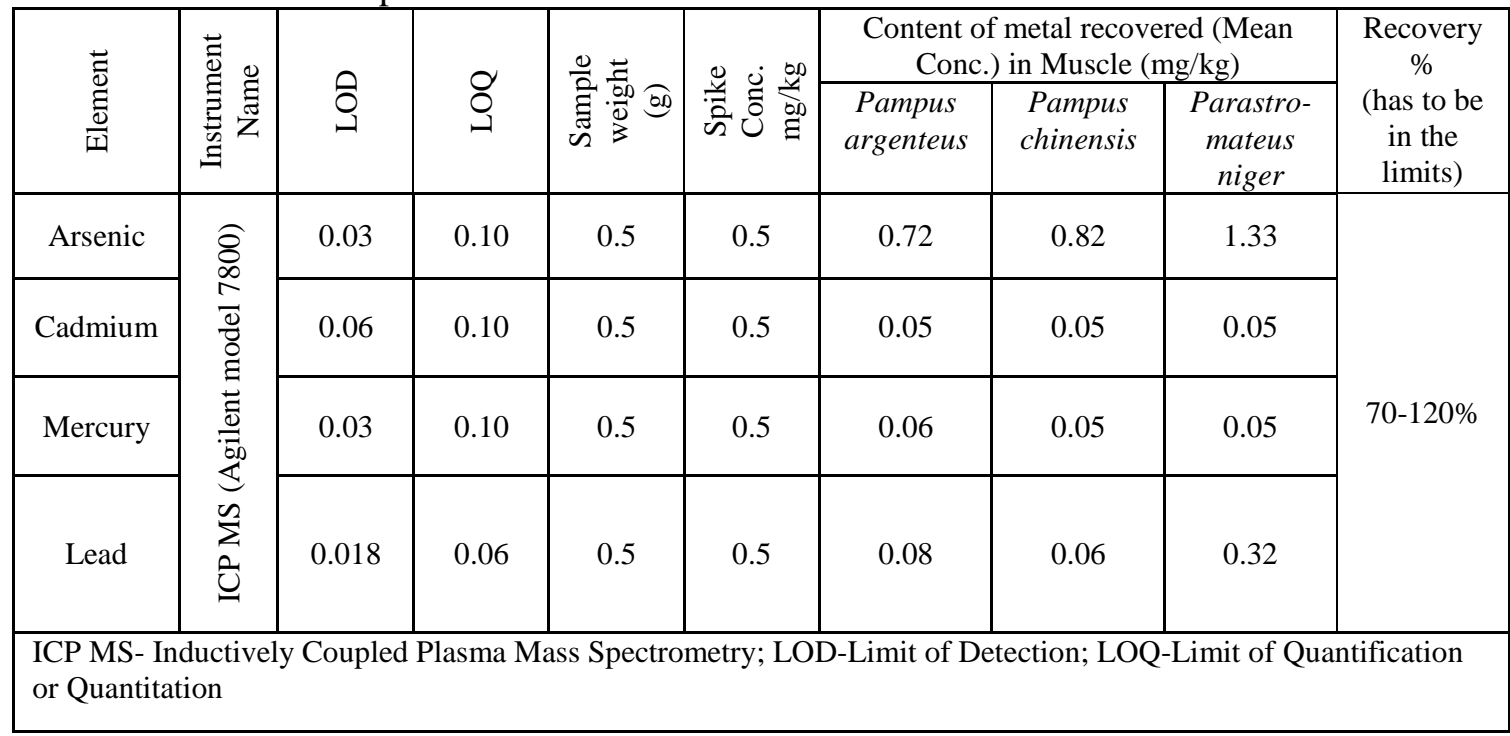

Table 11: Heavy metals concentrations in the muscle portion of three selected Pomfret fish species.

\begin{tabular}{|l|l|c|c|c|c|c|c|c|c|}
\hline \multirow{3}{*}{$\begin{array}{c}\text { S. } \\
\text { no. }\end{array}$} & \multirow{2}{*}{$\begin{array}{c}\text { Scientific } \\
\text { name }\end{array}$} & \multicolumn{2}{|c|}{$\begin{array}{c}\text { Arsenic } \\
(\mathrm{mg} / \mathrm{kg})\end{array}$} & \multicolumn{2}{c|}{$\begin{array}{c}\text { Cadmium } \\
(\mathrm{mg} / \mathrm{kg})\end{array}$} & \multicolumn{2}{c|}{$\begin{array}{c}\text { Mercury } \\
(\mathrm{mg} / \mathrm{kg})\end{array}$} & \multicolumn{2}{c|}{$\begin{array}{c}\text { Lead } \\
(\mathrm{mg} / \mathrm{kg})\end{array}$} \\
\cline { 3 - 10 } & & Range & $\mathrm{M} \pm \mathrm{SD}$ & Range & $\mathrm{M} \pm \mathrm{SD}$ & Range & $\mathrm{M} \pm \mathrm{SD}$ & Range & $\mathrm{M} \pm \mathrm{SD}$ \\
\hline 1. & Pampus & $0.58-$ & $0.81 \pm$ & $0.00-$ & $0.05 \pm$ & $0.04-$ & $0.05 \pm$ & $0.04-$ & $0.08 \pm$ \\
& argenteus & 1.14 & 0.18 & 0.05 & 0.00 & 0.09 & 0.01 & 0.11 & 0.01 \\
\hline 2. & Pampus & $1.87-$ & $2.80 \pm$ & $0.05-$ & $0.06 \pm$ & $0.05-$ & $0.06 \pm$ & $0.07-$ & $0.18 \pm$ \\
& chinensis & 5.14 & 1.24 & 0.11 & 0.02 & 0.12 & 0.02 & 0.5 & 0.12 \\
\hline 3. & Parastromateus & $1.85-$ & $2.78 \pm$ & $0.05-$ & $0.06 \pm$ & $0.05-$ & $0.05 \pm$ & $0.05-$ & $0.08 \pm$ \\
& niger & 3.82 & 0.65 & 0.08 & 0.01 & 0.05 & 0.00 & 0.12 & 0.03 \\
\hline
\end{tabular}

Table 12: Heavy metals concentrations in the gill portion of three selected Pomfret fish species.

\begin{tabular}{|l|l|c|c|c|c|c|c|c|c|}
\hline \multirow{3}{*}{$\begin{array}{c}\text { S. } \\
\text { no. }\end{array}$} & \multirow{2}{*}{$\begin{array}{c}\text { Scientific } \\
\text { name }\end{array}$} & \multicolumn{2}{|c|}{$\begin{array}{c}\text { Arsenic } \\
(\mathrm{mg} / \mathrm{kg})\end{array}$} & \multicolumn{2}{c|}{$\begin{array}{c}\text { Cadmium } \\
(\mathrm{mg} / \mathrm{kg})\end{array}$} & \multicolumn{2}{c|}{$\begin{array}{c}\text { Mercury } \\
(\mathrm{mg} / \mathrm{kg})\end{array}$} & \multicolumn{2}{c|}{$\begin{array}{c}\text { Lead } \\
(\mathrm{mg} / \mathrm{kg})\end{array}$} \\
\cline { 3 - 10 } & & Range & $\mathrm{M} \pm \mathrm{SD}$ & Range & $\mathrm{M} \pm \mathrm{SD}$ & Range & $\mathrm{M} \pm \mathrm{SD}$ & Range & $\mathrm{M} \pm \mathrm{SD}$ \\
\hline 1. & $\begin{array}{l}\text { Pampus } \\
\text { argenteus }\end{array}$ & $0.54-$ & $0.72 \pm$ & $0.05-$ & $0.05 \pm$ & $0.05-$ & $0.06 \pm$ & $0.07-$ & $0.08 \pm$ \\
& 0.91 & 0.12 & 0.05 & 0.00 & 0.10 & 0.02 & 0.12 & 0.02 \\
\hline 2. & Pampus & $0.50-$ & $0.82 \pm$ & $0.02-$ & $0.05 \pm$ & $0.05-$ & $0.06 \pm$ & $0.06-$ & $0.15 \pm$ \\
& chinensis & 1.42 & 0.33 & 0.09 & 0.02 & 0.13 & 0.03 & 0.24 & 0.06 \\
\hline 3. & Parastromateus & $0.79-$ & $1.33 \pm$ & $0.05-$ & $0.05 \pm$ & $0.05-$ & $0.05 \pm$ & $0.05-$ & $0.32 \pm$ \\
& niger & 2.13 & 0.46 & 0.07 & 0.01 & 0.05 & 0.00 & 1.44 & 0.53 \\
\hline
\end{tabular}


Comparison of heavy metal concentrations in muscle/gill portions of Pomfret fish species with the permissible standards set out by different organizations: Heavy metal concentrations detected in muscle and gill portions of all the three Pomfret fish species were compared with the recommended limits permitted by European Union Regulations (EU) 2006, Food and Agriculture Organization (FAO)/World Health Organization (WHO) 2011, Ministry of Agriculture, Fisheries and Food (MAFF) and Food Safety and Standards Authority of India (FSSAI). Standards for arsenic are not available in the regulations of EU (2006), FAO/WHO (2011) and MAFF. According to FSSAI (2011), the concentration of arsenic in muscle and gill portion separately or put together in all the three fish species was highly negligible and far below the recommended limit. For cadmium, according to EU (2006) and FAO (2003) regulations, the recommended limit is $0.05 \mathrm{mg} / \mathrm{kg}$; according to MAFF, the recommended limit is $0.2 \mathrm{mg} / \mathrm{kg}$; and according to FSSAI (2011), the recommended limit is $0.3 \mathrm{mg} / \mathrm{kg}$. The detected concentration of cadmium in the muscle and gill portion of $P$. argenteus, and in the gill portion of $P$. chinensis and $P$. niger was at the recommended limit and in the muscle portion of $P$. chinensis and $P$. niger slightly exceeded the recommended limit set by EU (2006) and FAO (2003) regulations. But the detected concentration of cadmium in the muscle and gills of all the three fish species exceeded the recommended limit set by MAFF and FSSAI (2011). The detected concentration of mercury in the muscle tissue of $P$. argenteus and in the muscles and gills of $P$. niger was within the recommended limit according to EU (2006), FAO/WHO (2011), FAO (2003), MAFF and FSSAI (2011). But the detected concentration of mercury in the gills of $P$. argenteus, muscle and gill portions of $P$. chinensis slightly exceeded the permissible limit set out by all these regulating organizations. The detected concentration of lead in the muscle and gill portion of $P$. argenteus, $P$. chinensis, and in the muscle portion of $P$. niger were lower than the allowed limit indicated by all these regulating organizations. But the detected concentration of lead in the gill portion of $P$. niger slightly exceeded the allowed limit indicated by EU (2006), FAO/WHO (2011) and FSSAI (2011) and prominently exceeded the allowed limit set out by FAO (2003) and MAFF (Tab. 13).

Table 13: Comparison of heavy metal concentrations analyzed in muscle and gill portions of three selected Pomfret fish species with the permissible standards set out by different organizations.

\begin{tabular}{|c|c|c|c|c|c|c|c|c|c|c|c|c|}
\hline \multirow{2}{*}{$\begin{array}{l}\dot{0} \\
\dot{0} \\
\dot{\omega}\end{array}$} & \multirow{2}{*}{ 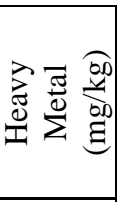 } & \multicolumn{2}{|c|}{$\begin{array}{c}\text { Pampus } \\
\text { argenteus } \\
(\mathrm{N}=10)\end{array}$} & \multicolumn{2}{|c|}{$\begin{array}{l}\text { Pampus } \\
\text { chinensis } \\
(\mathrm{N}=10)\end{array}$} & \multicolumn{2}{|c|}{$\begin{array}{c}\text { Parastroma- } \\
\text { teus niger } \\
(\mathrm{N}=10)\end{array}$} & \multirow{2}{*}{$\begin{array}{c}\text { E.U } \\
2006 \\
\mathrm{mg} / \\
\mathrm{kg}\end{array}$} & \multirow{2}{*}{$\begin{array}{c}\mathrm{FAO} / \\
\mathrm{WHO} \\
2011 \\
\mathrm{mg} / \\
\mathrm{kg}\end{array}$} & \multirow{2}{*}{$\begin{array}{c}\text { FAO } \\
2003 \\
\mathrm{mg} / \\
\mathrm{kg}\end{array}$} & \multirow[t]{2}{*}{$\begin{array}{c}\text { MAFF } \\
\mathrm{mg} / \\
\mathrm{kg}\end{array}$} & \multirow{2}{*}{$\begin{array}{c}\text { FSSAI } \\
2011 \\
\mathrm{mg} / \\
\mathrm{kg}\end{array}$} \\
\hline & & Muscle & Gills & Muscle & Gills & Muscle & Gills & & & & & \\
\hline 1 & $\begin{array}{l}\text { Ar- } \\
\text { senic }\end{array}$ & $\begin{array}{c}0.81 \\
\pm \\
0.18\end{array}$ & $\begin{array}{c}0.72 \\
\pm \\
0.12\end{array}$ & $\begin{array}{c}2.80 \\
\pm \\
1.24\end{array}$ & $\begin{array}{c}0.82 \\
\pm \\
0.33\end{array}$ & $\begin{array}{c}2.78 \\
\pm \\
0.65\end{array}$ & $\begin{array}{c}1.33 \\
\pm \\
0.46\end{array}$ & - & - & - & - & 76 \\
\hline 2 & $\begin{array}{l}\text { Cad- } \\
\text { mium }\end{array}$ & $\begin{array}{c}0.05 \\
\pm \\
0.00\end{array}$ & $\begin{array}{c}0.05 \\
\pm \\
0.00\end{array}$ & $\begin{array}{c}0.06 \\
\pm \\
0.02\end{array}$ & $\begin{array}{c}0.05 \\
\pm \\
0.02\end{array}$ & $\begin{array}{c}0.06 \\
\pm \\
0.01\end{array}$ & $\begin{array}{c}0.05 \\
\pm \\
0.01\end{array}$ & 0.05 & - & 0.05 & 0.2 & 0.3 \\
\hline 3 & $\begin{array}{l}\text { Mer- } \\
\text { cury }\end{array}$ & $\begin{array}{c}0.05 \\
\pm \\
0.01 \\
\end{array}$ & $\begin{array}{c}0.06 \\
\pm \\
0.02\end{array}$ & $\begin{array}{c}0.06 \\
\pm \\
0.02 \\
\end{array}$ & $\begin{array}{c}0.06 \\
\pm \\
0.03\end{array}$ & $\begin{array}{c}0.05 \\
\pm \\
0.00\end{array}$ & $\begin{array}{c}0.05 \\
\pm \\
0.00\end{array}$ & 0.5 & 0.5 & 0.5 & 0.5 & 0.5 \\
\hline 4 & Lead & $\begin{array}{c}0.08 \\
\pm \\
0.01 \\
\end{array}$ & $\begin{array}{c}0.08 \\
\pm \\
0.02\end{array}$ & $\begin{array}{c}0.18 \\
\pm \\
0.12 \\
\end{array}$ & $\begin{array}{c}0.15 \\
\pm \\
0.06\end{array}$ & $\begin{array}{c}0.08 \\
\pm \\
0.03 \\
\end{array}$ & $\begin{array}{c}0.32 \\
\pm \\
0.53 \\
\end{array}$ & 0.3 & 0.3 & 0.2 & 2.0 & 0.3 \\
\hline
\end{tabular}

E.U: European Union regulations; FAO: Food and Agriculture Organization; WHO: World Health Organization; MAFF: Ministry of Agriculture, Fisheries and Food; FSSAI- Food Safety and Standards Authority of India. 


\section{DISCUSSION}

Inventory of marine fishery resources of coastline of Andhra Pradesh: The State of Andhra Pradesh has a long coastline of $974 \mathrm{~km}$ and a continental shelf area of $33,227 \mathrm{~km}^{2}$ which is spread over nine coastal districts. This coastline area is the home for a diversity of marine fishery resources comprising of several groups of fishes, crustaceans, molluscs, and other marine organisms. This coastline of Andhra Pradesh is known for a thriving fisheries sector which provides nutritional food to the people and exports surplus seafood to other countries. The fish catches fluctuate greatly from year to year depending on the spawning potential, consumption levels, and pollution factors (Syda Rao et al., 2008). Despite the great potential for fishery resources, there is no consolidated list of fin fishes, crustaceans, and molluscs for the coastline of Andhra Pradesh in order to evaluate the potential of different stations across the coastline to provide seafood for locals, export seafood to other countries and provide livelihood opportunities for fishing community. In this study, an inventory of fishery resources of coastline of Andhra Pradesh prepared based on field study indicated that a total of 212 species consisting of finfishes, crustaceans, and molluscs. Among fin fishes, 26 species belong to Elasmobranch group, 146 species to Teleosts group. The crustaceans comprised of 30 species while molluscs comprised of 10 species. Fin fishes and crustaceans have been found to be the major contributors to fishery sector while the contribution of molluscs to the fishery sector is negligible. Sharks are more speciose than skates and rays among Elasmobranchs while shads, sardines, anchovies, carangids, and croakers are more speciose among Teleosts. Penaeids are more speciose among crustaceans. Among molluscs, bivalves, and cephalods comprised of a few species of which the cephalod, Sepia pharaonis is the only species that has commercial and export value.

Common marine fishery resources of Visakhapatnam coast: Muddula Krishna et al. (2016) reported 28 fish species from the Visakhapatnam coastal waters, of which eight species belong to threatened categories of IUCN red list status. In this study, it is found that twenty edible marine species land at the Fishing Harbour and local markets of Visakhapatnam. They are Himantura bleekeri, Lutjanus argentimaculatus, Johnius dussumieri, Tachysurus thalassinus, Stolephorus commersonii, Trichiurus lepturus, Lates calcarifer, Rastrelliger kanagurta, Scomberomorus commerson, S. guttatus, Katsuwonus pelamis, Thunnus albacares, Coryphaena hippurus, Makaira indica, Xiphias gladius, Pampus argenteus, P. chinensis, Parastromateus niger, Muraenesox talabonoides, and Sepia pharaonis. According to IUCN red list, S. commerson and T. albacares are classified under Near Threatened category; S. guttatus, M. indica, and S. pharaonis under Data Deficient category; H. bleekeri, T. thalassinus, R. kanagurta, $P$. argenteus, $P$. chinensis, and $M$. talabonoides under Not Evaluated category; and all other species under Least Concern category. In the last category, $X$. gladius and $P$. niger have been stated as populations are decreasing. Among these twenty species, the species under Near Threatened category although do not qualify for the Threatened Status need to be evaluated from time to time to revise their status. The species under Data Deficient category need sufficient fact-based information for the evaluation of their current status. The species under Not Evaluated category require immediate evaluation of their present status as IUCN has not assessed their status so far. The species under Least Concern category do not need any evaluation for their conservation, however, regular evaluation of these species is a necessity as their populations may dwindle gradually as recorded in case of Xiphias gladius and Parastromateus niger by IUCN. The present study shows that 
the populations of these species and accordingly their status are consistently subjected to degradation and fragmentation of their habitats due to unorganized and organized human activities involving urbanization, industrialization and tourism. Further, over-exploitation and over-consumption locally and also seafood exports through Visakhapatnam Sea Food Export Trade Center (VSFETC) contribute to the rapid decline of populations of these species. Therefore, there is an urgent need to evaluate the present status of the edible marine species of Visakhapatnam coastal waters in order to take proper measures for their conservation, management and utilization as food and revenue sources.

Gears used for catching marine fishery resources from Visakhapatnam coast: Sreekrishna (2002) provided the details of different crafts operating to harvest marine fishery resources along the coastline of Andhra Pradesh. Syda Rao et al. (2008) reported that a variety of gears are employed to harvest the coastal and offshore fishery resources of Andhra Pradesh state. These authors noted that the gears in use range from simple cast nets to large seines and trawls. The line fishing is very active for coastal, offshore and oceanic fishes in Andhra Pradesh. These gears are operated from small traditional non-mechanized crafts, motorized crafts and medium to large mechanized crafts depending on the area of operation, targeted fishes and the fish price as well. Immanuel and Syda Rao (2012) reported that long line with 500-600 hooks and hand line with six to ten hooks are important gears used by fishermen involved in harvesting fish from coastal waters of Visakhapatnam. In the present study, the fishermen of the coastline of Visakhapatnam use different gears to harvest marine fishery resources. They belong to Surrounding nets, Seine nets, Trawls, Lift nets, Gillnets and Entangling nets, Traps, Hooks, Lines, and Miscellaneous Gears categories listed in the International Standard Statistical Classification of Fishing Gears (ISSCFG, 2016). They use more than one type of gear for catching each fish species that land at the fishing harbour. But the fishermen use only trawl nets for catching the cephalod molluscan, Sepia pharaonis. In general, fishing is carried out year-long but a seasonal trend is evident in using different gears. Fishing is banned from mid-April to mid-May on the operation of mechanized crafts to conserve fishery resources. The traditional crafts using gillnets, seines, and the lines carryout fishing activities throughout the year; however, the fishermen abstain from fishing activity at sea during cyclone warning times as advised by the India Meteorological Department, Government of India. All twenty marine species harvested from the coastal waters of Visakhapatnam occur at different depths which range from 0 to 2,870 m. The fin fish, Xiphias gladius is the largest in size at maturity and it is the only species that occurs commonly throughout the year whereas all other species vary in size at maturity from seven to $250 \mathrm{~cm}$ and occur for three to ten months for harvest. Of the twenty species, Lutjanus argentimaculatus, Johnius dussumieri, Trichurus lepturus, Katsuwonus pelamis, and Xiphias gladius spawn throughout the year while all other species spawn seasonally.

Morphometric analysis of marine fishery resources of Visakhapatnam coast: Truman (1999) reported that morphological characters are important in fisheries biology to measure discreteness and relationships among various taxonomic categories. He also stated that phenotypic plasticity in fishes is important to adapt to environmental changes by way of modification of their physiology and behaviour. In effect, the fishes display changes in their morphology, reproduction, or survival in order to mitigate the effects of environmental variation. Rutherford et al. (1987) stated that behavioural and physiological characters of fishes respond quickly to local conditions and fluctuate greatly during an individual's life-span. Crowl and Covich (1990) mentioned that phenotype of individuals of fishes usually does not 
respond to fluctuating environmental conditions and hence phenotype characters are stable and easily quantifiable. Different authors reported that the length-weight relationships are important among biometric relationships as useful tools in fish biology (Pauly, 1993; King 1995; Petrakis and Stergiou, 1995; Santos et al., 2002; Ferreira et al., 2008). Phenotypic features of fishes such as total length, standard length, head length, depth of the body and weight are important for use as a valid method to identify the specimens of fish collected with their systematic morphology (Karunanidhi et al., 2017). In the present study, keeping the importance of phenotypic features of fishes in view, these features have been measured for all the twenty edible marine species landing at Visakhapatnam fishing harbour. The standard length or total length of these fishes mostly not tallied with the size values of these fishes at their maturity given by Marine Products Exports Development Authority, Kochi, India, and hence, necessitates further study in detail in different pockets of coastal waters along the entire coastline of Andhra Pradesh. Additionally, the morphological characters of three Pomfret species, Pampus argenteus, Pampus chinensis, and Parastromateus niger are also described in this study because they have been chosen for the assessment of protein content and heavy metal concentrations in their muscle and gill portions. Nevertheless, the morphometric data provided for these species from Visakhapatnam coastal water forms the basis for further studies and is useful to compare with the same species captured at different locations of coastline of not only Andhra Pradesh but also of other coastal states of India. The information resulting from such studies is expected to be useful for evaluating the length-weight relationships and their value in understanding stock composition, growth, life span, production, and mortality (Stergiou and Moutopoulos, 2001).

Migration types, feeding habit and export value of marine fishery resources of Visakhapatnam coast: Tsukamoto et al. (2009) described different types of migrations in fishes. They are potamodromy, oceanodromy, and diadromy; the last type includes catadromy, anadromy, and amphidromy. Potamodromy involves fishes that move and complete their life cycle entirely in fresh water (Bemis and Kynard, 1997). Oceanodromy involves fishes that move and complete their life cycle entirely in sea water (Teo et al., 2007). Diadromy involves fishes that move between freshwater and marine environments at regular periods in their life cycle (McDowall, 1997). In this migration type, the sub-type, catadromy involves fishes that move from fresh water to sea water for spawning. The sub-type anadromy involves fishes that move sea water to fresh water for spawning while the last sub-type amphidromy involves fishes that move freshwater/estuaries to sea water as larvae and return to fresh water to grow into adults and spawn. In each of these forms of diadromy, different fish species use either freshwater or marine environments for parts of their life histories such as spawning, larval and juvenile growth and maturation (McDowall, 1997; Bradbury et al., 2009). In the present study, among the twenty fish species landing at Visakhapatnam fishing harbour, Stolephorus commersonii is anadromous, Lates calcarifer is catadromous, Himantura bleekeri, Trichiurus lepturus, Pampus chinensis, Parastromateus niger, and Muraenesox talabonoides are amphidromous, and all other species are oceanodromous indicating that most of the fish species sold locally are strictly oceanic species and do not require freshwater habitat at any stage of their life history and hence there is a huge potential for their continued availability which substantiates the Least Concern status given to most of these species by IUCN. Interestingly, all twenty marine species in this study are carnivores, the feeding habit of which enables them to utilize a wide variety of food items and produce their populations abundantly. 
Among the twenty marine species captured from Visakhapatnam coastal waters, several species have been reported to be of export value but only some fin fish species Trichiurus lepturus, Katsuwonus pelamis, Thunnus albacares, Makaira indica, Xiphias gladius, and Rastrelliger kanagurta, and the molluscan, Sepia pharaonis are exported by different companies regularly through local trade center. Of these, $T$. lepturus, K. pelamis, and $X$. gladius have the Least Concern status, $M$. indica and S. pharaonis Data Deficient status, $R$. kanagurta Not Evaluated status and T. albacares Near Threatened status according to IUCN Red list. The species with Data Deficient and Not Evaluated status need to be assessed to know their present status to allow or disallow exports and to regulate their catch. The species with Near Threatened status is the main cause of concern as its continued exploitation for local consumption and exports may lead to its extinction in course of time and hence its catch is to be regulated while taking appropriate measures for its conservation, production, and management. Further, the export and local consumption of species with Least Concern status also need to be regulated because their continued exploitation may lead to a change in their status according to the criteria of IUCN.

Physico-chemical characteristics of sea water at Visakhapatnam: Satynarayana et al. (1992) reported on the seasonal variations in physico-chemical parameters in surface harbour waters and coastal waters of Visakhapatnam. The study provided annual averages of $\mathrm{pH}$, salinity, temperature, dissolved oxygen, and biological oxygen demand which indicated that there is an increase in $\mathrm{pH}$ and salinity from harbour to coastal waters; the lower $\mathrm{pH}$ at harbour is attributed to the drainage of acidic effluents from the nearby industries while the lower salinity at harbour is attributed to the influx of industrial effluents and domestic sewage, and the resultant dilution. Temperature is relatively high in summer and low in winter. The surface water in harbour is super-saturated with dissolved oxygen due to intense photosynthetic activity by several planktonic blooms as reported previously by Ganapati and Raman (1979). The coastal water showed lower values of dissolved oxygen which is attributed to offshore divergence as reported previously by Satyanarayana et al. (1987). Biological oxygen demand is more in the harbour and far less in coastal waters; the high BOD in the harbour is because of moderate pollution. In the present study, the values of $\mathrm{pH}$, salinity, dissolved oxygen, biological oxygen demand and temperature recorded in May from the coastal surface waters $500 \mathrm{~m}$ away from the Visakhapatnam harbour almost agree with the values of the same parameters reported for the coastal surface waters of Visakhapatnam by Satynarayana et al. (1992). Since the harbour area of Visakhapatnam is consistently polluted due to influx of various industrial effluents and domestic sewage, it is felt that the values of $\mathrm{pH}$, salinity, temperature, dissolved, oxygen and biological oxygen demand would further go up far beyond the values of the same parameters for harbour surface water reported by Satynarayana et al. (1992). Similarly, Raman (1995) also reported all time high values of dissolved oxygen in harbour surface waters and attributed these values to the periodic outbursts of phytoplanktons, especially Skeletonema costatum which use nutrients from industrial and urban wastes. Further, he also stated that pollution-tolerant species such as Capitella capitata inhabited the bottom sediments of the harbour that contained a heavy load of organic matter. The increased pollution levels led to the disappearance of stenoecious species which live only in a restricted range of habitats, and they are replaced by other pollution-tolerant species. Therefore, the study suggests that it is imperative to regulate the influx of industrial effluents and domestic sewage into the harbour waters to enable the latter to recover back to its previous state with their native stenoecious species and to maintain the water chemistry of coastal surface waters for the sustainability of marine fishery resources. 
Protein content in Pampus argenteus, $P$. chinensis and Parastromateus niger collected from coastal waters of Visakhapatnam: Hajeb et al. (2009) reported that fish is a healthy food in contrast to meat, poultry, and eggs for most of the people in the world, especially in developing countries. It provides comparatively cheap and readily available protein sources in addition to long chains of n-3 fatty acids, amino acids and vitamins, and minerals. Sikorski (1990) stated that among all the fishes, marine fish are very important sources of protein and different mineral components. Priatni et al. (2018) analyzed total protein content in nine marine fish species, Leiognathus equulus, Mystacoleucus padangensis, Nemipterus hexodon, Oxyleotris marmorata, Selaroides leptolepis, Terapon jarbua, and Trichiurus lepturus; the protein content in these fish species varied from 65 to $86 \%$ weight by weight. These authors also reported that total protein content in Pampus argenteus was $61.07 \%$ weight by weight. In the present study, it is found that the total protein content is only $18.28 \%$ in $P$. argenteus (w/w) indicating that its protein content is far less when compared to the total protein content reported for the same species by Priatni et al. (2018); this huge variation could be attributable to the size, age, and food sources available to the species in the habitat where individuals of this species live. Further, the total protein content is also at low level in $P$. chinensis and P. niger, it is $16.24 \%(\mathrm{w} / \mathrm{w})$ in the former and $19.58 \%(\mathrm{w} / \mathrm{w})$ in the latter. Therefore, the study reveals that the three Pomfret species now analyzed are not very important protein sources. However, further studies on the total protein content in individuals of these Pomfret species with different age and size collected from different locations of coastal waters of Visakhapatnam are required to confirm the findings of the present study.

Heavy metal pollution in gills and muscle of Pampus argenteus, $P$. chinensis, and Parastromateus niger collected from coastal waters of Visakhapatnam: Vinodhini and Narayanan (2009) reported that fish gill is an important site for the entry of heavy metals and in fact, it is the first target organ for exposure to heavy metals in coastal water. The concentration of metals in the gill is an indicator of the level of the metals in the water where the fish live while the concentration in liver and kidney represents storage of metals (Romea et al., 1999; Rao and Padmaja, 2000). Since the gill in fishes is the first organ that gets exposed to coastal water, it is usually recommended as an environmental indicator organ of water pollution than any other organs of fishes (Obasohan et al., 2008; Yilmaz, 2009).

In fishes, heavy metal bioaccumulation is species-dependent. Bio-accumulation of heavy metals is related to the bioconcentration capacity of species, their habitats, and feeding habits such as carnivore, herbivore, and omnivore. Further, variations of concentrations of heavy metals in different species is also related to body weight and length, gender, age, and growing rate, body portions analyzed, and physiological conditions. The type and level of water pollution, chemical form of metal in the water, water temperature, $\mathrm{pH}$, dissolved oxygen concentration, water transparency are other factors that influence heavy metal concentrations in different fishes (Al-Majed and Preston, 2000; Canli and Atli, 2003; Yilmaz, 2005; Agoes and Hamami, 2007; Fariba et al., 2009; Raja et al., 2009). In puffer fishes of the Gulf of Mannar Marine Biosphere Reserve, South India, Takifugu oblongus, Lagocephalus guentheri, Arothron hispidus, Chelodan patoca, and Arothron immaculatus the high accumulation of heavy metals is attributed to their carnivorous feeding nature and bottom habitat (Karunanidhi et al., 2017). Water temperature plays an important role in the rate of uptake and elimination of heavy metals and causes differences in metal deposition rates in different organs affecting certain physiological processes. Further, regulatory ability, behaviour and feeding habits play a main role in the accumulation differences in different organs (Nwabunike, 2016). 
Arsenic is a naturally available element in soils, water, and living organisms. An acute high-level exposure to arsenic causes vomiting, diarrhea, anemia, liver damage, and death. Long term exposure could cause skin disease, hypertension, some forms of diabetes, and cancer (Centeno et al., 2005). Most arsenic in our diet is present in organic form (WHO, 2011). Most arsenic in marine organisms is in non-toxic organic form and does not constitute a risk for human health (Maher, 1983). In the present study, the detected concentration of arsenic in both muscle and gill portions individually or combined in all the three Pomfret species, Pampus argenteus, $P$. chinensis, and Parastromateus niger is highly negligible and far below the recommended limit fixed by FSSAI (2011) and hence is safe for consumption by humans. Set standard limit for arsenic is not fixed in the regulations of EU (2006), FAO/WHO (2011) and MAFF and is not possible to evaluate the detected concentration of arsenic in the three Pomfret species. Nevertheless, the arsenic concentration in these fish species is negligible and totally safe for consumption.

The heavy metals, cadmium, mercury, and lead are non-essential and have no known essential role in living organisms but exhibit extreme toxicity even at very low metal exposure levels and pose great threats to all forms of life especially human health (Eisler, 1985; Jarup, 2003). Toxic effects occur when excretory, metabolic, storage, and detoxification mechanisms are not able to counter the uptake of metals (Obasohan et al., 2008) and the effects result in physiological and histopathological changes (Oliveira Ribeiro et al., 2005; Rajamanickam and Muthuswamy, 2008; Vinodhini and Narayanan 2009; Georgieva et al., 2014). Ingestion of any significant amount of cadmium causes immediate poisoning and damage to liver and kidneys (El-Moshelhy et al., 2014). Cadmium is highly toxic and shows nephrotoxic effects and longterm exposure to this heavy metal causes bone damage (Chaitanya et al., 2017). Lead causes long-term harm in adults in terms of increasing risk of high blood pressure, kidney damage, and neurological problems. In young children, exposure to lead causes toxic effects and they suffer from profound and permanent adverse health effects, particularly related to development of brain and nervous system (Voegborlo et al., 2012). In the present study, the detected concentrations of cadmium and lead in the muscle and gills of the Pomfret species do not fall within the recommended limits set by all the regulating agencies. Cadmium concentration detected in the muscle and gills of $P$. argenteus, in the gills of $P$. chinensis, and $P$. niger is within the permitted limit and in the muscle of $P$. chinensis and $P$. niger slightly exceeded the permitted limit according to EU (2006) and FAO (2003) regulations. But the detected cadmium concentration in both muscle, and gills of all the three fish species is beyond the recommended limit according to MAFF and FSSAI (2011). Mercury concentration in the muscle of $P$. argenteus and in the muscle and gills of $P$. niger is within the recommended limit according to EU (2006), FAO/WHO (2011), FAO (2003), MAFF and FSSAI (2011) while its concentration in the gills of $P$. argenteus, muscle and gills of $P$. chinensis exceeded slightly beyond the permitted limit by all these regulating agencies. Lead concentration detected in the muscle and gills of $P$. argenteus, $P$. chinensis, and in the muscle of $P$. niger is within the permitted limit according to the regulations of EU (2006), FAO/WHO (2011), FAO (2003), MAFF and FSSAI (2011). But the lead concentration levels in the gills of $P$. niger slightly exceeded the permitted limit according to EU (2006), FAO/WHO (2011), and FSSAI (2011) prominently exceeded the permitted limit according to FAO (2003) and MAFF. The results of the study on the bioaccumulation of heavy metals in the muscle and gill parts of Pomfret species indicate that consuming these fish species from coastal waters of Visakhapatnam is largely not harmful because the levels of heavy metals analyzed are either below or slightly beyond the permissible limits. However, it is important to state that cadmium levels in the muscle of $P$. chinensis and $P$. niger, and mercury levels in the muscle of $P$. chinensis are 
causes of concern because their muscle portion is the main edible part. The people who consume $P$. chinensis and $P$. niger regularly or frequently are more prone to the health risks associated with high levels of cadmium, and mercury. In general, the bioaccumulation levels of all four heavy metals detected in all the three Pomfret species are either within or slightly in excess of the recommended limits standardized by different national and international agencies. Such low levels of these heavy metals particularly in the muscle portion of these fish species could be attributable to their carnivorous feeding habit and age factor; the finding of this study is in agreement with Khalid (2004) who reported that carnivorous fish accumulate lower levels of heavy metals than herbivorous fish and also with Abdallah (2008) who reported higher metal concentrations in omnivorous or herbivorous fish than carnivorous fish. Further, the higher cadmium levels in the muscle of $P$. chinensis and $P$. niger, and higher mercury levels in the muscle of $P$. chinensis could also be attributable to their migratory nature as these two fish species are amphidromous which requires them to move between freshwater/estuaries and sea water as part of their life cycle during which they get exposed to polluted aquatic environment which is usually associated with freshwater/estuarine areas. The low mercury levels in the muscle of $P$. niger could be attributable to the less mercury pollution in the aquatic environment, especially in freshwater/estuarine areas. P. argenteus is an oceanodromous species and migrates within the ocean for feeding and spawning due to which it is not very prone to polluted aquatic environment which is usually the case with freshwater/estuarine areas which are characteristically the reception sites for industrial effluents and domestic sewages/wastes. As a result, $P$. argenteus carries out its life cycle relatively in pollution-free aquatic environment while the other two pomfret species get exposed to polluted aquatic environment, especially in freshwater/estuarine areas. Nevertheless, $P$. chinensis and $P$. niger could be used to evaluate the health condition of the aquatic environment and use them as biomarkers of heavy metal contamination in coastal waters of Visakhapatnam.

The study suggests strategy elements for the conservation and management of marine fishery resources of coastal waters of Andhra Pradesh State. Location-based studies are required to document marine fishery resources, their migration types, feeding habits, their present status according to IUCN criteria and risk of their exposure to marine pollution during their life cycle. Further, studies on heavy metal contamination in each edible marine fish species are required to alert fish consumers and take appropriate actions to control marine pollution. Finally, regular monitoring of marine resources is essential to improve the quality of sea-food against contaminants, especially heavy metals to protect the health of fish consumers and for managing the coastal waters in an ecologically sustainable manner.

\section{CONCLUSIONS}

The State of Andhra Pradesh has a long coastline which is spread over nine coastal districts. This coastline area is the home for a diversity of marine fishery resources comprising of several groups of fishes, crustaceans, molluscs, and other marine organisms. An inventory of marine fishery resources of this state indicated that a total of 212 species consisting of fin fishes, crustaceans, and molluscs which have food value. Among fin fishes, 26 species belong to Elasmobranch group, 146 species to Teleosts group. The crustaceans are represented by 30 species while molluscs are represented by 10 species. Fin fishes and crustaceans are the major contributors to fishery sector. 
At Fishing Harbour and local markets of Visakhapatnam, a total of twenty edible marine species are commonly sold. They are Himantura bleekeri, Lutjanus argentimaculatus, Johnius dussumieri, Tachysurus thalassinus, Stolephorus commersonii, Trichiurus lepturus, Lates calcarifer, Rastrelliger kanagurta, Scomberomorus commerson, S. guttatus, Katsuwonus pelamis, Thunnus albacares, Coryphaena hippurus, Makaira indica, Xiphias gladius, Pampus argenteus, P. chinensis, Parastromateus niger, Muraenesox talabonoides, and Sepia pharaonis. According to IUCN red list, S. commerson and T. albacares belong to Near Threatened category; S. guttatus, M. indica, and S. pharaonis to Data Deficient category; $H$. bleekeri, $T$. thalassinus, $R$. kanagurta, $P$. argenteus, $P$. chinensis, and $M$. talabonoides to Not Evaluated category; and all other species belong to Least Concern category. Of these, $S$. commersonii is anadromous, L. calcarifer is catadromous, H. bleekeri, T. lepturus, $P$. chinensis, $P$. niger, and $M$. talabonoides are amphidromous, and all other species are oceanodromous indicating that most of the fish species sold locally are strictly oceanic species and do not require freshwater habitat at any stage of their life history and hence there is a huge potential for their continued availability which substantiates the Least Concern status given to most of these species by IUCN. Interestingly, all twenty marine species in this study are carnivores, the feeding habit of which enables them to utilize a wide variety of food items and produce their populations abundantly. However, the populations of these species are consistently subjected to degradation and fragmentation of their habitats due to unorganized and organized human activities involving urbanization, industrialization, tourism. Further, these species are highly subjected to over-exploitation and over-consumption of these fishery resources locally and for making money through seafood exports through the Visakhapatnam Sea Food Export Trade Center (VSFETC). Keeping this situation in view, it is suggested that there is an urgent need to evaluate the present status of the edible marine species of Visakhapatnam coastal waters in order to take proper measures for their conservation, management and utilization as food and revenue sources.

The fishermen of the coastline of Visakhapatnam use Surrounding nets, Seine nets, Trawls, Lift nets, Gillnets and Entangling nets, Traps, Hooks and Lines, and Miscellaneous Gears categories listed in the International Standard Statistical Classification of Fishing Gears (ISSCFG 2016) to harvest marine fishery resources. In general, fishing is carried out year-long but, a seasonal trend is evident in using different gears. Fishing is banned from mid-April to mid-May on the operation of mechanized crafts to conserve fishery resources. The traditional crafts using gillnets, seines, and the lines carryout fishing activities throughout the year; however, the fishermen abstain from fishing activity at sea during cyclone warning times. All twenty marine species harvested from the coastal waters of Visakhapatnam occur at different depths ranging from 0 to $2,870 \mathrm{~m}$. The fin fish, Xiphias gladius is the largest in size at maturity and it is the only species that occurs commonly throughout the year whereas all other species vary in size at maturity from seven to $250 \mathrm{~cm}$ and occur for three to ten months for harvest. Of the twenty species, Lutjanus argentimaculatus, Johnius dussumieri, Trichurus lepturus, Katsuwonus pelamis, and Xiphias gladius spawn throughout the year while all other species spawn seasonally.

Morphometric data were provided for all twenty marine fish species collected from the coastal waters of Visakhapatnam for future use to evaluate the length-weight relationships and their value in understanding stock composition, growth, life span, production, and mortality. Additionally, the morphological characters of three Pomfret species, Pampus argenteus, Pampus chinensis, and Parastromateus niger are described because they have been chosen for the assessment of protein content and heavy metal concentrations of arsenic, cadmium, mercury, and lead in their muscle and gill portions. 
The physico-chemical parameters such as $\mathrm{pH}$, salinity, dissolved oxygen, biological oxygen demand, and temperature were recorded in summer season from the coastal surface waters $500 \mathrm{~m}$ away from the Visakhapatnam harbour. The study indicated that $\mathrm{pH}$ and salinity values are high, while the values of other parameters are low; this trend is attributed to offshore divergence which dilutes pollution levels. It is found that the harbour area of Visakhapatnam is consistently polluted due to influx of various industrial effluents and domestic sewage; for this reason, the study of these parameters is not needed but it underlined the need to regulate pollutants into the harbour waters to enable the latter to recover back to its previous state with their native stenoecious species and maintain the water chemistry of coastal surface waters for the sustainability of marine fishery resources.

The total protein content in the muscle of Pomfret species, Pampus argenteus, Pampus chinensis, and Parastromateus niger was analyzed; it was $18.28 \%$ in the first species, $16.24 \%$ in the second species and $19.58 \%$ in the last species. These values have been attributed to the size, age, and food sources available to these species in their habitat. Further studies on the total protein content in individuals of these Pomfret species with different age and size collected from different locations of coastal waters of Visakhapatnam are required to evaluate accurately the potential of these fishes as protein sources depending on their feeding environment.

In fishes, heavy metal bioaccumulation is species-dependent and is related to the bioconcentration capacity of individual species, their habitats, and feeding habits such as carnivore, herbivore, and omnivore. Further, variations of concentrations of heavy metals in different species is also related to body weight and length, gender, age, growing rate, body portions analyzed, and physiological conditions. The type and level of water pollution, chemical form of metal in the water, water temperature, $\mathrm{pH}$ value, dissolved oxygen concentration, water transparency are other factors that influence heavy metal concentrations in different fishes. Water temperature plays an important role in the rate of uptake and elimination of heavy metals and causes differences in metal deposition rates in different organs affecting certain physiological processes. In this study, the detected concentration of arsenic in both muscle and gill portions individually or combined in all the three Pomfret species, Pampus argenteus, $P$. chinensis, and Parastromateus niger is highly negligible and far below the recommended limit fixed by FSSAI (2011) and hence is safe for consumption by humans. The detected concentrations of cadmium and lead in the muscle and gills of the three Pomfret species do not fall within the recommended limits set by all the regulating agencies. Cadmium concentration detected in the muscle and gills of $P$. argenteus, in the gills of $P$. chinensis and $P$. niger is within the permitted limit and in the muscle of $P$. chinensis and $P$. niger slightly exceeded the permitted limit according to EU (2006) and FAO (2003) regulations. But the detected cadmium concentration in both muscle and gills of all the three fish species is beyond the recommended limit according to MAFF and FSSAI (2011). Mercury concentration in the muscle of $P$. argenteus and in the muscle and gills of $P$. niger is within the recommended limit according to EU (2006), FAO/WHO (2011), FAO (2003), MAFF, and FSSAI (2011) while its concentration in the gills of $P$. argenteus, muscle and gills of $P$. chinensis exceeded slightly beyond the permitted limit by all these regulating agencies. Lead concentration detected in the muscle and gills of $P$. argenteus, $P$. chinensis, and in the muscle of $P$. niger is within the permitted limit according to the regulations of EU (2006), FAO/WHO (2011), FAO (2003), MAFF, and FSSAI (2011). But the lead concentration levels in the gills of $P$. niger slightly exceeded the permitted limit according to EU (2006), FAO/WHO (2011), and FSSAI (2011) 
prominently exceeded the permitted limit according to FAO (2003) and MAFF. Therefore, the results indicate that consuming these fish species from coastal waters of Visakhapatnam is largely not harmful because the levels of heavy metals analyzed are either below or slightly beyond the permissible limits. However, it is important to state that cadmium levels in the muscle of $P$. chinensis and $P$. niger, and mercury levels in the muscle of $P$. chinensis are causes of concern in the long run because their muscle portion is the main edible part. The people who consume $P$. chinensis and $P$. niger regularly or frequently are more prone to the health risks associated with high levels of cadmium and mercury.

The study suggests that strategies are needed for the conservation and management of marine fishery resources of coastal waters of Andhra Pradesh State. Location-based studies are required to document marine fishery resources, their migration types, feeding habits, their present status according to IUCN criteria, and risk of their exposure to marine pollution during their life cycle. Further, studies on heavy metal contamination in each edible marine fish species are required to alert fish consumers and take appropriate actions to control marine pollution. Finally, regular monitoring of marine resources is essential improve the quality of seafood against contaminants, especially heavy metals to protect the health of fish consumers and for managing the coastal waters in an ecologically sustainable manner.

\section{ACKNOWLEDGEMENTS}

We thank the Andhra University, Visakhapatnam, for providing physical facilities to carry out this research work. We also thank Dr. K. Venkata Ramana and Dr. Ch. Prasada Rao, Department of Botany, Andhra University, for help in the collection of fish species. 


\section{REFERENCES}

1. Abdallah M. A. M., 2008 - Trace element levels in some commercially valuable fish species from coastal waters of Mediterranean Sea, Egypt, Journal of Marine Systems, 73, 114-122.

2. Agoes S. and Hamami, 2007 - Trace metal concentrations in shrimp and fish collected from Gresik coastal waters, Indonesia, Science Asia, 33, 235-238.

3. Akköz C., 2016 - The determination of some pollution parameters, water quality and heavy metal concentrations of Aci Lake (Karapinar/Konya, Turkey), Transylvanian Review of Systematical and Ecological Research, 18.3, 1-20.

4. Al-Majed N. and Preston M., 2000 - An assessment of the total and methyl mercury content of zooplankton and fish tissue collected from Kuwait territorial waters, Marine Pollution Bulletin, 40, 298-307.

5. Amundsen P. A., Staldvik F. J., Lukin A. A., Kashulin N. A., Popova O. A. and Reshetnikov Y. S., 1997 - Heavy metal contamination in freshwater fish from the border region between Norway and Russia, Science of the Total Environment, 201, 211-224.

6. Astratinei V. and Varduca I., 2008 - Effect of metal pollution on aquatic microorganisms: a case study in mining areas (Romania), Transylvanian Review of Systematical and Ecological Research, 6, 109-116.

7. Atlindag A. and Yigit S., 2005 - Assessment of heavy metal concentrations in the food web of lake Beysehir, Turkey, Chemosphere, 60, 552-556.

8. Aziz T. N. A. and Hashim N. R., 2011 - Heavy metal concentrations in an important mangrove palm (Nypa fruticans), in Rembau-Linggi Mangrove Forest (Peninsular Malaysia), Transylvanian Review of Systematical and Ecological Research, 12, 111-116

9. Bemis W. E. and Kynard B., 1997 - Sturgeon rivers: an introduction to acipenseriform biogeography and life history, Environmental Biology of Fishes, 48, 167-183.

10. Bradbury I. R., Coulson M. W., Campana S. E., Baggs E. and Bentzen P., 2009 - Postglacial recolonization and the loss of anadromy in rainbow smelt from coastal Newfoundland, in Haro A. J., Smith K. L., Rulifson R. A., Moffitt C. M., Klauda R. J., Dadswell M. J., Cunjak R. A., Cooper J. E., Beal K. L. and Avery T. S. (eds), 79-96, Challenges for diadromous fishes in a dynamic global environment, American Fisheries Society Symposium 69, Bethesda, Maryland.

11. Burton P. J., Balisky A., Coward L. P., Cumming S. G. and Kneeshaw D. D. 1992 - The value of managing for biodiversity, The Forestry Chronicle, 68, 225-237.

12. Canli M. and Atli G., 2003 - The relationships between heavy metal (Cd, Cr, Cu, Fe, Pb, Zn) levels and the size of six Mediterranean fish species, Environmental Pollution, 121, 129-136.

13. Centeno J. A., Gray M. A., Mullick J. G., Tchounwou P. B. and Tseng C., 2005 - Arsenic in drinking water and health issues. Treatments and effects in ecology and human health, Resolution Press, Christ Church, 415-436.

14. Chaitanya I., Gayathri C. and Byragireddy T., 2017 - Heavy metal accumulation in commercial fish species of Bheemili, Visakhapatnam, Andhra Pradesh, India, International Journal of Applied Research, 3, 320-324.

15. Crowl T. A. and Covich A. P., 1990 - Predator-induced life-history shifts in a freshwater snail, Science, 247, 949-951.

16. Curtean-Bănăduc A., Burcea A., Mihuţ C.-M., Berg V., Lyche J. L. and Bănăduc D., 2020 Bioaccumulation of persistent organic pollutants in the gonads of Barbus barbus (Linnaeus, 1758), Ecotoxicology and Environmental Safety, 201, 110852, 10, https://doi.org/10.1186/s12302-020-00348-z.

17. Dalman O., Demirak A. and Balci A., 2006 - Determination of heavy metals (Cd, Pb) and trace elements $(\mathrm{Cu}, \mathrm{Zn})$ in sediments and fish of the Southeastern Aegean Sea (Turkey) by atomic absorption spectrometry, Food Chemistry, 95, 157-162. 
18. Donati E. R., 2018 - Heavy metals in the environment: microorganisms and bioremediation, CRC Press, Boca Raton, FL, USA, 332.

19. Dural M., Goksu M. Z. L. and Ozak A. A., 2007 - Investigation of heavy metal levels in economically important fish species captured from Tuzla Lagoon, Food Chemistry, 102, 415421.

20. Eisler R., 1985 - Cadmium hazard to fish, wildlife and invertebrates: a synoptic review, United States Fish and Wildlife Service Biological Report, 85, 1-30.

21. El-Moshelhy K. M., Othman A. I., El-Azem H. A. and El-Metwally M. E. A., 2014 Bioaccumulation of heavy metals in some tissues of fish in the Red Sea, Egypt, Egyptian Journal of Basic and Applied Sciences, 30, 1-9.

22. EU 2006 - European Commission of Regulation (EC) No. 1881/2005 of 19th December 2006, Setting maximum levels for certain contaminants in foodstuffs, L 364/5, 20.12.2006.

23. FAO 2003 - The State of World Fisheries and Aquaculture, FAO, Rome, Italy, 176.

24. FAO/WHO 2011 - Food Standards Programme Codex Committee on Contaminants in Foods, Fifth Session, The Hague, The Netherlands, 21-25 March 2011, http:www.fao.org/tempref/codex/Meetings/CCCF/CCCF5/cf05_INF.pdf.

25. Fariba Z., Hossein T., Siamak A. R., Meshkini A. A. and Mohammad R., 2009 - Determination of copper, zinc and iron levels in edible muscle of three commercial fish species from Iranian coastal waters of the caspian Sea, Journal of Animal and Veterinary Advances, 8, 1288-2009.

26. Ferreira S., Sousa R., Delgado J., Carvalho D. and Chada T., 2008 - Weight-length relationships for demersal fish species caught off the Madeira archipelago (eastern-central Atlantic), Journal of Applied Ichthyology, 24, 93-95.

27. FSSAI 2011 - Food Safety and Standards Authority of India (Contaminants, Toxins and Residues) Regulations, 2011, Ministry of Health and Welfare, Government of India, New Delhi.

28. Gado M. S. and Midany S. A., 2003 - Studies on some heavy metals pollutants in cultured Oreochromis niloticus fish at kafer El-Sheikh Governorate, Kafer El-Sheikh, Journal of Veterinary Medicine, 1, 83-85.

29. Ganapati P. N. and Raman A. V., 1979 - Organic pollution and Skeletonema blooms in Visakhapatnam harbor, Current Science, 8, 184-187.

30. Georgieva E., Velcheya I., Yancheya V. and Stoyanova S., 2014 - Trace metal effects on gill epithelium of common carp Cyprinus carpio L. (Cyprinidae), Acta Zoologica Bulgarica, 66, 277 282.

31. Goutte A., Cherel Y., Churlaud C., Ponthus J. P., Masse G. and Bustamante P., 2015 - Trace elements in Antarctic fish species and the influence of foraging habitats and dietary habits on mercury levels, Science of the Total Environment, 538, 743-749.

32. Hajeb P., Jinap S., Ismail A., Fatimah A. B., Jamilah B. and Abdul Rahim M., 2009 - Mercury level in commonly consumed marine fishes in Malaysia, Food Control, 20, 79-84.

33. Huang X., Qin D., Gao L., Hao Q., Chen Z., Wang P., Tang S., Wu S., Jiangb H. and Qiu W., 2019 - Distribution, contents and health risk assessment of heavy metal(loid)s in fish from different water bodies in Northeast China, RSC Advances, 9, 33130-33139.

34. Immanuel S. and Syda Rao G., 2012 - Social status of hook and line fishermen in Visakhapatnam, Fishery Technology, 49, 204-209.

35. ISSCFG 2016 - International Standard Statistical Classification of Fishing Gear. The relationship between the current ISSCFG codes and those used in the previous classification (1980), Handbook of Fishery Statistics, Food and Agriculture Organization of the United Nations, Rome.

36. Jarup L., 2003 - Hazards of heavy metal contamination, British Medical Bulletin, 68, 167-182. 
37. Jayaram K. C., 1999 - The freshwater fishes of the Indian regions, Narendra Publishing House, Delhi, 551.

38. Khoshnood Z. and Khoshnood R., 2013 - Health risks evaluation of heavy metals in sea food, Transylvanian Review of Systematical and Ecological Research, 15.1, 47-80.

39. Kar, D., 2007 - Fundamentals of limnology and aquaculture biotechnology, Daya Publishing House, New Delhi, 609.

40. Kar D., Sur P., Mandal S. K., Saha T. and Kole R. J., 2008 - Assessment of heavy metal pollution in surface water, International Journal of Environmental Science and Technology, 5, 119-124.

41. Kar D., 2013 - Wetlands and lakes of the World, Springer, London, 687.

42. Kar D., 2019 - Wetlands diversity and their fishes in Assam, India, Transylvanian Review of Systematical and Ecological Research, 21.3, 47-80.

43. Karunanidhi K., Rajendran R., Pandurangan D. and Arumugam G., 2017 - First report on distribution of heavy metals and proximate analysis in marine edible puffer fishes collected from Gulf of Mannar Marine Biosphere Reserve, South India, Toxicology Reports, 4, 319-327.

44. Khalid A., 2004 - Seasonal determination of soil heavy metals on muscles tissues of Siganus revaltus and Sargus sargus fish from El-mex bay and Eastern harbor, Alexandra, Egypt, Egyptian Journal Aquatic Biology and Fisheries, 8, 65-81.

45. King M., 1995 - Fisheries biology, assessment and management, Fishing News Books, Oxford, England, 107-111.

46. MAFF 2000 - Monitoring and surveillance of non-radioactive contaminants in the aquatic environment and activities regulating the disposal of wastes at sea, 1997, Aquatic Environment Monitoring Report No. 53, Center for Environment, Fisheries and Aquaculture Science, Lowestoft, UK.

47. Maher W. A., 1983 - Inorganic arsenic in marine organisms, Marine Pollution Bulletin, 14, 308310.

48. McDowall R. M., 1997 - The occurrence and distribution of diadromy among fishes, in Dadswell M. J., Klauda R. J., Moffitt C. M., Saunders R. L., Rulifson R. A. and Cooper J. E. (eds), 1-13, American Fisheries Society, Symposium 1, Bethesda, Maryland.

49. Mehouel F., Bouayad L., Hammoudi A. H., Ayadi O. and Regad F., 2019 - Evaluation of the heavy metals (mercury, lead, and cadmium) contamination of sardine (Sardina pilchardus) and swordfish (Xiphias gladius) fished in three Algerian coasts, Veterinary World, 12, 7-11.

50. Mensoor M. and Said A., 2018 - Determination of heavy metals in freshwater fishes of the Tigris River in Baghdad, Fishes, 3, 23.

51. Miller J. R., Lechler P. J., Hudson-Edwards K. A. and Macklin M. G., 2002 - Lead isotopic fingerprinting of heavy metal contamination, Rio Pilcomayo Basin, Bolivia, Geochemistry, Exploration, Environment, Analysis, 2, 225-233.

52. Muddula Krishna N., Govinda Rao V. and Venu D., 2016 - Taxonomic diversity, species composition, distribution, conservation and abundance of rocky shore intertidal fishes in the Visakhapatnam, East Coast of India, Journal of Experimental Zoology India, 19, 277-283.

53. Nadal M. M., Schuhmacher M. and Domingo J. L., 2004 - Metal pollution of soils and vegetation in an area with petrochemical industry, Science of the Total Environment, 321, 59-69.

54. Nikinmaa M., 2014 - An Introduction to Aquatic Toxicology. Academic Press, Cambridge, MA, USA, 252.

55. Nwabunike M. O., 2016 - The effects of bioaccumulation of heavy metals on fish fin over two years, Journal of Fisheries Livestock Production, 4, 170. 
56. Obasohan E. E., Oronsaye J. A. O. and Eguavoen O. I., 2008 - A comparative assessment of the heavy metal loads in the tissues of a common catfish (Clarias gariepinus) from Ikpoba and Ogba Rivers in Benin City Nigera, African Science, 9, 13-23.

57. Oliveira Ribeiro C. A., Vollaire Y., Sanchez-Chardi A. and Roche H., 2005 - Bioaccumulation and the effects of organochlorine pesticides PAH and heavy metals in the eel (Anguilla anguilla) at the Camargue Nature Reserve, France, Aquatic Toxicology, 74, 53-69.

58. Papagiannis I., Kagalou I., Leonardos J., Petridis D. and Kalfakakou V., 2004 - Copper and zinc in four freshwater fish species from Lake Pamyotis (Greece), Environment International, 30, 357-362.

59. Pauly D., 1993 - Fish byte section editorial, 16, Naga, ICLARM, Quart, ICLARM, Naga, Philippines 16, 26.

60. Pauley D., Christensen V., Dalsgaard J., Froese R. and Torres Jr. F., 1998 - Fishing down marine food webs, Science News, 279, 860-86.

61. Petrakis G. and Stergiou K. I., 1995 - Weight-length relationships for 33 fish species in Greek waters, Fisheries Research, 21, 465-469.

62. Priatni S., Ratnaningrum D., Kosasih W., Sriendah E., Srikandace Y., Rosmalina T. and Pudjiraharti S., 2018 - Protein and fatty acid profile of marine fishes from Java Sea, Indonesia, Biodiversitas, 19, 1737-1742.

63. Raja P., Veerasingam S., Suresh G., Marichamy G. and Venkatachalapathy R., 2009 - Heavy metals concentration in four commercially valuable marine edible fish species from Parangipettai Coast, South East Coast of India, International of Journal of Animal and Veterinary Advances 1, 10-14.

64. Rajamanickam V. and Muthuswamy N., 2008 - Effect of heavy metals on the level of vitamin, total lipid and glycogen reserves in the liver of common carp (Cyprinus carpio L.), Maejo International Journal of Science and Technology, 2, 95-100.

65. Rajeshkumar S., Liu Y., Zhang X., Ravikumar B., Bai G. and Li X., 2018 - Studies on seasonal pollution of heavy metals in water, sediment, fish and oyster from the Meiliang Bay of Taihu Lake in China, Chemosphere, 191, 626-638.

66. Raman A.V., 1995 - Pollution effects in Visakhapatnam harbour, India: An overview of 23 years of investigations and monitoring, Helgolander Meeresuntersuchungen, 49, 633-645.

67. Rao L. M. and Padmaja G., 2000 - Bioaccumulation of heavy metals in M cyprinoids from the harbor waters of Visakhapatnam, Bulletin of Pure Applied Science, 19, 77-85.

68. Romoea M., Siaub Y., Sidoumou Z. and Gnassia-Barelli M., 1999 - Heavy metals distribution in different fish from the Mauritiana coast, Science of the Total Environment, 232, 169-175.

69. Rutherford D. A., Echelle A. A. and Maughan O. E., 1987 - Changes in the fauna of the Little River drainage, south-eastern Oklahoma, 1948-1955 to 1981-1982: a test of the hypothesis of environmental degradation, in Community and evolutionary ecology of North American stream fishes, Matthews W. J. and Heins D. C. (eds), 178-183, University of Oklahoma Press, Norman.

70. Santos M. N., Gaspar M. B., Vasconcelos P. and Monteiro C. C., 2002 - Weight-length relationships for 50 fish species of the algarve coast (southern Portugal), Fisheries Research, 59, 289-295.

71. Satyanarayana D., Prasada Reddy B. R., Dileep Kumar M. and Ramesh A., 1987 - Chemical oceanographic studies on the Bay of Bengal north of Visakhapatnam, Contributions in Marine Science, 329-338.

72. Satyanarayana D., Sahu S. D. and Panigraphy P. K., 1992 - Physico-chemical characteristics in the coastal environment of Visakhapatnam - a case study, Journal of the Marine Biological Association of India, 34, 103-109. 
73. Iepure S. and Selescu L., 2009 - Relationship between heavy metals and hyporheic invertebrate community structure in the middle basin of the Arieş River (Transylvania, Romania), Transylvanian Review of Systematical and Ecological Research, 7, 125-148.

74. Sikorski Z. E., 1990 - Resources nutritional composition and preservation, CRC Press, Boca Raton, Florida, USA.

75. Sreekrishna Y., 2002 - Traditional fishing craft and gear in India, Advances in Harvest Technology, ICAR Winter School Manual, CIFT, Cochin, 101-139.

76. Stergiou K. I. and Moutopoulos D. K., 2001 - A review of length-weight relationships of fishes from Greek marine waters, Naga, ICLARM Quarterly, 24, 24-39.

77. Syda Rao G., Prathibha Rohit G. M. and Rajkumar U., 2008 - Marine fisheries of Andhra Pradesh: An Appraisal, Marine Fisheries Information Science T\&E Series, 196, 1-17.

78. Taiwo I. O., Olopade O. A. and Bamidele N. A., 2019 - Heavy metal concentration in eight fish species from Epe Lagoon (Nigeria), Transylvanian Review of Systematical and Ecological Research, 21.1, 69-83.

79. Teo S. L. H., Boustany A., Dewar H., Stokesbury M. J. W., Weng K. C., Beemr S., Seitz A. C., Farwell C. J., Prince E. D. and Block B. A., 2007 - Annual migrations, diving behavior, and thermal biology of Atlantic bluefin tuna, Thunnus thynnus, on their Gulf of Mexico breeding grounds, Marine Biology, 151, 1-18.

80. Truman C., 1999 - A note on the examination of morphometric differentiation among fish populations: the Truss system, Turkish Journal of Zoology, 23, 259-263.

81. Tsukamoto K., Miller M. J., Kotake A., Aoyama J. and Uchida K., 2009 - The origin of fish migration: the random escapement hypothesis, American Fisheries Society Symposium, 69, 4561.

82. Valset A., Bouchon-Navaro Y., Louis M. and Bouchon C., 2007 - Weight-length relationships for 20 fish species collected in the mangroves of Guadeloupe (Lesser Antilles), Applied Ichthyology, 24, 99-100.

83. Venkataraman K. and Wafer M., 2005 - Coastal and marine biodiversity of India, Indian Journal of Marine Science, 34, 57-75.

84. Vinodhini R. and Narayanan M., 2009 - Heavy metal induced histopathological alterations in selected organs of the Cyprinus carpio L. (Common carp), International Journal of Environmental Research, 3, 95-100.

85. Voegborlo R. B., Atta A. and Agorku E. S., 2012 - Total mercury distribution in different tissues of six species of freshwater fish from the Kpong hydroelectric reservoir in Ghana, Environmental Modeling and Assessment, 184, 3259-3265.

86. Yi Y.-J. and Zhang S.-H., 2012 - Heavy metal (Cd, Cr, Cu, Hg, Pb, Zn) concentrations in seven fish species in relation to fish size and location along the Yangtze River, Environmental Science \& Pollution Research, 19, 3989-3996.

87. Yilmaz A. B., 2003 - Levels of heavy metals (Fe, Cu, Ni, Cr, Pb, and Zn) in tissue of Mugil cephalus and Trachurus mediterraneus from Iskenderun Bay, Turkey, Environmental Research, 92, 277-281.

88. Yilmaz A. B., 2005 - Comparison of heavy metal levels of grey mullet (Mugil cephalus L.) and Sea Bream (Sparus aurata L.) caught in Isekenderun Bay (Turkey), Turkish Journal Veterenary and Animal Science, 29, 257-262.

89. Yilmaz A. B. and Yilmaz L., 2007 - Influences of sex and seasons on levels of heavy metals in tissues of green tiger shrimp (Penaeus semisculcatus de Hann, 1844), Food Chemistry, 101, 1664-1669. 
90. Yilmaz F., 2009 - The comparison of heavy metal concentrations (Cd, Cu, Mn, Pb, and Zn) in tissues of three economically important fish (Anguilla mugilcephalus and Oreochromis niloticus) inhabiting Koycegiz Lake-Mugla (Turkey), Turkish Journal of Science \& Technology, 4, 7-15.

91. Zubcov N., Zubcov E. and Schlenk D., 2008 - The dynamics of metals of fish from Nistru and Prut rivers (Moldova), Transylvanian Review of Systematical and Ecological Research, 6, 51-58. 